\title{
SHORT TIME FULL ASYMPTOTIC EXPANSION OF HYPOELLIPTIC HEAT KERNEL AT THE CUT LOCUS
}

\author{
YUZURU INAHAMA $^{1}$ and SETSUO TANIGUCHI ${ }^{2}$ \\ ${ }^{1}$ Faculty of Mathematics, Kyushu University, Motooka 744, Nishi-ku, Fukuoka, 819-0395, Japan; \\ email: inahama@math.kyushu-u.ac.jp \\ ${ }^{2}$ Faculty of Arts and Science, Kyushu University, Motooka 744, Nishi-ku, \\ Fukuoka, 819-0395, Japan; \\ email: se2otngc@artsci.kyushu-u.ac.jp
}

Received 21 July 2016; accepted 30 May 2017

\begin{abstract}
In this paper we prove a short time asymptotic expansion of a hypoelliptic heat kernel on a Euclidean space and a compact manifold. We study the 'cut locus' case, namely, the case where energyminimizing paths which join the two points under consideration form not a finite set, but a compact manifold. Under mild assumptions we obtain an asymptotic expansion of the heat kernel up to any order. Our approach is probabilistic and the heat kernel is regarded as the density of the law of a hypoelliptic diffusion process, which is realized as a unique solution of the corresponding stochastic differential equation. Our main tools are S. Watanabe's distributional Malliavin calculus and T. Lyons' rough path theory.
\end{abstract}

2010 Mathematics Subject Classification: 60H07 (primary); 58J65, 35K08, 41A60 (secondary)

\section{Introduction}

Short time asymptotics of heat kernels is a huge topic in analysis, probability and geometry and have been studied intensively and extensively. In this paper we focus on the short time off-diagonal asymptotic expansion of heat kernels. We do not discuss the on-diagonal asymptotics, the estimates or the logarithmic asymptotics of heat kernels. Even if we restrict our attention to the off-diagonal expansion, so many papers have been published and our references are probably far from complete.

Known proofs of off-diagonal asymptotics are either analytic or probabilistic and our proof is the latter. The first probabilistic proof was given by

(c) The Author(s) 2017. This is an Open Access article, distributed under the terms of the Creative Commons Attribution licence (http://creativecommons.org/licenses/by/4.0/), which permits unrestricted re-use, distribution, and reproduction in any medium, provided the original work is properly cited. 
Molchanov [38] in the case of the standard heat kernel on a Riemannian manifold. In it pinned diffusion processes are used. Bismut [8] was first to use Malliavin calculus, then followed by many others. The Feynman-Kac representation is the key in these probabilistic approaches.

On a Riemannian manifold $\mathcal{N}$, if $x$ and $x^{\prime}$ are outside the cut locus, the following asymptotic expansion of the standard heat kernel is well known:

$$
p_{t}\left(x, x^{\prime}\right) \sim e^{-d\left(x, x^{\prime}\right)^{2} / 2 t} t^{-n / 2}\left(c_{0}+c_{1} t+c_{2} t^{2}+\cdots\right) \quad \text { as } t \searrow 0 .
$$

Here, $n=\operatorname{dim} \mathcal{N}$ and $d\left(x, x^{\prime}\right)$ is the Riemannian distance between $x, x^{\prime} \in \mathcal{N}$. Ben Arous [7] proved that the heat kernel of a hypoelliptic diffusion process of a wide class on a Euclidean space admits essentially the same expansion as above if $x$ and $x^{\prime}$ are outside the cut locus. In this case, however, the distance should be replaced by the sub-Riemannian distance.

When the two points are not near, this asymptotics becomes quite difficult. Molchanov [38] already mentioned that there is an example of a Riemannian manifold $\mathcal{N}$ and $x, x^{\prime} \in \mathcal{N}$ in the cut locus such that the factor $t^{-n / 2}$ in (1.1) must be replaced by $t^{-\left(n+n^{\prime}\right) / 2}$ with some $n^{\prime} \neq 0$. Therefore, the offdiagonal asymptotics in the cut locus case is qualitatively different and much less understood than the case of 'near points.' (Note that we use the term 'cut locus' in a loose sense in this section. What we precisely mean by the term will be given in Assumption (B2) in the next section.)

Our present work aims to study the short time off-diagonal asymptotic expansion for hypoelliptic heat kernels in the cut locus case in a satisfactory general way. It has the following three features. To our knowledge, no other works have all of these features simultaneously:

(a) The manifold and the hypoelliptic diffusion process on it are rather general. In other words, this is not a study of special examples.

(b) The case is allowed where the set of energy-minimizing paths (or controls) which connect the two points under consideration is assumed to be a compact manifold of finite positive dimension. In particular, this lies outside the usual 'non-cut-locus' hypothesis.

(c) The asymptotic expansion is full, that is, the polynomial part of the asymptotics is up to any order.

A recent paper by Barilari et al. [3] satisfy (a) and (b), but only the leading term of the asymptotic expansion is obtained. Chang and Li [12] satisfies (b) and (c) for special examples. (Many papers obtain the leading term in the 'cut locus' case for special examples. See [4-6, 10, 17, 43, 49, 50] among many others.) Note that 
the result in [7] is formulated on a Euclidean space, but can be modified to the manifold case (if the manifold and the diffusion process are not too wild). Hence, it satisfies (a) and (c).

On a Euclidean space, however, there are two famous works which satisfy (b), (c) and the latter half of (a). Both of them are probabilistic and use generalized versions of Malliavin calculus. One is Takanobu and Watanabe [47]. They use Watanabe's distributional Malliavin calculus developed in [51, 52]. The other is Kusuoka and Stroock [32]. They use their version of generalized Malliavin calculus developed in [31]. In this paper we use the former.

Though we basically follow Takanobu-Watanabe's argument, the main difference is that we use Lyons' rough path theory together, which is something like a deterministic version of the SDE theory. The main advantage of using rough path theory is that while the usual Ito map that is the solution map of an SDE is discontinuous, the Lyons-Itô map that is the solution map of a rough differential equation (RDE) is continuous.

This fact enables us to do 'local analysis' of the Lyons-Itô map (for instance, restricting the map on a neighborhood of its critical point and doing Taylor-like expansion) in a somewhat similar way we do in the Fréchet calculus. Recall that in the standard SDE theory, this type of local operation is often very hard and sometimes impossible, due to the discontinuity of the Itô map. For this reason, the localization procedure in [47] looks so complicated that it might be difficult to generalize their method if rough path theory did not exist.

In this paper we first reprove and generalize the main result in [47] in the Euclidean setting by using rough path theory. Malliavin calculus for Brownian rough path was already studied in [25, 27] in the proof of large deviation principle (LDP for short) for conditional measures. It turned out that these two theories fit very well.

The strategy of our proof is as follows: first, the LDP above implies that contributions from the complement of the set of energy minimizers (action minimizers) are negligibly small. In other words, only a neighborhood of the set of energy minimizers matters. Next, around each energy minimizer in the Cameron-Martin space, we take a small neighborhood with respect to the rough path topology with certain nice properties. Since the set of energy minimizers is compact, we need to compute contributions from finitely many of them only. Then, on each of such small neighborhoods, we do local analysis. To prove the asymptotic expansion up to any order, we use a modified version of Watanabe's asymptotic theory given in [47], which can be regarded as a 'localized' version of his asymptotic theory developed in $[22,52]$.

Even in this Euclidean setting many parts of the proof are technically improved thanks to rough path theory. We believe that the following are worth mentioning: 
(i) Large deviation upper bound (Theorem 3.8 and Lemma 7.1). (ii) Asymptotic partition of unity (Section 6). (iii) A Taylor-like expansion of the Lyons-Itô map (Section 3.3) and the uniform exponential integrability lemma for the ordinary and the remainder terms of the expansion (Lemmas 5.2 and 5.3). (iv) Quasisure analysis for solutions of SDEs, although this is implicit in this paper. (See [25, 27] for details.)

Then, we study the manifold case. Recall that Malliavin calculus for a manifoldvalued SDE was studied by Taniguchi [48]. Thanks to rough path theory and the localized version of Watanabe's asymptotic theory, it is enough to localized in a small neighborhood of energy minimizers in the geometric rough path space. Restricted on such a small neighborhood, the (Lyons-)Itô map corresponding to the given SDE on the manifold can easily be transferred to one on a linear space. For these reasons, without much technical effort the problem reduces to the one on a Euclidean space. As a result, the proof of the asymptotic expansion in the manifold setting is not so different from the one in the Euclidean setting.

Of course, there is a possibility that our main result can be proved without rough path theory, but we believe that the theory is quite suitable for this problem and gives us a very clear view (in particular, in the manifold case).

The structure of the paper is as follows: in Section 2, we provide setting, assumptions and our main results. First, we discuss the Euclidean case. Our assumptions are weaker that those in [47] and hence our main result (Theorem 2.2) in the Euclidean case generalizes the main result in [47]. Next, we formulate a parallel problem on a compact manifold. As mentioned before, our main result (Theorem 2.5) in the manifold case satisfies all of (a), (b) and (c) above. At the end of the section, a few examples are given.

Sections 3-7 are devoted to proving our main theorem in the Euclidean setting (Theorem 2.2). In Section 3, we gather various facts from relevant research areas such as Malliavin calculus, rough path theory and differential geometry. All of them are already known basically, but some of them are not elementary. They will be used in the proof of the asymptotic expansion.

In Section 4, we calculate the skeleton ODE driven by an energy-minimizing Cameron-Martin path and show that the solution satisfies a naturally defined Hamiltonian ODE. We look at this well-known argument from a viewpoint of rough path theory. In Section 5, we prove the uniform exponential integrability of Wiener functionals which appear in the proof of the asymptotic expansion. Since we use rough path theory, the Taylor-like expansion of the Lyons-Itô map is deterministic and the remainder terms satisfy a simple and reasonable estimate. This simplifies our proof.

In Section 6, we study an asymptotic partition of unity which was first introduced in [47]. Though it plays an important role in the proof of the 
asymptotics in [47], it is of very complicated form. We construct a rough path version of it, which is written in terms of Besov norms of Brownian rough path and looks simple and natural.

Section 7 is the main part of our proof of Theorem 2.2. Our main tool is a modified version of Watanabe's asymptotic theorem. In Section 8, we prove our main result in the manifold case (Theorem 2.5).

\section{Setting, assumptions and main results}

2.1. Setting: the Euclidean case. Let $\mathcal{W}=C_{0}\left([0,1], \mathbb{R}^{r}\right)$ be the set of the continuous functions from $[0,1]$ to $\mathbb{R}^{r}$ which start at 0 . This is equipped with the usual sup-norm. The Wiener measure on $\mathcal{W}$ is denoted by $\mu$. We denote by

$$
\mathcal{H}=\left\{h \in \mathcal{W} \mid \text { absolutely continuous and }\|h\|_{\mathcal{H}}^{2}:=\int_{0}^{1}\left|h_{s}^{\prime}\right|^{2} d s<\infty\right\}
$$

the Cameron-Martin subspace of $\mathcal{W}$. In some (nonprobabilistic) literatures, the derivative of Cameron-Martin paths are called controls. The triple $(\mathcal{W}, \mathcal{H}, \mu)$ is called the classical Wiener space. The canonical realization on $\mathcal{W}$ of $r$-dimensional Brownian motion is denoted by $\left(w_{t}\right)_{0 \leqslant t \leqslant 1}=\left(w_{t}^{1}, \ldots, w_{t}^{r}\right)_{0 \leqslant t \leqslant 1}$.

Let $V_{i}: \mathbb{R}^{d} \rightarrow \mathbb{R}^{d}$ be a vector field with sufficient regularity $(0 \leqslant i \leqslant r)$. Precise conditions on $V_{i}$ will be specified later. For a small parameter $\varepsilon \in(0,1]$, we consider the following SDE of Stratonovich type:

$$
d X_{t}^{\varepsilon}=\varepsilon \sum_{i=1}^{r} V_{i}\left(X_{t}^{\varepsilon}\right) \circ d w_{t}^{i}+\varepsilon^{2} V_{0}\left(X_{t}^{\varepsilon}\right) d t \quad \text { with } X_{0}^{\varepsilon}=x \in \mathbb{R}^{d} .
$$

When necessary, we write $X_{t}^{\varepsilon}=X^{\varepsilon}(t, x, w)$ or $X^{\varepsilon}(t, x)$ and sometimes write $\lambda_{t}^{\varepsilon}=\varepsilon^{2} t$. When $\varepsilon=1$, we simply write $X_{t}=X_{t}^{1}$. By the scaling property of Brownian motion, the laws of the processes $\left(X_{t}^{\varepsilon}\right)_{t \geqslant 0}$ and $\left(X_{\varepsilon^{2} t}\right)_{t \geqslant 0}$ are the same.

Now we introduce the skeleton ODE which corresponds to SDE (2.1). For a Cameron-Martin path $h \in \mathcal{H}$, we consider the following controlled ODE:

$$
d \phi_{t}=\sum_{i=1}^{r} V_{i}\left(\phi_{t}\right) d h_{t}^{i} \quad \text { with } \phi_{0}=x \in \mathbb{R}^{d} .
$$

The solution will often be denoted by $\phi_{t}(h), \phi(t, x, h)$, and so forth. Note the absence of the drift term in (2.2).

Let $\mathcal{V}$ be an $n$-dimensional linear subspace of $\mathbb{R}^{d}(1 \leqslant n \leqslant d)$ and $\Pi_{\mathcal{V}}: \mathbb{R}^{d}$ $\rightarrow \mathcal{V}$ be the orthogonal projection. (For our purpose, we may and sometimes will assume without loss of generality that $\mathcal{V}=\mathbb{R}^{n} \times\left\{\mathbf{0}_{d-n}\right\}$, where $\mathbf{0}_{d-n}$ is the zero 
vector of $\mathbb{R}^{d-n}$.) Set $Y_{t}^{\varepsilon}=\Pi_{\mathcal{V}}\left(X_{t}^{\varepsilon}\right)$ and $\psi_{t}=\Pi_{\mathcal{V}}\left(\phi_{t}\right)$, which will often be denoted by $Y^{\varepsilon}(t, x, w)$, and $\psi(t, x, h)$, respectively.

For $a \in \mathcal{V}$, define $\mathcal{K}_{a}=\{h \in \mathcal{H} \mid \psi(1, x, h)=a\}$. We set $d_{a}:=\min \left\{\|h\|_{\mathcal{H}} \mid\right.$ $\left.h \in \mathcal{K}_{a}\right\}$ and $\mathcal{K}_{a}^{\min }=\left\{h \in \mathcal{K}_{a} \mid\|h\|_{\mathcal{H}}=d_{a}\right\}$. It is known that if $\mathcal{K}_{a} \neq \emptyset$, then $\inf \left\{\|h\|_{\mathcal{H}} \mid h \in \mathcal{K}_{a}\right\}$ actually attains a minimum and $\mathcal{K}_{a}^{\min } \neq \emptyset$. (We can see this from the goodness of the rate function in a Schilder-type large deviation principle on the rough path space.) We basically assume $x \notin \Pi_{\mathcal{V}}^{-1}(a)$ and $\mathcal{K}_{a} \neq \emptyset$, which imply $0<d_{a}<\infty$.

2.2. Assumptions: the Euclidean case. In this subsection we introduce assumptions. First, we impose two conditions on the coefficient vector fields. The first one is on regularity of $V_{i}$.

(A1): $V_{i}: \mathbb{R}^{d} \rightarrow \mathbb{R}^{d}$ is of $C^{\infty}$ with bounded derivatives of all order $\geqslant 1(0 \leqslant i$ $\leqslant r)$.

Note that $V_{i}$ itself may have linear growth. When $V_{i}$ is also bounded, $V_{i}$ is said to be of $C_{b}^{\infty}$. ( $C_{b}^{k}$ is similarly defined for $1 \leqslant k<\infty$.) This assumption is very standard in Malliavin calculus and most of the results in Malliavin calculus for SDEs are proved under this assumption. Under (A1), a global solution $X^{\varepsilon}$ of (2.1) uniquely exists and $X_{t}^{\varepsilon}$ is smooth in the sense of Malliavin calculus for any $(t, \varepsilon) \in[0,1]^{2}$ and their Sobolev norm of any index is bounded in $(t, \varepsilon) \in[0,1]^{2}$. Similarly, (A1) is a standard assumption for the skeleton ODE (2.2). A unique global solution $\phi$ for any $h \in \mathcal{H}$ exists and, moreover, $\phi(\cdot, x, h)$ is Fréchet smooth in $h \in \mathcal{H}$ for any $x$ under (A1).

Next we introduce a kind of Hörmander's bracket generating condition not only on the 'upper' space $\mathbb{R}^{d}$, but also on the 'lower' space $\mathcal{V}$. We denote by $\sigma\left[Y_{1}^{\varepsilon}\right]$ and $\sigma\left[\psi_{1}\right]$ the Malliavin covariance matrix of $Y_{1}^{\varepsilon}$ and the deterministic Malliavin covariance matrix of $\psi_{1}$, respectively. (Precise definitions will be given in Section 3.)

Set

$$
\Sigma_{1}=\left\{V_{i} \mid 1 \leqslant i \leqslant r\right\} \quad \text { and } \quad \Sigma_{k}=\left\{\left[V_{i}, W\right] \mid 0 \leqslant i \leqslant r, W \in \Sigma_{k-1}\right\}
$$

for $k \geqslant 2$ recursively. Note that the drift vector field is not involved in the definition of $\Sigma_{1}$.

(A2): We say that (A2) holds if either of the following two conditions holds:

(i) At the starting point $x \in \mathbb{R}^{d}, \bigcup_{k=1}^{\infty}\left\{W(x) \mid W \in \Sigma_{k}\right\}$ spans $\mathbb{R}^{d}$ in the sense of linear algebra. 
(ii) There exists $L \in \mathbb{N}$ such that

$$
\inf _{x \in \mathbb{R}^{d}} \inf _{\eta \in \mathcal{V},|\eta|=1} \sum_{k=1}^{L} \sum_{W \in \Sigma_{k}}\left\langle\Pi_{\mathcal{V}} W(x), \eta\right\rangle^{2}>0 .
$$

The first one is quite standard. The latter one is called the uniform partial Hörmander condition. This condition (A2) implies that $Y_{t}^{\varepsilon}=Y^{\varepsilon}(t, x, w)$ is nondegenerate in the sense of Malliavin calculus for any $t>0$ and $\varepsilon \in(0,1]$. Moreover, there exist positive constants $v$ and $C_{p}(1<p<\infty)$ such that

$$
\left\|\operatorname{det} \sigma\left[Y_{1}^{\varepsilon}\right]^{-1}\right\|_{L^{p}} \leqslant C_{p} \varepsilon^{-v} \quad(1<p<\infty, 0<\varepsilon \leqslant 1) .
$$

(See Kusuoka and Stroock [30, Theorem 2.17 and Lemma 5.1].) Note that $v$ is independent of $p$. Non-degeneracy implies that, if $\varepsilon>0$ and $t>0, \delta_{a}\left(Y_{t}^{\varepsilon}\right)$ is well defined as a positive Watanabe distribution (that is generalized Wiener functional), where $\delta_{a}$ stands for the delta function at $a \in \mathcal{V}$, and its generalized expectation $\mathbb{E}\left[\delta_{a}\left(Y_{t}^{\varepsilon}\right)\right]$ equals the smooth density of the law of $Y_{t}^{\varepsilon}$ with respect to the Lebesgue measure on $\mathcal{V}$ for every $a$. (By the way, the partial Hörmander condition only at the starting point does not imply the non-degeneracy of $Y_{t}^{\varepsilon}$. There is a simple counterexample.)

From here we introduce assumptions on the subset $\mathcal{K}_{a}^{\min }$ of energy minimizers $\left(a \neq \Pi_{\mathcal{V}} x\right)$.

(B1): Assume that $\mathcal{K}_{a} \neq \emptyset$ and the deterministic Malliavin covariance matrix $\sigma\left[\psi_{1}\right](h)$ is non-degenerate for any $h \in \mathcal{K}_{a}^{\min }$.

Note that non-degeneracy of $\sigma\left[\psi_{1}\right](h)$ is equivalent to surjectivity of the tangent map $D \psi_{1}(h): \mathcal{H} \rightarrow \mathcal{V}$. If we use terminology of sub-Riemannian geometry, this condition loosely means that any element of $\mathcal{K}_{a}^{\text {min }}$ is a nonabnormal geodesics. (Keep in mind that we are not in the framework of sub-Riemannian geometry, however.)

As we wrote, when $\mathcal{K}_{a}^{\min }$ is not a finite set things usually get quite complicated. To exclude the cases where $\mathcal{K}_{a}^{\min }$ is not nice, we assume the following condition:

(B2): $\mathcal{K}_{a}^{\min }$ is a smooth, compact manifold of finite dimension $n^{\prime}$ regularly embedded in $\mathcal{H}$.

In the above condition the case $n^{\prime}=0$ is also allowed, which means that $\mathcal{K}_{a}^{\min }$ is a finite set. Note that compactness of $\mathcal{K}_{a}^{\min }$ is essential and will be used throughout this paper. It will turn out in Remark 4.5 that (A1) and (B1) imply compactness of $\mathcal{K}_{a}^{\min }$ with respect to $\mathcal{H}$-topology. From this viewpoint, the compactness assumption in (B2) seems natural. 
Finally, we assume positivity of the Hessian of $\left.I\right|_{\mathcal{K}_{a}}$ on $\mathcal{K}_{a}^{\text {min }}$, where $I(h):=$ $\|h\|_{\mathcal{H}}^{2} / 2$, in an appropriate sense. Without the positivity our proof breaks down. Since $\left.I\right|_{\mathcal{K}_{a}}$ attains minimum on $\mathcal{K}_{a}^{\text {min }}$, its Hessian is naturally defined on $\mathcal{K}_{a}^{\text {min }}$ without assuming additional structures. The precise definition of the Hessian at $h \in \mathcal{K}_{a}^{\min }$ is as follows: for any $k \in T_{h} \mathcal{K}_{a}$ (which is equivalent to $k \in \operatorname{ker} D \psi_{1}(h)$ ), take a smooth curve $\left(-\tau_{0}, \tau_{0}\right) \ni \tau \mapsto c(\tau) \in \mathcal{K}_{a}$ such that $c(0)=h$ and $c^{\prime}(0)=k$. (Here, $\tau_{0}>0$. Because of (A1) and the implicit function theorem, $\mathcal{H}$-neighborhood of $h$ in $\mathcal{K}_{a}$ has a manifold structure. Hence, the notion of 'smooth' curve in the neighborhood is well defined.) Then, we set

$$
I^{\prime \prime}(h)\langle k, k\rangle:=\left.\frac{d^{2}}{d \tau^{2}}\right|_{\tau=0} I(c(\tau)) .
$$

As is well known, this is independent of the choice of the curve $c$ and $I^{\prime \prime}(h)$ becomes a bounded bilinear form on $T_{h} \mathcal{K}_{a} \times T_{h} \mathcal{K}_{a}$. It is obvious that $I^{\prime \prime}(h)\langle k, k\rangle=0$ if $k \in T_{h} \mathcal{K}_{a}^{\min }$.

We assume that the elements of $T_{h} \mathcal{K}_{a}^{\min }$ are the 'only directions' in $T_{h} \mathcal{K}_{a}$ such that $I^{\prime \prime}(h)$ vanishes. For simplicity, we set $\mathcal{H}_{0}(h):=T_{h} \mathcal{K}_{a} \cap\left(T_{h} \mathcal{K}_{a}^{\min }\right)^{\perp}$, which is a closed subspace of codimension $n+n^{\prime}$.

(B3): For any $h \in \mathcal{K}_{a}^{\min }$ and any $k \in \mathcal{H}_{0}(h) \backslash\{0\}, I^{\prime \prime}(h)\langle k, k\rangle>0$.

Some equivalent conditions to (B3) are known. (See for example Bismut [8] or Takanobu and Watanabe [47].) One obvious example is that (B3) remains the same if we replace ' $k \in \mathcal{H}_{0}(h) \backslash\{0\}$ ' by ' $k \in T_{h} \mathcal{K}_{a} \backslash T_{h} \mathcal{K}_{a}^{\text {min }}$ '. Note also that (B3) is also equivalent to the exponential integrability of a certain quadratic Wiener functional that appears in the proof of our main theorem. (See Section 5.)

Another possible choice of sufficient condition in place of (B3) is the 'nonfocality condition.' In [14, Section 3], equivalence of (B3) and the nonfocality condition is shown under slightly different assumptions from ours. Although (B3) is quite typical and does not look bad, some people may prefer the nonfocality condition since it is written in terms of finite-dimensional mathematics, in particular, Hamiltonian ODEs. However, we do not use the nonfocality condition in this paper.

We introduce a family $G^{\varepsilon}(w)=G(\varepsilon, w)$ of scalar-valued $\mathbb{D}_{\infty}$-functionals $(0<\varepsilon \leqslant 1)$. Main examples we have in mind are multiplicative functionals that appear in the Feynman-Kac(-Itô) formulas. Here, $\mathbb{D}_{\infty}$ stands for the space of test Wiener functionals.

(C1): for any $\varepsilon \in(0,1], G^{\varepsilon}$ is a real-valued smooth Wiener functional, that is, $G^{\varepsilon} \in \mathbb{D}_{\infty}$ and the following asymptotic expansion holds for any $h \in \mathcal{H}$ :

$$
G\left(\varepsilon, w+\frac{h}{\varepsilon}\right) \sim \Gamma_{0}(h)+\varepsilon \Gamma_{1}(w ; h)+\varepsilon^{2} \Gamma_{2}(w ; h)+\cdots \quad \text { in } \mathbb{D}_{\infty} \text { as } \varepsilon \searrow 0
$$


uniformly on $\left\{h \in \mathcal{H} \mid\|h\|_{\mathcal{H}} \leqslant \rho\right\}$ for any $\rho>0$. Here, $\Gamma_{0}(h)$ is a smooth function on $\mathcal{H}$ and $\Gamma_{j}(\cdot ; h) \in \mathbb{D}_{\infty}$ depends smoothly on $h \in \mathcal{H}(j \geqslant 1)$.

In the asymptotic expansion of heat kernels, the odd order terms of $\varepsilon$ vanish. (As usual we use a scale change $t=\varepsilon^{2}$.) To formulate and prove this phenomenon, we set the following assumption:

(C2): For any $h \in \mathcal{K}_{a}^{\min }, \Gamma_{j}(\cdot ; h) \in \mathbb{D}_{\infty}$ is an even (respectively odd) Wiener functional in $w$-argument if $j$ is even (respectively odd).

We say a (generalized) Wiener functional $F$ is even (respectively odd) if $F(-w)=F(w)$ (respectively $F(-w)=-F(w)$ ). These are well defined since the map $w \mapsto-w$ leaves $\mu$ invariant. If $G^{\varepsilon} \equiv 1$, then (C2) is clearly satisfied.

REMARK 2.1. The reader may wonder why we introduce the projection $\Pi_{\mathcal{V}}$ in our formulation. In the early days of Malliavin calculus, this type of assumption was common in the study of 'partially hypoelliptic' problems (See $[30,48]$ and references within.) This formulation probably has its origin in Eells-Elworthy's construction of Brownian motion on a Riemannian manifold (Example 2.8). Recently, this kind of formulation attracted attention from mathematical finance, too. (See [14] for instance.)

2.3. Main result: the Euclidean case. In this subsection we present our main result in the Euclidean setting. The proof of this theorem will be given in Sections 6-7. An explicit expression of the leading term $c_{0}$ will be given in (7.17). If $\Gamma_{0}$ is non-negative and not identically zero on $\mathcal{K}_{a}^{\text {min }}$, then $c_{0}>0$. Note that the left hand side of the asymptotic expansion (2.5) below is the generalized expectation.

THEOREM 2.2. Let $x \in \mathbb{R}^{d}$ and $a \in \mathcal{V}$ such that $\Pi_{\mathcal{V}}(x) \neq a$. Assume (A1), (A2), (B1), (B2), (B3) and (C1). Then, we have the following asymptotic expansion:

$$
\mathbb{E}\left[G(\varepsilon, w) \delta_{a}\left(Y_{1}^{\varepsilon}\right)\right] \sim e^{-d_{a}^{2} / 2 \varepsilon^{2}} \varepsilon^{-\left(n+n^{\prime}\right)}\left(c_{0}+c_{1} \varepsilon+c_{2} \varepsilon^{2}+\cdots\right) \text { as } \varepsilon \searrow 0
$$

for certain constants $c_{j} \in \mathbb{R}(j \geqslant 0)$. If we assume $(\mathbf{C 2})$ in addition, then $c_{2 j+1}=$ $0(j \geqslant 0)$.

REMARK 2.3. The only difference between our Theorem 2.2 and [47, Theorem 5.1] is the Hörmander's bracket generating condition (A2). In [47] the stronger version of bracket generating condition is assumed at every $x \in \mathbb{R}^{d}$ on the upper space. (By 'stronger' we mean the drift vector field $V_{0}$ is not involved in the condition.) 
On the other hand, we assume the weaker version of bracket generating condition. Moreover, the condition is either 'on the lower space' or 'at the starting point only on the upper space' as in (A2). Therefore, we believe we made sizable relaxation of the bracket generating condition.

Put in a broader context, however, whether this improvement is large or not is somewhat unclear. In other words, we do not know how many of the newly allowed examples by this relaxation satisfy the other assumptions. (For instance, under the weaker version of Hörmander's condition, (B1) may not hold very often.) It needs further investigations.

On the other hand, the relaxation of the Hörmander-type condition to the 'partial' version (that is the bracket generating condition on the lower space) is crucial for our geometric purpose. Without it we could not even treat the case of Brownian motion on a Riemannian manifold. (See Remark 2.1 and Examples 2.8-2.12.)

The formulation of Theorem 2.2, (in particular, the generalized expectation) may look too general for nonexperts of Watanabe's distributional Malliavin calculus. Therefore, we give basic examples below.

EXAMPLE 2.4. Consider the case $\mathcal{V}=\mathbb{R}^{d}$ and write $a=x^{\prime}(\neq x)$. As usual we set $t=\varepsilon^{2}$. Then, the heat kernel $p_{t}\left(x, x^{\prime}\right)$ associated with the differential operator $L:=(1 / 2) \sum_{i=1}^{r} V_{i}^{2}+V_{0}$ is expressed as

$$
p_{\varepsilon^{2}}\left(x, x^{\prime}\right)=\mathbb{E}\left[\delta_{x^{\prime}}\left(X^{\varepsilon}(1, x, w)\right)\right] .
$$

In this example $G(\varepsilon, w) \equiv 1$. (In our convention, $\left(e^{t L} f\right)(x)=\int_{\mathbb{R}^{d}} p_{t}\left(x, x^{\prime}\right)$ $f\left(x^{\prime}\right) d x^{\prime}$, where $e^{t L}$ denotes the heat semigroup associated with $L$.)

Let $C$ be a tempered smooth function on $\mathbb{R}^{d}$ which is bounded from below. Then, the heat kernel $p_{t}^{C}\left(x, x^{\prime}\right)$ associated with $L^{C}:=(1 / 2) \sum_{i=1}^{r} V_{i}^{2}+V_{0}-C$ is expressed as

$$
p_{\varepsilon^{2}}^{C}\left(x, x^{\prime}\right)=\mathbb{E}\left[\exp \left(-\varepsilon^{2} \int_{0}^{1} C\left(X^{\varepsilon}(s, x, w)\right) d s\right) \delta_{x^{\prime}}\left(X^{\varepsilon}(1, x, w)\right)\right] .
$$

In this case $G(\varepsilon, w)=\exp \left(-\varepsilon^{2} \int_{0}^{1} C\left(X^{\varepsilon}(s, x, w)\right) d s\right)$ is the Feynman-Kac multiplicative functional and satisfies (C1). See Ikeda and Watanabe [22, pages 414-415].

Due to the Feynman-Kac-Itô formula, the heat kernels of magnetic Schrödinger operators also admit a similar expression (We omit details. See Ikeda's survey [21, Section 6].) 
2.4. The manifold case. Let $\mathcal{M}$ and $\mathcal{N}$ be compact manifolds with dimension $d$ and $n(1 \leqslant n \leqslant d)$, respectively, and let $\Pi: \mathcal{M} \rightarrow \mathcal{N}$ be a smooth submersion. $\mathcal{N}$ is equipped with a smooth volume vol. (A measure on $\mathcal{N}$ is said to be a smooth volume if it is expressed on each coordinate chart as a strictly positive smooth density function times the Lebesgue measure.)

For vector fields $V_{i}(0 \leqslant i \leqslant r)$ on $\mathcal{M}$, we study the following scaled SDE and its corresponding skeleton ODE:

$$
\begin{gathered}
d X_{t}^{\varepsilon}=\varepsilon \sum_{i=1}^{r} V_{i}\left(X_{t}^{\varepsilon}\right) \circ d w_{t}^{i}+\varepsilon^{2} V_{0}\left(X_{t}^{\varepsilon}\right) d t \quad \text { with } X_{0}^{\varepsilon}=x \in \mathcal{M}, \\
d \phi_{t}=\sum_{i=1}^{r} V_{i}\left(\phi_{t}\right) d h_{t}^{i} \quad \text { with } \phi_{0}=x \in \mathcal{M} .
\end{gathered}
$$

Set $Y_{t}^{\varepsilon}=\Pi\left(X_{t}^{\varepsilon}\right)$ and $\psi_{t}=\Pi\left(\phi_{t}\right)$. When necessary we write $X_{t}^{\varepsilon}=X^{\varepsilon}(t, x, w)$, and so forth, as before. For $x \in \mathcal{M}$ and $a \in \mathcal{N}$ such that $\Pi(x) \neq a$, we define $\mathcal{K}_{a}, \mathcal{K}_{a}^{\min }$, and $d_{a}$ in the same way as in the Euclidean case.

Malliavin calculus for SDEs on manifolds was studied by Taniguchi [48]. Roughly speaking, most of important results in the flat space case still hold true in the manifold case.

We impose two assumptions on the coefficient vector fields. They are similar to the corresponding ones in the Euclidean case.

(A1)': $V_{i}$ is a smooth vector fields on $\mathcal{M}(0 \leqslant i \leqslant r)$.

Under this condition, $X_{t}^{\varepsilon}$ is smooth in the sense of Malliavin calculus for any $(t, \varepsilon) \in[0,1]^{2}$. (For the definition of manifold-valued smooth Wiener functionals, see $[48]$.

Now we introduce a Hörmander-type condition. $\Sigma_{k}$ is defined as in the Euclidean case. Since the manifold $\mathcal{M}$ is compact, the partial Hörmander condition below is automatically uniform as in (2.3). Therefore, (A2)' and (A2) are parallel.

(A2)': We say that (A2)' holds if either of the following two conditions holds:

(i) At the starting point $x \in \mathcal{M}, \bigcup_{k=1}^{\infty}\left\{W(x) \mid W \in \Sigma_{k}\right\}$ spans $T_{x} \mathcal{M}$ in the sense of linear algebra.

(ii) For every $x \in \mathcal{M}, \bigcup_{k=1}^{\infty}\left\{\left(\Pi_{*}\right)_{x} W(x) \mid W \in \Sigma_{k}\right\}$ spans $T_{\Pi(x)} \mathcal{N}$ in the sense of linear algebra.

Choose a Riemannian metric on $\mathcal{N}$ so that the determinant of the (deterministic) Malliavin covariance of $\mathcal{N}$-valued functionals are well defined. Results in 
Taniguchi [48] or Kusuoka and Stroock [30, Section 5] apply to this case. $Y_{t}^{\varepsilon}$ is non-degenerate in the sense of Malliavin calculus for any $t>0$ and $\varepsilon \in(0,1]$. Moreover, Kusuoka-Stroock's moment estimate (2.4) for $\operatorname{det} \sigma\left[Y_{1}^{\varepsilon}\right]^{-1}$ also holds in this case.

It is known that integration by parts formula also holds for manifold-valued Wiener functional. (See Section 8 for a proof.) Therefore, as in the Euclidean case, if an $\mathcal{N}$-valued smooth Wiener functional $F$ is non-degenerate in the sense of Malliavin, then the composition $T(F)=T \circ F \in \tilde{\mathbb{D}}_{-\infty}$ is well defined as a Watanabe distribution for any distribution $T$ on $\mathcal{N}$. In particular, $\delta_{a}\left(Y_{1}^{\varepsilon}\right)$ a positive Watanabe distribution under (A2)' for every $a \in \mathcal{N}$.

On the other hands, Assumptions (B1), (B2), (B3), (C1) and (C2) need not be modified and will be imposed in the manifold setting again. In this case, too, non-degeneracy of $\sigma\left[\psi_{1}\right](h)$ is equivalent to surjectivity of the tangent map $D \psi_{1}(h): \mathcal{H} \rightarrow T_{a} \mathcal{N}$.

Without loss of generality, we may assume that a Riemannian metric is given on both $\mathcal{M}$ and $\mathcal{N}$. The reason we introduce them is as follows: one on $\mathcal{M}$ is needed when a tubular neighborhood on $\mathcal{M}$ is used. Since Malliavin covariance matrix of $\mathcal{N}$-valued functional is actually a bilinear form on the cotangent space, a metric on the cotangent space is needed when the determinant and the eigenvalues of the Malliavin covariance matrix are considered. For our purpose any Riemannian metric will do. In particular, even if we change the Riemannian metric on $\mathcal{N}$, (A2)' and (B1) (and the other assumptions) remain equivalent. Hence, $\delta_{a}\left(Y_{1}^{\varepsilon}\right)$ does not depend on the choice of the Riemannian metrics on $\mathcal{M}$ and $\mathcal{N}$.

On the other hand, $\delta_{a}$ and $\delta_{a}\left(Y_{1}^{\varepsilon}\right)$ depend on the choice of vol. Since any other smooth volume can be expressed as $\widehat{\operatorname{vol}}(d y)=\rho(y) \operatorname{vol}(d y)$ for some strictly positive smooth function $\rho$ on $\mathcal{N}$, the delta function with respect to $\widehat{\text { vol }}$ is given by $\hat{\delta}_{a}=\rho(a)^{-1} \delta_{a}$. Therefore, it is sufficient to prove Theorem 2.5 below for one particular smooth volume. In the proof of Theorem 2.5 in Section 8, we assume that vol is the Riemannian measure on $\mathcal{N}$.

Theorem 2.5. Let $x \in \mathcal{M}$ and $a \in \mathcal{N}$ such that $\Pi(x) \neq a$. Assume (A1)', (A2)', (B1), (B2), (B3) and (C1). Then, we have the following asymptotic expansion:

$$
\mathbb{E}\left[G(\varepsilon, w) \delta_{a}\left(Y_{1}^{\varepsilon}\right)\right] \sim e^{-d_{a}^{2} / 2 \varepsilon^{2}} \varepsilon^{-\left(n+n^{\prime}\right)}\left(c_{0}+c_{1} \varepsilon+c_{2} \varepsilon^{2}+\cdots\right) \quad \text { as } \varepsilon \searrow 0
$$

for certain constants $c_{j} \in \mathbb{R}(j \geqslant 0)$. If we assume $(\mathbf{C 2})$ in addition, then $c_{2 j+1}=$ $0(j \geqslant 0)$.

REMARK 2.6. Compactness of $\mathcal{M}$ and $\mathcal{N}$ in Theorem 2.5 is assumed for simplicity. Since manifold-valued Malliavin calculus in [48] is developed for a reasonable class of noncompact manifolds, we believe that Theorem 2.5 extends 
to the case of noncompact manifolds under fairly mild assumptions. (In this paper, however, only the compact case is proved. The noncompact case is just a conjecture.)

REMARK 2.7. A remark similar to Example 2.4 holds in the manifold setting, too. In particular, if $\mathcal{M}=\mathcal{N}$ and $\Pi$ is the identity map, then $\mathbb{E}\left[\delta_{x^{\prime}}\left(X^{\varepsilon}(1, x, w)\right)\right]$ is the Feynman-Kac representation of the heat kernel $p_{\varepsilon^{2}}\left(x, x^{\prime}\right)$ associated with $L:=(1 / 2) \sum_{i=1}^{r} V_{i}^{2}+V_{0}$.

However, some important second order differential operators cannot be written in a 'sum of squares' form as in Remark 2.7 above, which is the reason why two manifolds are considered. Here are examples of manifolds $\mathcal{M}$ and $\mathcal{N}$ we have in mind.

The first example is the orthonormal frame bundle over a compact Riemannian manifold. This is a familiar example in stochastic analysis on manifolds and called Eells-Elworthy's construction of Itô's stochastic parallel transport. (See [22, Section V-4] or Hsu [19], Stroock [45] for example.)

EXAMPLE 2.8. Let $\mathcal{N}$ be a compact Riemannian manifold with $\operatorname{dim} \mathcal{N}=n$ and let $\mathcal{M}=\mathcal{O}(\mathcal{N})$ be its orthonormal frame bundle. Hence, $d:=\operatorname{dim} \mathcal{M}=$ $n(n+1) / 2$. Let $\Pi: \mathcal{O}(\mathcal{N}) \rightarrow \mathcal{N}$ be the canonical projection. We take $V_{i}$ to be the $i$ th canonical horizontal vector field on $\mathcal{O}(\mathcal{N})$ for $1 \leqslant i \leqslant n$ and set $V_{0} \equiv 0$.

Let $\hat{a} \in \mathcal{N}$ and consider SDE (2.6) with $r=n, x \in \Pi^{-1}(\hat{a}) \subset \mathcal{O}(\mathcal{N})$ and $a \in \mathcal{N}$. Then, regardless of the choice of $x$, the process $t \mapsto Y^{\varepsilon}(t, x, w)$ is the Brownian motion on $\mathcal{N}$, that is, the diffusion process associated to the (minus one half of) Laplace-Beltrami operator $\triangle_{\mathcal{N}}$ starting at $\hat{a} \in \mathcal{N}$.

In this case (A1)' and (A2)' are clearly satisfied and $\delta_{a}\left(Y^{\varepsilon}(1, x, w)\right)$ is well defined. Moreover, $\mathbb{E}\left[\delta_{a}\left(Y^{\varepsilon}(1, x, w)\right)\right]=p_{\varepsilon^{2}}(\hat{a}, a)$, the heat kernel associated to $-\triangle_{\mathcal{N}} / 2$. Note that the deterministic Malliavin covariance is non-degenerate at any $h \in \mathcal{H}$ since the coefficient vector fields are elliptic at $x$. So, (B1) is also satisfied.

We denote by $\mathcal{H}_{\hat{a}}(\mathcal{N})$ the set of absolutely continuous path $\xi$ on $\mathcal{N}$ starting at $\hat{a}$ with $\mathcal{E}(\xi):=\int_{0}^{1}\left\|\xi_{s}^{\prime}\right\|_{T_{s s} \mathcal{N}}^{2} d s<\infty$. The Cartan development map $h \mapsto \psi(h)$ is a diffeomorphism (of Hilbert manifolds) between $\mathcal{H}$ and $\mathcal{H}_{\hat{a}}(\mathcal{N})$ which preserves the energy, that is, $\mathcal{E}(\psi(h))=\|h\|_{\mathcal{H}}^{2}$ for any $h \in \mathcal{H}$. Therefore,

$$
d_{a}^{2}=\min \left\{\mathcal{E}(\xi) \mid \xi \in \mathcal{H}_{\hat{a}}(\mathcal{N}), \xi_{1}=a\right\}
$$

and

$$
\psi\left(\mathcal{K}_{a}^{\min }\right)=\left\{\xi \in \mathcal{H}_{\hat{a}}(\mathcal{N}) \mid \xi_{1}=a, \mathcal{E}(\xi)=d_{a}^{2}\right\}
$$


which will be denoted by $\mathcal{K}_{\hat{a}, a}^{\min }(\mathcal{N})$. In other words, the set of minimal energy paths does not change through $\psi$. Moreover, $d_{a}$ is equal to the Riemannian distance $d_{\mathcal{N}}(\hat{a}, a)$.

Let us rewrite the other assumptions in terms of $\mathcal{K}_{\hat{a}, a}^{\min }(\mathcal{N})$. First, (B2) is equivalent to

(R2): $\mathcal{K}_{\hat{a}, a}^{\min }(\mathcal{N})$ is a smooth and compact manifold of finite dimension $n^{\prime}$ regularly embedded in $\mathcal{H}_{\hat{a}}(\mathcal{N})$.

Set $\mathcal{K}_{\hat{a}, a}(\mathcal{N}):=\left\{\xi \in \mathcal{H}_{\hat{a}}(\mathcal{N}) \mid \xi_{1}=a\right\}$ and restrict $\mathcal{E}$ to this set. Then, (B3) is equivalent to

(R3): For any $\xi \in \mathcal{K}_{\hat{a}, a}^{\min }(\mathcal{N})$ and any $k \in T_{\xi} \mathcal{K}_{\hat{a}, a}(\mathcal{N}) \backslash T_{\xi} \mathcal{K}_{\hat{a}, a}^{\min }(\mathcal{N}), \mathcal{E}^{\prime \prime}(\xi)\langle k, k\rangle>0$. Then, as a corollary of our main theorem, we obtain the following: assume $\hat{a} \neq a$, (R2) and (R3). Then, as a special case $\left(G^{\varepsilon} \equiv 1\right)$ of Theorem 2.5, we have the following asymptotic expansion:

$$
p_{\varepsilon^{2}}(\hat{a}, a) \sim \exp \left(-\frac{d_{\mathcal{N}}(\hat{a}, a)^{2}}{2 \varepsilon^{2}}\right) \varepsilon^{-\left(n+n^{\prime}\right)}\left(c_{0}+c_{2} \varepsilon^{2}+c_{4} \varepsilon^{4}+\cdots\right) \quad \text { as } \varepsilon \searrow 0
$$

for certain constants $c_{2 j} \in \mathbb{R}(j \geqslant 0)$.

Conditions (R2) and (R3) are natural and often appear in Morse theory and Riemannian geometry (possibly in a slightly different form). Note that the dimension of the null eigenspace of $\mathcal{E}^{\prime \prime}(\xi)$ equals the dimension of the set of Jacobi fields along $\xi$ which vanish at both ends.

The simplest example which satisfies (R2) and (R3) is the sphere $\mathbb{S}^{n}$ and two antipodal points $\hat{a}$ and $a$ on it. In that case, $\mathcal{K}_{\hat{a}, a}^{\min }$ consists of great circles from $\hat{a}$ to $a$ and therefore diffeomorphic to $\mathbb{S}^{n-1}$.

REMARK 2.9. We are not aware of any preceding work that proved (2.8) above. However, a few months after we finished our present work, Ludewig [33] proved a similar theorem. His conditions on $\mathcal{K}_{\hat{a}, a}^{\min }$, which is called Morse-Bott condition in [33], are essentially equivalent to (R2) and (R3). His method is basically analytic. Compared to (2.8), his result is stronger in the following senses. First, he obtained the leading constant $c_{0}$ in two explicit ways. Second, he studied heat kernels associated with Laplace-type operators acting on sections of a vector bundle in a systematic way. On the other hand, no hypoelliptic case is studied in [33].

The next example is a compact, strictly pseudo-convex CR manifold and its unitary frame bundle in Kondo and Taniguchi [29]. For fundamental facts on CR manifolds, the reader is referred to [15] among others. 
EXAMPLE 2.10. A CR (Cauchy-Riemann) manifold $\mathcal{N}$ is a real smooth manifold equipped with a complex subbundle $T_{1,0}$ of the complexified tangent bundle $\mathbb{C} T \mathcal{N}$ with properties that $T_{1,0} \cap T_{0,1}=\{0\}$, where $T_{0,1}=\overline{T_{1,0}}$ is the complex conjugate of $T_{1,0}$ and $\left[T_{1,0}, T_{1,0}\right] \subset T_{1,0}$. Assume that $\mathcal{N}$ is compact, orientable and of real dimension $2 k+1$ and $T_{1,0}$ is of complex dimension $k$.

There exists a real nonvanishing 1-form $\theta$ on $\mathcal{N}$ annihilating $H=$ $\operatorname{Re}\left(T_{1,0} \oplus T_{0,1}\right)$. We assume that $\mathcal{N}$ is strictly pseudo-convex, that is the Levi form $L_{\theta}$ defined by

$$
L_{\theta}(Z, W)=-\sqrt{-1} d \theta(Z, W) \text { for } Z, W \in \Gamma^{\infty}\left(T_{1,0} \oplus T_{0,1}\right),
$$

where $\Gamma^{\infty}(V)$ is the space of smooth sections of vector bundle $V$, is positive definite. Associated with $\theta$, the characteristic direction $T$, the unique real vector field on $\mathcal{N}$ transverse to $H$ is given by

$$
T\rfloor d \theta=0, \quad T\rfloor \theta=1,
$$

where $T\rfloor \omega$ is the interior product.

The Webster metric $g_{\theta}$ is defined by

$g_{\theta}(X, Y)=d \theta(X, J Y), \quad g_{\theta}(X, T)=0, \quad g_{\theta}(T, T)=1 \quad$ for $X, Y \in \Gamma^{\infty}(H)$,

where $J: T \mathcal{N} \rightarrow T \mathcal{N}$ is defined so that its complex linear extension to $\mathbb{C} T \mathcal{N}$ is equal to the multiplication by $\sqrt{-1}$ on $T_{1,0}$, that by $-\sqrt{-1}$ on $T_{0,1}$, and $J(T)=0$. The Tanaka-Webster connection is a unique linear connection $\nabla$ on $\mathcal{N}$ such that

$$
\begin{array}{cl}
\nabla_{X} Y \in \Gamma^{\infty}(H), \quad \nabla J=0, & \nabla g_{\theta}=0, \quad T_{\nabla}(Z, W)=0, \\
T_{\nabla}\left(Z, W^{\prime}\right)=2 \sqrt{-1} L_{\theta}\left(Z, W^{\prime}\right) T, & T_{\nabla}(T, J(X))+J\left(T_{\nabla}(T, X)\right)=0
\end{array}
$$

for $X \in \Gamma^{\infty}(T \mathcal{N}), Y \in \Gamma^{\infty}(H), Z, W \in \Gamma^{\infty}\left(T_{1,0}\right)$, and $W^{\prime} \in \Gamma^{\infty}\left(T_{0,1}\right)$, where $\nabla_{X}$ is the covariant derivative in the direction of $X$ and $T_{\nabla}$ is the torsion tensor field of $\nabla$.

Define the unitary frame bundle over $\mathcal{N}$ by

$$
U\left(T_{1,0}\right)=\coprod_{x \in M}\left\{u: \mathbb{C}^{k} \rightarrow\left(T_{1,0}\right)_{x} ; u \text { is a unitary isometry }\right\} .
$$

For $u \in U\left(T_{1,0}\right)$ with $u: \mathbb{C}^{k} \rightarrow\left(T_{1,0}\right)_{x}$, let $\Pi(u)=x$. Every $v \in T_{x} \mathcal{N}$ admits a unique horizontal lift $\eta_{u}(v) \in T_{u} U\left(T_{1,0}\right)$, where $u \in \Pi^{-1}(x)$, so that there exist smooth curves $p:[-\tau, \tau] \rightarrow \mathcal{N}$ and $\hat{p}:[-\tau, \tau] \rightarrow U\left(T_{1,0}\right)$ such that $\Pi \circ \hat{p}=p$, $\hat{p}(0)=u, \hat{p}^{\prime}(0)=\eta_{u}(v),\left(\Pi_{*}\right)_{u} \eta_{u}(v)=v$, and the curve $[-\tau, \tau] \ni t \mapsto \hat{p}(t) \zeta \in$ $T_{1,0}$ is a parallel section along $p$ with respect to $\nabla$ for any $\zeta \in \mathbb{C}^{k}$. Extending $\eta_{u}$ naturally to a complex linear mapping of $\mathbb{C} T_{x} M$ to $T_{u} U\left(T_{1,0}\right)$, set $L(\zeta)_{u}=$ $\eta_{u}(u \zeta)$ for $\zeta \in \mathbb{C}^{k}$. Using the standard complex basis $\left\{e_{1}, \ldots, e_{k}\right\}$ of $\mathbb{C}^{k}$, define 
the canonical vector fields $L_{1}, \ldots, L_{k}$ by

$$
L_{i}=L\left(e_{i}\right), \quad i=1, \ldots, k .
$$

Let $V_{1}, \ldots, V_{2 k}$ be vector fields on $U\left(T_{1,0}\right)$ defined by

$$
V_{i}=\frac{1}{\sqrt{2}}\left(L_{i}+\overline{L_{i}}\right) \quad \text { and } \quad V_{k+i}=\frac{1}{\sqrt{2} \sqrt{-1}}\left(L_{i}-\overline{L_{i}}\right), \quad i=1, \ldots, k .
$$

Consider the stochastic differential equation on $U\left(T_{1,0}\right)$ given by

$$
d X_{t}^{\varepsilon}=\varepsilon \sum_{i=1}^{2 k} V_{i}\left(X_{t}^{\varepsilon}\right) \circ d w^{i}(t)
$$

for $0<\varepsilon \leqslant 1$. Then $Y_{t}^{\varepsilon}:=\Pi\left(X_{t}^{\varepsilon}\right)$ determines a diffusion process generated by $-\left(\varepsilon^{2} / 2\right) \triangle_{b}$. Here the operator $\triangle_{b}$ is a hypoelliptic sub-Laplacian given by

$$
\int_{\mathcal{N}}\left(\triangle_{b} f\right) g d \mathrm{vol}=\int_{\mathcal{N}} L_{\theta}^{*}\left(d_{b} f, d_{b} g\right) d \mathrm{vol}
$$

for any smooth $f, g$ on $\mathcal{N}$, where vol $=\theta \wedge(d \theta)^{k}, L_{\theta}^{*}$ is the dual metric on $H^{*}$ of $L_{\theta}$, and $d_{b} f$ is the projection of $d f$ onto $H^{*}$.

Assumption (A1)' clearly holds. Moreover (A2)' is also satisfied as was seen in [29]. Indeed, if $\left\{Z_{i}\right\}_{i=1}^{n}$ is a local orthonormal frame of $T_{1,0}$, then

$$
\left(\Pi_{*}\right)_{u}\left(L_{i}\right)=\sum_{j=1}^{k} e_{j}^{i} Z_{j} \quad \text { and }
$$

$$
\left(\Pi_{*}\right)_{u}\left[L_{i}, L_{\bar{i}}\right]=-2 \sqrt{-1} T \bmod \left\{Z_{i}, Z_{\bar{i}} \mid i=1, \ldots, k\right\},
$$

where $\left(e_{i}^{j}\right) \in U(n)$. See Kondo and Taniguchi [29] for details.

We say that an absolutely continuous path $\xi$ on $\mathcal{N}$ is horizontal in the $C R$ sense if $\xi_{t}^{\prime} \in H_{\xi_{t}}$ for almost all $t$. We denote by $\tilde{\mathcal{H}}_{\hat{a}}(\mathcal{N})$ the set of absolutely continuous path $\xi$ on $\mathcal{N}$ which is horizontal in the CR sense and start at $\hat{a}$. The energy functional $\tilde{\mathcal{E}}$ on $\tilde{\mathcal{H}}_{\hat{a}}(\mathcal{N})$ is defined in the same way as in Example 2.8, this time with the Webster metric instead of the Riemannian metric. In this case again, the development map $h \mapsto \psi(h)$ is an energy-preserving diffeomorphism (of Hilbert manifolds) between $\mathcal{H}$ and $\tilde{\mathcal{H}}_{\hat{a}}(\mathcal{N})$. Therefore,

$$
d_{a}^{2}=\min \left\{\tilde{\mathcal{E}}(\xi) \mid \xi \in \tilde{\mathcal{H}}_{\hat{a}}(\mathcal{N}), \xi_{1}=a\right\}
$$

and

$$
\psi\left(\mathcal{K}_{a}^{\min }\right)=\left\{\xi \in \tilde{\mathcal{H}}_{\hat{a}}(\mathcal{N}) \mid \xi_{1}=a, \tilde{\mathcal{E}}(\xi)=d_{a}^{2}\right\},
$$

which will be denoted by $\tilde{\mathcal{K}}_{\hat{a}, a}^{\min }(\mathcal{N})$. In other words, the set of minimal energy paths does not change through $\psi$. Moreover, $d_{a}$ is equal to the sub-Riemannian (Carnot-Carathéodory) distance $\tilde{d}_{\mathcal{N}}(\hat{a}, a)$. In a strictly pseudo-convex CR 
manifold, no nontrivial energy-minimizing path (that is geodesics) is abnormal in the sense of sub-Riemannian geometry. (See [42, pages 24-25] for example.) Hence, (B1) is always satisfied.

Let us rewrite the other assumptions in terms of $\tilde{\mathcal{K}}_{\hat{a}, a}^{\min }(\mathcal{N})$. First, (B2) is equivalent to

(CR2): $\tilde{\mathcal{K}}_{\hat{a}, a}^{\min }(\mathcal{N})$ is a smooth and compact manifold of finite dimension $n^{\prime}$ regularly embedded in $\tilde{\mathcal{H}}_{\hat{a}}(\mathcal{N})$.

Set $\tilde{\mathcal{K}}_{\hat{a}, a}(\mathcal{N}):=\left\{\xi \in \tilde{\mathcal{H}}_{\hat{a}}(\mathcal{N}) \mid \xi_{1}=a\right\}$ and restrict $\tilde{\mathcal{E}}$ to this set. Then, (B3) is equivalent to

(CR3): For any $\xi \in \tilde{\mathcal{K}}_{\hat{a}, a}^{\min }(\mathcal{N})$ and any $l \in T_{\xi} \tilde{\mathcal{K}}_{\hat{a}, a}(\mathcal{N}) \backslash T_{\xi} \tilde{\mathcal{K}}_{\hat{a}, a}^{\min }(\mathcal{N}), \tilde{\mathcal{E}}^{\prime \prime}(\xi)\langle l, l\rangle$ $>0$.

Then, as a corollary of our main theorem, we obtain the following: assume $\hat{a} \neq a$, (CR2) and (CR3). Then, as a special case $\left(G^{\varepsilon} \equiv 1\right)$ of Theorem 2.5, we have the following asymptotic expansion:

$$
\tilde{p}_{\varepsilon^{2}}(\hat{a}, a) \sim \exp \left(-\frac{\tilde{d}_{\mathcal{N}}(\hat{a}, a)^{2}}{2 \varepsilon^{2}}\right) \varepsilon^{-\left(2 k+1+n^{\prime}\right)}\left(c_{0}+c_{2} \varepsilon^{2}+c_{4} \varepsilon^{4}+\cdots\right) \quad \text { as } \varepsilon \searrow 0
$$

for certain constants $c_{2 j} \in \mathbb{R}(j \geqslant 0)$. Here, $\tilde{p}$ stands for the heat kernel associated with the sub-Laplacian $\triangle_{b} / 2$.

The next example is a continuation of Example 2.10 above. We provide a very concrete example of $\mathcal{N}$ and $\hat{a}, a \in \mathcal{N}$ which satisfy (CR2) and (CR3).

EXAMPLE 2.11. In this example, we show that antipodal points on the standard CR sphere $\mathbb{S}^{2 k+1}(k \geqslant 1)$ satisfy (CR2) and (CR3). For basics of the standard CR sphere, we refer to $[5,13,39]$. To keep notations simple, we avoid the complex coordinates and describe the standard CR sphere as a real manifold.

Let $\mathbb{S}^{2 k+1}=\left\{\left(x_{0}, y_{0}, \ldots, x_{k}, y_{k}\right) \in \mathbb{R}^{2 k+2} \mid \sum_{i=0}^{k}\left(x_{i}^{2}+y_{i}^{2}\right)=1\right\}$. It has a natural strictly pseudo-convex CR structure inherited from $\mathbb{C}^{k+1} \cong \mathbb{R}^{2 k+2}$, which is given as follows:

$$
\begin{aligned}
& \left(T_{1,0}\right)_{\left(x_{0}, y_{0}, \ldots, x_{k}, y_{k}\right)} \\
& =\left\{\sum_{i=0}^{k}\left[\left(p_{i} \frac{\partial}{\partial x_{i}}+q_{i} \frac{\partial}{\partial y_{i}}\right)+\sqrt{-1}\left(q_{i} \frac{\partial}{\partial x_{i}}-p_{i} \frac{\partial}{\partial y_{i}}\right)\right] \mid\right. \\
& \left.\left(p_{0}, q_{0}, \ldots, p_{k}, q_{k}\right) \in \mathbb{R}^{2 k+2}, \sum_{i=0}^{k}\left(p_{i} x_{i}+q_{i} y_{i}\right)=0, \sum_{i=0}^{k}\left(q_{i} x_{i}-p_{i} y_{i}\right)=0\right\} \text {. }
\end{aligned}
$$


The horizontal subbundle $H$ coincides with the kernel of the following contact one-form: $\omega=\sum_{i=0}^{k}\left(-y_{i} d x_{i}+x_{i} d y_{i}\right)$. The Webster metric on $\mathbb{S}^{2 k+1}$ is just restriction of the canonical one on $\mathbb{R}^{2 k+2}$.

We choose $( \pm 1,0, \ldots, 0,0)$ as the starting point and the end point, respectively, and denote them by $\mathbf{e}$ and $-\mathbf{e}$. By the way the metric is defined, we have $\mathcal{E}(\xi)=$ $\tilde{\mathcal{E}}(\xi)$ for a horizontal path $\xi$ on $\mathbb{S}^{2 k+1}$. In particular, we have

$$
\tilde{\mathcal{K}}_{\mathbf{e},-\mathbf{e}}^{\min }\left(\mathbb{S}^{2 k+1}\right)=\left\{\xi \in \mathcal{K}_{\mathbf{e},-\mathbf{e}}^{\min }\left(\mathbb{S}^{2 k+1}\right) \mid \xi \text { is horizontal in the CR sense }\right\} .
$$

As we see, the right hand side above is not empty. The sub-Riemannian distance between the two points is $\pi$.

Recall that $\mathcal{K}_{\mathbf{e},-\mathbf{e}}^{\min }\left(\mathbb{S}^{2 k+1}\right)$ is the set of all the great circles from $\mathbf{e}_{+}$to $\mathbf{e}_{-}$(in time 1). We write them down explicitly. Set

$$
\begin{gathered}
\mathbb{S}^{2 k}=\left\{\left(p_{0}, q_{0}, \ldots, p_{k}, q_{k}\right) \in \mathbb{S}^{2 k+1} \mid p_{0}=0\right\}, \\
\mathbb{S}^{2 k-1}=\left\{\left(p_{0}, q_{0}, \ldots, p_{k}, q_{k}\right) \in \mathbb{S}^{2 k+1} \mid p_{0}=q_{0}=0\right\} .
\end{gathered}
$$

For $\mathbf{v}=\left(0, q_{0}, \ldots, p_{k}, q_{k}\right) \in \mathbb{S}^{2 k}$, we write

$$
\xi_{\mathbf{v}}(t)=\cos (\pi t) \mathbf{e}+\sin (\pi t) \mathbf{v} .
$$

Then, $\xi_{\mathrm{v}}$ is a great circle and we have

$$
\mathcal{K}_{\mathbf{e},-\mathbf{e}}^{\min }\left(\mathbb{S}^{2 k+1}\right)=\left\{\xi_{\mathbf{v}} \mid \mathbf{v} \in \mathbb{S}^{2 k}\right\}
$$

Moreover, by straightforward computation, we have $\left\langle\xi_{\mathrm{v}}^{\prime}, \omega_{\xi_{\mathrm{v}}}\right\rangle \equiv \pi q_{0}$. Hence, $\xi_{\mathrm{v}}$ is horizontal in the CR sense if and only if $q_{0}=0$ and we have

$$
\tilde{\mathcal{K}}_{\mathbf{e},-\mathbf{e}}^{\min }\left(\mathbb{S}^{2 k+1}\right)=\left\{\xi_{\mathbf{v}} \mid \mathbf{v} \in \mathbb{S}^{2 k-1}\right\} .
$$

Thus, we have shown (CR2) with $n^{\prime}=2 k-1$.

Now we turn to (CR3). A key fact is

$$
T_{\xi} \tilde{\mathcal{K}}_{\mathbf{e},-\mathbf{e}}^{\min }\left(\mathbb{S}^{2 k+1}\right)=T_{\xi} \mathcal{K}_{\mathbf{e},-\mathbf{e}}^{\min }\left(\mathbb{S}^{2 k+1}\right) \cap T_{\xi} \tilde{\mathcal{K}}_{\mathbf{e},-\mathbf{e}}\left(\mathbb{S}^{2 k+1}\right) \quad\left(\xi \in \tilde{\mathcal{K}}_{\mathbf{e},-\mathbf{e}}^{\min }\left(\mathbb{S}^{2 k+1}\right)\right) .
$$

Once (2.10) is established, the problem reduces to the corresponding one for $\mathbb{S}^{2 k+1}$ as a Riemannian manifold. The reason is as follows. If $l \in T_{\xi} \tilde{\mathcal{K}}_{\mathbf{e},-\mathbf{e}}\left(\mathbb{S}^{2 k+1}\right) \backslash$ $T_{\xi} \tilde{\mathcal{K}}_{\mathbf{e},-\mathbf{e}}^{\min }\left(\mathbb{S}^{2 k+1}\right)$, then $l \in T_{\xi} \mathcal{K}_{\mathbf{e},-\mathbf{e}}\left(\mathbb{S}^{2 k+1}\right) \backslash T_{\xi} \mathcal{K}_{\mathbf{e},-\mathbf{e}}^{\min }\left(\mathbb{S}^{2 k+1}\right)$ by $(2.10)$. We have already seen in the paragraph just above Remark 2.9 that $\tilde{\mathcal{E}}^{\prime \prime}(\xi)\langle l, l\rangle=\mathcal{E}^{\prime \prime}(\xi)\langle l, l\rangle$ $>0$ holds for such $l$. This proves (CR3).

It is obvious that the left hand side of (2.10) is included in the right hand side. To see the converse inclusion, we use $F: \mathcal{K}_{\mathbf{e},-\mathbf{e}}\left(\mathbb{S}^{2 k+1}\right) \rightarrow \mathbb{R}$ defined by $F(\xi)=$ $\int_{0}^{1}\left\langle\xi^{\prime}(t), \omega_{\xi(t)}\right\rangle d t$. Let $(-1,1) \ni \tau \mapsto c(\tau) \in \mathcal{K}_{\mathbf{e},-\mathbf{e}}\left(\mathbb{S}^{2 k+1}\right)$ be a smooth curve such 
that $c(0)=\xi \in \tilde{\mathcal{K}}_{\mathbf{e},-\mathbf{e}}^{\min }\left(\mathbb{S}^{2 k+1}\right)$. Then, $\tau \mapsto c(\tau)^{\prime}$ is a smooth curve in $L^{2}([0,1]$, $\mathbb{R}^{2 k+2}$ ) since the differentiation in $t$ (denoted by 'prime') is a unitary isomorphism from the $\left(\mathbb{R}^{2 k+2}\right.$-valued) Cameron-Martin space to $L^{2}\left([0,1], \mathbb{R}^{2 k+2}\right)$. It is easy to see that $\left.(d / d \tau)\right|_{\tau=0} F(c(\tau))$ exists. Moreover, it depends only on $\xi=c(0)$ and $l:=\left.(d / d \tau)\right|_{\tau=0} c(\tau)$. (The latter can be regarded as a Cameron-Martin path in $\mathbb{R}^{2 k+2}$.) It is obvious that $\left.(d / d \tau)\right|_{\tau=0} F(c(\tau))=0$ if $l \in T_{\xi} \tilde{\mathcal{K}}_{\mathbf{e},-\mathbf{e}}\left(\mathbb{S}^{2 k+1}\right)$.

However, we can show that $\left.(d / d \tau)\right|_{\tau=0} F(c(\tau)) \neq 0$ if $l \in T_{\xi} \mathcal{K}_{\mathbf{e},-\mathbf{e}}^{\min }\left(\mathbb{S}^{2 k+1}\right) \backslash$ $T_{\xi} \tilde{\mathcal{K}}_{\mathbf{e},-\mathbf{e}}^{\min }\left(\mathbb{S}^{2 k+1}\right)$ as follows. Let $\mathbf{v}=\left(0,0, p_{1}, q_{1}, \ldots, p_{k}, q_{k}\right) \in \mathbb{S}^{2 k-1}$ be such that $\xi=\xi_{\mathbf{v}}$ and let $\tau \mapsto \mathbf{v}(\tau)=\left(0, q_{0}(\tau), \ldots, p_{k}(\tau), q_{k}(\tau)\right)$ be a smooth curve in $\mathbb{S}^{2 k}$ such that $\mathbf{v}(0)=\mathbf{v}$. It is easy to see that $\left.(d / d \tau)\right|_{\tau=0} \mathbf{v}(\tau)$ is tangent to $\mathbb{S}^{2 k-1}$ at $\mathbf{v}$ if and only if $\left.(d / d \tau)\right|_{\tau=0} q_{0}(\tau)=0$. As we have seen, $\left.(d / d \tau)\right|_{\tau=0} F\left(\xi_{\mathbf{v}(\tau)}\right)=$ $\left.\pi(d / d \tau)\right|_{\tau=0} q_{0}(\tau)$. This proves our claim in this paragraph and hence (2.10), too.

Finally, we show that the sub-Laplacian $\triangle_{b}$ on $\mathbb{S}^{2 k+1}$ cannot be written in a 'sum of squares' form of exactly $2 k$ vector fields if $2 k+1 \neq 3$ or 7 , that is, we cannot find vector fields $A_{i}(0 \leqslant i \leqslant 2 k)$ on $\mathbb{S}^{2 k+1}$ such that $\triangle_{b}=\sum_{i=1}^{2 k} A_{i}^{2}+$ $A_{0}$. (We should recall that $2 k$ is the real dimension of $T_{1,0}$.) Consequently, we need the stochastic parallel transport as in [29] to construct the diffusion process generated by $-\triangle_{b} / 2$ (as long as we use $2 k$-dimensional Brownian motion). The reason why $\triangle_{b}$ cannot be written in such a form is as follows. If it could, by a formula $\triangle_{L B}=\triangle_{b}+T^{2}$ in [5, page 137], the Laplace-Beltrami operator $\triangle_{L B}$ of $\mathbb{S}^{2 k+1}$ as a Riemannian manifold would have a 'sum of squares' form $\triangle_{L B}=$ $\sum_{i=1}^{2 k} A_{i}^{2}+T^{2}+A_{0}$. However, this is impossible if $2 k+1 \neq 3,7$. $\left(\mathbb{S}^{j}\right.$ is not parallelizable for $j \neq 1,3,7$ and therefore $\left\{A_{1}, \ldots, A_{2 k}, T\right\}$ cannot be a global frame of the tangent bundle of $\mathbb{S}^{2 k+1}$.)

In [5, Proposition 3.7], the leading term of short time asymptotic of $\tilde{p}_{t}(\mathbf{e}, \mathbf{f})$ is calculated with an explicit value of the leading constant $c_{0}$, where $\mathbf{f}=$ $\left(e^{i \theta}, 0, \ldots, 0\right) \in \mathbb{C}^{n+1} \cong \mathbb{R}^{2 n+2}(0<\theta<\pi)$. However, the case $\theta=\pi$ (that is $\mathbf{f}=-\mathbf{e})$ seems to be excluded. Since all the energy minimizers on the standard CR sphere is obtained in $[13,39]$, it may be an interesting future task to strengthen [5, Proposition 3.7] by combining our result (2.9) with [13, 39].

Grong and Thalmaier [18] recently showed that diffusion processes on subRiemannian manifolds associated with sub-Laplacians also admit a similar construction. For basic information on sub-Riemannian geometry, we refer to $[11,40,42]$ among others.

EXAMPLE 2.12 (Diffusions associated with sub-Laplacians on sub-Riemannian manifolds. See [18, Section 2] for details). Let $(\mathcal{N}, \mathcal{D}, \mathbf{g})$ be a compact subRiemannian manifold. Here, $\mathcal{D}$ is a subbundle of the tangent bundle $T \mathcal{N}$ of a 
smooth compact manifold $\mathcal{N}$ with $\operatorname{dim} \mathcal{N}=n$ and $\mathbf{g}$ is a metric tensor on $\mathcal{D}$. By definition, $\mathcal{D}$ is bracket generating.

Let $\overline{\mathbf{g}}$ be a Riemannian metric tensor of $\mathcal{N}$ that tames $\mathbf{g}$ (that is $\left.\overline{\mathbf{g}}\right|_{\mathcal{D}}=\mathbf{g}$ ). Let $\Gamma$ be the orthogonal complement of $\mathcal{D}$ in $T \mathcal{N}$. We write the orthogonal projection by $\operatorname{pr}_{\mathcal{D}}$ and $\operatorname{pr}_{\Gamma}$, respectively. Denote by $\bar{\nabla}$ the Levi-Civita connection on $\mathcal{N}$ with respect to $\overline{\mathbf{g}}$. Define a sub-Laplacian by using a local orthonormal frame $\left\{A_{1}, \ldots\right.$, $\left.A_{r}\right\}$ of $\mathcal{D}$ by

$$
\triangle_{\text {sub }}=\sum_{i=1}^{r} A_{i}^{2}+\sum_{i, j=1}^{r} \mathbf{g}\left\langle\mathrm{pr}_{\mathcal{D}} \bar{\nabla}_{A_{i}} A_{j}, A_{i}\right\rangle A_{i}
$$

where $r(1<r<n)$ is the rank of $\mathcal{D}$. This is a globally well-defined differential operator and known to be hypoelliptic.

The diffusion process on $\mathcal{N}$ associated with $\triangle_{\text {sub }} / 2+A_{0}$ admits EellsElworthy's construction for any vector field $A_{0}$ on $\mathcal{N}\left(A_{0}\right.$ need not be a section of $\mathcal{D}$.) In this case, the principle bundle is

$$
O(\mathcal{D}) \odot O(\Gamma)=\bigcup_{y \in \mathcal{N}}\left\{(u, v) \mid u: \mathbb{R}^{r} \rightarrow \mathcal{D}_{y} \text { and } v: \mathbb{R}^{n-r} \rightarrow \Gamma_{y} \text { are isometries }\right\}
$$

with its structure group $O(r) \times O(n-r)$. The projection is denoted by $\Pi$. Since $\bar{\nabla}$ is a metric connection, it defines a Cartan-Ehresmann connection on $O(\mathcal{D}) \odot$ $O(\Gamma)$. Define canonical horizontal vector fields $\bar{A}_{i}(1 \leqslant i \leqslant r)$ on $O(\mathcal{D}) \odot O(\Gamma)$ so that $\left(\bar{A}_{i}\right)_{(u, v)}$ is the horizontal lift of $u e_{i} \in \mathcal{D}_{\Pi(u, v)}$, where $\left\{e_{i}\right\}_{i=1}^{r}$ is the canonical basis of $\mathbb{R}^{r}$. Also define $\overline{A_{0}}$ to be the horizontal lift of $A_{0}$. Then, the solution to SDE on $O(\mathcal{D}) \odot O(\Gamma)$ with the coefficient vector fields $\bar{A}_{i}(0 \leqslant i \leqslant r)$ is the diffusion process associated with $\triangle_{\text {sub }} / 2+A_{0}$. (In [18], only the case $A_{0} \equiv 0$ is treated. However, a modification of this first order term is easy.)

In Example 2.12 above, an example of a submersion $\Pi: \mathcal{M} \rightarrow \mathcal{N}$ and vector fields which satisfy (A1) and (A2) is given. However, as readers may have noticed, there is no concrete example of a sub-Riemannian manifold and two points on it. (In this sense this should be called a potential example.) It is an interesting and important future task to find such examples which satisfies our assumptions (B1)-(B3).

REMARK 2.13. Our present paper is based on Takanobu and Watanabe [47] and the assumptions are quite similar. In particular, the assumptions on the set of energy minimizers are the exactly same. Our main result is stronger than the one in [47] because we work under the partial Hörmander condition and work also on manifold. 
On the other hand, Barilari et al. [3] imposes assumptions on the set of energy minimizers which look quite different from our (B1)-(B3) and obtained the leading term of the asymptotics.

The relation between the two types of assumptions are unclear, yet. Neither is it clear at the moment whether a full asymptotic expansion can be proved under the assumptions in [3].

\section{Preliminaries}

In this section we summarize results we use in the proof of our main theorems. All the results in this section are either known or easily derived from known results.

3.1. Preliminaries from Malliavin calculus. We first recall Watanabe's theory of generalized Wiener functionals (that is Watanabe distributions) in Malliavin calculus. Most of the contents and the notations in this subsection are contained in Ikeda and Watanabe [22, Sections V.8-V.10] with trivial modifications. We also refer to Shigekawa [44], Nualart [41], Hu [20] and Matsumoto and Taniguchi [37]. For basic results of quasisure analysis, see Malliavin [36, Ch. II].

Let $(\mathcal{W}, \mathcal{H}, \mu)$ be the classical Wiener space as before. (The results in this subsection also hold on any abstract Wiener space, however.) We denote by $D$ the gradient operator ( $\mathcal{H}$-derivative) and by $L=-D^{*} D$ the Ornstein-Uhlenbeck operator. The following are of particular importance in this paper:

(a) Basics of Sobolev spaces: we denote by $\mathbb{D}_{p, r}(\mathcal{X})$ the Sobolev space of $\mathcal{X}$-valued (generalized) Wiener functionals, where $p \in(1, \infty), r \in \mathbb{R}$, and $\mathcal{X}$ is a real separable Hilbert space. As usual, we use the spaces $\mathbb{D}_{\infty}(\mathcal{X})=\bigcap_{k=1}^{\infty} \bigcap_{1<p<\infty} \mathbb{D}_{p, k}(\mathcal{X}), \tilde{\mathbb{D}}_{\infty}(\mathcal{X})=\bigcap_{k=1}^{\infty} \bigcup_{1<p<\infty} \mathbb{D}_{p, k}(\mathcal{X})$ of test functionals and the spaces $\mathbb{D}_{-\infty}(\mathcal{X})=\bigcup_{k=1}^{\infty} \bigcup_{1<p<\infty} \mathbb{D}_{p,-k}(\mathcal{X}), \tilde{\mathbb{D}}_{-\infty}(\mathcal{X})=$ $\bigcup_{k=1}^{\infty} \bigcap_{1<p<\infty} \mathbb{D}_{p,-k}(\mathcal{X})$ of Watanabe distributions as in [22]. When $\mathcal{X}=\mathbb{R}$, we simply write $\mathbb{D}_{p, r}$, and so forth. The $\mathbb{D}_{p, r}(\mathcal{X})$-norm is denoted by $\|\cdot\|_{p, r}$. The precise definition of an asymptotic expansion up to any order can be found in [22, Section V-9].

(b) Meyer's equivalence of Sobolev norms: see [22, Theorem 8.4]. A stronger version can be found in [44, Theorem 4.6], in [41, Theorem 1.5.1] or in Bogachev [9, Theorem 5.7.1].

(c) Watanabe's pullback: pullback $T \circ F=T(F) \in \tilde{\mathbb{D}}_{-\infty}$ of a tempered Schwartz distribution $T \in \mathcal{S}^{\prime}\left(\mathbb{R}^{n}\right)$ on $\mathbb{R}^{n}$ by a non-degenerate Wiener functional 
$F \in \mathbb{D}_{\infty}\left(\mathbb{R}^{n}\right)$. (See [22, Section 5.9].) The key to prove this pullback is an integration by parts formula in the sense of Mallavin calculus. (Its generalization is given in Item (d) below.)

(d) A generalized version of the integration by parts formula in the sense of Malliavin calculus for Watanabe distribution, which is given as follows (see [22, page 377]):

For a non-degenerate Wiener functional $F=\left(F^{1}, \ldots, F^{n}\right) \in \mathbb{D}_{\infty}\left(\mathbb{R}^{n}\right)$, we denote by $\sigma[F](w)=\sigma_{F}(w)$ the Malliavin covariance matrix of $F$ whose $(i, j)$-component is given by $\sigma_{F}^{i j}(w)=\left\langle D F^{i}(w), D F^{j}(w)\right\rangle_{\mathcal{H}}$. We denote by $\gamma_{F}^{i j}(w)$ the $(i, j)$-component of the inverse matrix $\sigma_{F i j}^{-1}$. Note that $\sigma_{F}^{i j} \in \mathbb{D}_{\infty}$ and $D \gamma_{F}^{i j}=-\sum_{k, l} \gamma_{F}^{i k}\left(D \sigma_{F}^{k l}\right) \gamma_{F}^{l j}$. Hence, derivatives of $\gamma_{F}^{i j}$ can be written in terms of $\gamma_{F}^{i j}$,s and the derivatives of $\sigma_{F}^{i j}$ 's. Suppose $G \in \mathbb{D}_{\infty}$ and $T \in \mathcal{S}^{\prime}\left(\mathbb{R}^{n}\right)$. Then, the following integration by parts holds;

$$
\mathbb{E}\left[\left(\partial_{i} T \circ F\right) G\right]=\mathbb{E}\left[(T \circ F) \Phi_{i}(\cdot ; G)\right],
$$

where $\Phi_{i}(w ; G) \in \mathbb{D}_{\infty}$ is given by

$$
\begin{aligned}
\Phi_{i}(w ; G)= & \sum_{j=1}^{d} D^{*}\left(\gamma_{F}^{i j}(w) G(w) D F^{j}(w)\right) \\
= & -\sum_{j=1}^{d}\left\{-\sum_{k, l=1}^{d} G(w) \gamma_{F}^{i k}(w) \gamma_{F}^{j l}(w)\left\langle D \sigma_{F}^{k l}(w), D F^{j}(w)\right\rangle_{\mathcal{H}}\right. \\
& \left.+\gamma_{F}^{i j}(w)\left\langle D G(w), D F^{j}(w)\right\rangle_{\mathcal{H}}+\gamma_{F}^{i j}(w) G(w) L F^{j}(w)\right\} .
\end{aligned}
$$

Note that the expectations in (3.1) are in fact the generalized ones, that is the pairing of $\tilde{\mathbb{D}}_{-\infty}$ and $\tilde{\mathbb{D}}_{\infty}$.

Watanabe's asymptotic expansion theorem is a key theorem in his distributional Malliavin calculus. Its standard version can be found in [22, Theorem 9.4, pages 387-388] or Watanabe [52]. In the present paper, however, we need a modified version in [47, pages 216-217]. Though it plays a key role, no proof is given in [47] unfortunately. Therefore, we prove it below.

Let $\rho>0, \xi \in \mathbb{D}_{\infty}$ and $F \in \mathbb{D}_{\infty}\left(\mathbb{R}^{n}\right)$ and suppose that

$$
\inf _{v \in \mathbb{R}^{n}|| v \mid=1} v^{*} \sigma_{F}(w) v \geqslant \rho \quad \text { on }\{w \in \mathcal{W}|| \xi(w) \mid \leqslant 2\} .
$$

Let $\chi: \mathbb{R} \rightarrow \mathbb{R}$ be a smooth function whose support is contained in $[-1,1]$. Then, the following proposition holds [47, Proposition 6.1]. 
Proposition 3.1. Assume (3.3). For every $T \in \mathcal{S}^{\prime}\left(\mathbb{R}^{n}\right), \chi(\xi)(T \circ F)=$ $\chi(\xi) T(F) \in \tilde{\mathbb{D}}_{-\infty}$ can be defined in a unique way so that the following properties hold:

(i) if $T_{k} \rightarrow T \in \mathcal{S}^{\prime}\left(\mathbb{R}^{n}\right)$ as $k \rightarrow \infty$, then $\chi(\xi) T_{k}(F) \rightarrow \chi(\xi) T(F) \in \tilde{\mathbb{D}}_{-\infty}$;

(ii) if $T$ is given by $g \in \mathcal{S}\left(\mathbb{R}^{n}\right)$, then $\chi(\xi) T(F)=\chi(\xi) g(F) \in \mathbb{D}_{\infty}$.

Proof. Let $\eta: \mathbb{R} \rightarrow \mathbb{R}$ be a smooth function whose support is contained in $(-2,2)$. By the assumption (3.3), $\sigma_{F}$ is invertible on $\{\eta(\xi) \neq 0\}$ and $\eta(\xi) \sigma_{F}^{-1}$ is of class $\mathbb{D}_{\infty}$. In fact, it is the limit of $\eta(\xi)\left(\sigma_{F}+(1 / m) \operatorname{Id}_{n}\right)^{-1}$ in $\mathbb{D}_{\infty}$ as $m \rightarrow \infty$, where $\operatorname{Id}_{n}$ stands for the $n \times n$-identity matrix. Hence $\Phi_{i}(\cdot ; \eta(\xi) G)$ in (3.2) is well defined and of class $\mathbb{D}_{\infty}$. Moreover, observe that

$$
\Phi_{i}(\cdot ; \eta(\xi) G)=D^{*}\left(\eta(\xi) G \sum_{k=1}^{n} \gamma_{F}^{i k} D F^{k}\right)
$$

for any $G \in \mathbb{D}_{\infty}$. Let $f \in \mathcal{S}\left(\mathbb{R}^{n}\right)$. Since

$$
\eta(\xi)\left(\partial_{i} f \circ F\right)=\eta(\xi) \sum_{k=1}^{n}\left\langle D(f \circ F), D F^{k}\right\rangle_{\mathcal{H}} \gamma_{F}^{i k},
$$

we have the same integration by parts formula as (3.1);

$$
\mathbb{E}\left[\eta(\xi)\left(\partial_{i} f \circ F\right) G\right]=\mathbb{E}\left[(f \circ F) \Phi_{i}(\cdot ; \eta(\xi) G)\right] .
$$

Furthermore, for $p>1$ and $r>0$, let $C_{p, r}$ be a constant such that

$$
\left\|D^{*}(G K)\right\|_{p, r} \leqslant C_{p, r}\|G\|_{2 p, r+1}\|K\|_{2 p, r+1}
$$

for any $G \in \mathbb{D}_{2 p, r+1}$ and $K \in \mathbb{D}_{2 p, r+1}(\mathcal{H})$. Then

$$
\left\|\Phi_{i}(\cdot ; \eta(\xi) G)\right\|_{p, r} \leqslant C_{p, r}\left\|\eta(\xi) \sum_{k=1}^{n} \gamma_{F}^{i k} D F^{k}\right\|_{2 p, r+1}\|G\|_{2 p, r+1} .
$$

Take a sequence $\left\{\chi_{k}\right\}_{k=1}^{\infty}$ of smooth functions on $\mathbb{R}$ such that $\chi_{1}=\chi$, supp $\chi_{k} \subset$ $(-2,2)$, and $\chi_{k+1}=1$ on $\operatorname{supp} \chi_{k}, k=1,2, \ldots$ For a multi-index $\alpha=\left(\alpha_{1}\right.$, $\ldots, \alpha_{n}$ ), where $\alpha_{i}$ 's are non-negative integers, let $i_{\alpha}=\max \left\{i ; \alpha_{i} \neq 0\right\}$ and $\alpha^{\prime}=$ $\left(\alpha_{1}-\delta_{1 i_{\alpha}}, \ldots, \alpha_{n}-\delta_{n i_{\alpha}}\right), \delta_{i j}$ being Kronecker's delta. Define $\Phi_{(\alpha)}$ by

$$
\Phi_{(\alpha)}(\cdot ; G)=\Phi_{i_{\alpha}}\left(\cdot ; \chi_{1}(\xi) G\right)
$$

if $|\alpha|=\sum_{k=1}^{n} \alpha_{k}=1$, and

$$
\Phi_{(\alpha)}(\cdot ; G)=\Phi_{i_{\alpha}}\left(\cdot ; \chi_{|\alpha|}(\xi) \Phi_{\left(\alpha^{\prime}\right)}(\cdot ; G)\right)
$$

if $|\alpha| \geqslant 2$. 
It then holds that

$$
\mathbb{E}\left[\chi(\xi)\left(\partial^{\alpha} f \circ F\right) G\right]=\mathbb{E}\left[(f \circ F) \Phi_{(\alpha)}(\cdot ; G)\right]
$$

for any $f \in \mathcal{S}\left(\mathbb{R}^{n}\right)$ and $G \in \mathbb{D}_{\infty}$, where $\partial^{\alpha}=\left(\partial_{1}\right)^{\alpha_{1}} \ldots\left(\partial_{n}\right)^{\alpha_{n}}$. In fact, for $|\alpha|=1$, (3.7) is nothing but (3.5). Suppose that (3.7) holds for $\alpha$ with $|\alpha|=k$. Then for $\alpha$ with $|\alpha|=k+1$, since

$$
\chi_{|\alpha|}(\xi) \Phi_{\left(\alpha^{\prime}\right)}(\cdot ; G)=\Phi_{\left(\alpha^{\prime}\right)}(\cdot ; G),
$$

by the assumption of induction and (3.5), we obtain

$$
\begin{aligned}
\mathbb{E}\left[\chi(\xi)\left(\partial^{\alpha} f \circ F\right) G\right] & =\mathbb{E}\left[\chi(\xi)\left(\partial^{\alpha^{\prime}}\left(\partial_{i_{\alpha}} f\right) \circ F\right) G\right] \\
& =\mathbb{E}\left[\left(\partial_{i_{\alpha}} f \circ F\right) \Phi_{\left(\alpha^{\prime}\right)}(\cdot ; G)\right] \\
& =\mathbb{E}\left[\chi_{|\alpha|}(\xi)\left(\partial_{i_{\alpha}} f \circ F\right) \Phi_{\left(\alpha^{\prime}\right)}(\cdot ; G)\right] \\
& =\mathbb{E}\left[(f \circ F) \Phi_{(\alpha)}(\cdot ; G)\right] .
\end{aligned}
$$

On account of (3.6) and (3.7), repeating the standard argument to construct Watanabe's pullback in [22, Section V-9], we arrive at the unique existence of the continuous mapping $u: \mathcal{S}^{\prime}\left(\mathbb{R}^{n}\right) \rightarrow \tilde{\mathbb{D}}_{-\infty}$ such that $u(f)=\chi(\xi) f(F)$ for $f \in$ $\mathcal{S}\left(\mathbb{R}^{n}\right)$. Rewriting $u(T)$ as $\chi(\xi) T(F)$, we obtain the desired continuous linear mapping.

Next, we state the asymptotic expansion theorem, which is [47, Proposition 6.2]. Let $\left\{F_{\varepsilon}\right\}_{0<\varepsilon \leqslant 1} \subset \mathbb{D}_{\infty}\left(\mathbb{R}^{n}\right)$ and $\left\{\xi_{\varepsilon}\right\}_{0<\varepsilon \leqslant 1} \subset \mathbb{D}_{\infty}$ be families of Wiener functionals such that the following asymptotics hold:

$$
\begin{gathered}
F_{\varepsilon} \sim f_{0}+\varepsilon f_{1}+\varepsilon^{2} f_{2}+\cdots \quad \text { in } \mathbb{D}_{\infty}\left(\mathbb{R}^{n}\right) \text { as } \varepsilon \searrow 0 \\
\xi_{\varepsilon} \sim a_{0}+\varepsilon a_{1}+\varepsilon^{2} a_{2}+\cdots \quad \text { in } \mathbb{D}_{\infty} \text { as } \varepsilon \searrow 0 .
\end{gathered}
$$

Proposition 3.2. Assume (3.8), (3.9) and $\left|a_{0}\right| \leqslant 1 / 8$. Moreover, assume that there exists $\rho>0$ independent of $\varepsilon$ such that (3.3) with $F=F_{\varepsilon}$ and $\xi=\xi_{\varepsilon}$ holds for any $\varepsilon \in(0,1]$. Let $\chi: \mathbb{R} \rightarrow \mathbb{R}$ be a smooth function whose support is contained in $[-1,1]$ such that $\chi(x)=1$ if $|x| \leqslant 1 / 2$. Then, we have the following asymptotic expansion:

$$
\chi\left(\xi_{\varepsilon}\right) T\left(F_{\varepsilon}\right) \sim \Phi_{0}+\varepsilon \Phi_{1}+\varepsilon^{2} \Phi_{2}+\cdots \quad \text { in } \tilde{\mathbb{D}}_{-\infty} \text { as } \varepsilon \searrow 0 .
$$

In the above proposition, $\Phi_{k} \in \tilde{\mathbb{D}}_{-\infty}$ can be written as the $k$ th coefficient of the formal Taylor expansion of $T\left(f_{0}+\left[\varepsilon f_{1}+\varepsilon^{2} f_{2}+\cdots\right]\right)$. In particular, $\Phi_{0}=T\left(f_{0}\right)$. 
Proof. Let $T \in \mathcal{S}^{\prime}\left(\mathbb{R}^{n}\right)$. Take an $m \in \mathbb{N}$ so that $\phi=\left(1+|x|^{2}-\frac{1}{2} \Delta\right)^{-m} T$ is a bounded function on $\mathbb{R}^{n}$ which is $k$-times continuous differentiable with bounded derivatives up to order $k$. By virtue of (3.7), there exists a continuous linear mapping $\ell_{\varepsilon}: \mathbb{D}_{\infty} \rightarrow \mathbb{D}_{\infty}$ such that

$$
\mathbb{E}\left[\chi\left(\xi_{\varepsilon}\right) T\left(F_{\varepsilon}\right) G\right]=\mathbb{E}\left[\phi\left(F_{\varepsilon}\right) \ell_{\varepsilon}(G)\right] \text { for every } G \in \mathbb{D}_{\infty} .
$$

By (3.4),

$$
\ell_{\varepsilon}(G)=\sum_{i=0}^{2 m}\left\langle P_{i}(\varepsilon), D^{i} G\right\rangle_{\mathcal{H}^{\otimes i}}
$$

where $P_{i}(\varepsilon) \in \mathbb{D}_{\infty}, i=0, \ldots, 2 m$, are polynomials in $F_{\varepsilon}, \chi_{k}\left(\xi_{\varepsilon}\right) \sigma_{F_{\varepsilon}}^{-1}, k=1, \ldots$, $2 m$, and their derivatives. Since

$$
\lim _{\varepsilon \rightarrow 0} \frac{1}{\varepsilon^{k}}\left\|\chi\left(\xi_{\varepsilon}\right)-1\right\|_{p, r}=0
$$

for any $p>1, r>0$ and $k \in \mathbb{N}$, applying the argument used in the proof of [22, Theorem V.9.4] to $\chi\left(\xi_{\varepsilon}\right) \phi\left(F_{\varepsilon}\right)$ instead of $\phi\left(F_{\varepsilon}\right)$, we obtain the desired asymptotic expansion.

At the end of this subsection, we gather well-known facts about SDE (2.1) and ODE (2.2) for later use. The Jacobian $J_{t}$ of $\phi(t, x, h)$ with respect to $x$ and its inverse $K_{t}$ satisfy the following ODEs:

$$
\begin{gathered}
d J_{t}=\sum_{i=1}^{r} \nabla V_{i}\left(\phi_{t}\right) J_{t} d h_{t}^{i} \quad \text { with } J_{0}=\mathrm{Id}_{d}, \\
d K_{t}=-\sum_{i=1}^{r} K_{t} \nabla V_{i}\left(\phi_{t}\right) d h_{t}^{i} \quad \text { with } K_{0}=\mathrm{Id}_{d} .
\end{gathered}
$$

Here, $J, K, \nabla V_{i}$ are all $d \times d$ matrices. Note that $K_{t}=J_{t}^{-1}$. When the dependence on $h$ and $x$ needs to be specified, we write $J_{t}(h)$ or $J(t, x, h)$, and so forth. The deterministic Malliavin covariance matrix is given by

$$
\sigma\left[\phi_{1}\right](h)=J_{1}(h)\left\{\int_{0}^{1} J_{t}(h)^{-1} \mathbf{V}\left(\phi_{t}(h)\right) \mathbf{V}\left(\phi_{t}(h)\right)^{*}\left(J_{t}(h)^{-1}\right)^{*} d t\right\} J_{1}(h)^{*}
$$

and $\sigma\left[\psi_{1}\right](h)=\Pi_{\mathcal{V}} \sigma\left[\phi_{1}\right](h) \Pi_{\mathcal{V}}^{*}$, where we set $\mathbf{V}:=\left[V_{1}, \ldots, V_{r}\right] \in \operatorname{Mat}(d, r)$ for simplicity.

Similarly, the Jacobian process $J_{t}^{\varepsilon}$ for SDE (2.1) and its inverse $K_{t}^{\varepsilon}$ satisfy the following SDEs:

$$
d J_{t}^{\varepsilon}=\varepsilon \sum_{i=1}^{r} \nabla V_{i}\left(X_{t}^{\varepsilon}\right) J_{t}^{\varepsilon} \circ d w_{t}^{i}+\varepsilon^{2} \nabla V_{0}\left(X_{t}^{\varepsilon}\right) J_{t}^{\varepsilon} d t \quad \text { with } J_{0}^{\varepsilon}=\operatorname{Id}_{d},
$$




$$
d K_{t}^{\varepsilon}=-\varepsilon \sum_{i=1}^{r} K_{t}^{\varepsilon} \nabla V_{i}\left(X_{t}^{\varepsilon}\right) \circ d w_{t}^{i}-\varepsilon^{2} K_{t}^{\varepsilon} \nabla V_{0}\left(X_{t}^{\varepsilon}\right) d t \quad \text { with } K_{0}^{\varepsilon}=\operatorname{Id}_{d}
$$

As before, $K_{t}^{\varepsilon}=\left(J_{t}^{\varepsilon}\right)^{-1}$. When necessary we write $J_{t}^{\varepsilon}(w)$ or $J^{\varepsilon}(t, x, w)$, and so forth. The Malliavin covariance matrix is given by

$$
\varepsilon^{-2} \sigma\left[X_{1}^{\varepsilon}\right]=J_{1}^{\varepsilon}\left\{\int_{0}^{1}\left(J_{t}^{\varepsilon}\right)^{-1} \mathbf{V}\left(X_{t}^{\varepsilon}\right) \mathbf{V}\left(X_{t}^{\varepsilon}\right)^{*}\left(J_{t}^{\varepsilon}\right)^{-1, *} d t\right\}\left(J_{1}^{\varepsilon}\right)^{*}
$$

and $\sigma\left[Y_{t}^{\varepsilon}\right](w)=\Pi_{\mathcal{V}} \sigma\left[X_{t}^{\varepsilon}\right](w) \Pi_{\mathcal{V}}^{*}$ a.s. (The dependence on $w$ is suppressed above.)

3.2. Preliminaries from rough path theory. In this subsection we recall the geometric rough path space with Hölder or Besov norm and quasisure property of rough path lift. For basic properties of geometric rough path space, we refer to Lyons and Qian [35], Lyons et al. [34], and Friz and Victoir [16]. For the geometric rough path space with Besov norm, we refer to [16, Appendix A.2]. Quasisure property of rough path lift is summarized in Inahama [25].

We assume that the Besov parameters $(\alpha, 4 m)$ satisfy the following condition:

$$
\frac{1}{3}<\alpha<\frac{1}{2}, \quad m=1,2,3, \ldots, \quad \alpha-\frac{1}{4 m}>\frac{1}{3}, \quad \text { and } \quad 4 m\left(\frac{1}{2}-\alpha\right)>1 .
$$

We choose such a pair $(\alpha, 4 m)$ and fix it throughout this paper.

We denote by $G \Omega_{\alpha}^{H}\left(\mathbb{R}^{r}\right), 1 / 3<\alpha<1 / 2$, the geometric rough path space over $\mathbb{R}^{d}$ with $\alpha$-Hölder norm. Let $C_{0}^{\beta-H}([0,1], \mathbb{R}), 0<\beta \leqslant 1$, be the Banach space of all the $\mathbb{R}$-valued, $\beta$-Hölder continuous paths that start at 0 . If $\alpha+\beta>1$, then the Young pairing

$$
G \Omega_{\alpha}^{H}\left(\mathbb{R}^{r}\right) \times C_{0}^{\beta-H}([0,1], \mathbb{R}) \ni(\mathbf{w}, \lambda) \mapsto(\mathbf{w}, \lambda) \in G \Omega_{\alpha}^{H}\left(\mathbb{R}^{r+1}\right)
$$

is a well-defined, locally Lipschitz continuous map. (See [16, Section 9.4] for instance.)

Now we consider a system of RDEs driven by the Young pairing $(\mathbf{w}, \lambda) \in$ $G \Omega_{\alpha}^{H}\left(\mathbb{R}^{d+1}\right)$ of $\mathbf{w} \in G \Omega_{\alpha}^{H}\left(\mathbb{R}^{d}\right)$ and $\lambda \in C_{0}^{1-H}\left([0,1], \mathbb{R}^{1}\right)$. (In most cases, we assume $\lambda_{t}=$ const $\times t$. $)$ For vector fields $V_{i}: \mathbb{R}^{d} \rightarrow \mathbb{R}^{d}(0 \leqslant i \leqslant r)$, consider

$$
d x_{t}=\sum_{i=1}^{r} V_{i}\left(x_{t}\right) d w_{t}^{i}+V_{0}\left(x_{t}\right) d \lambda_{t} \quad \text { with } x_{0}=x \in \mathbb{R}^{d} \text {. }
$$

The RDEs for the Jacobian process and its inverse are given as follows;

$$
d J_{t}=\sum_{i=1}^{r} \nabla V_{i}\left(x_{t}\right) J_{t} d w_{t}^{i}+\nabla V_{0}\left(x_{t}\right) J_{t} d \lambda_{t} \quad \text { with } J_{0}=\operatorname{Id}_{d} \in \operatorname{Mat}(d, d),
$$




$$
d K_{t}=-\sum_{i=1}^{r} K_{t} \nabla V_{i}\left(x_{t}\right) d w_{t}^{i}-K_{t} \nabla V_{0}\left(x_{t}\right) d \lambda_{t} \quad \text { with } K_{0}=\operatorname{Id}_{d} \in \operatorname{Mat}(d, d) .
$$

Here, $J, K$, and $\nabla V_{i}$ are all Mat $(d, d)$-valued.

Assume that $V_{i}$ 's are of $C_{b}^{4}$ for a while. Then, a global solution of (3.17)-(3.19) exists for any $\mathbf{w}$ and $\lambda$. Moreover, Lyons' continuity theorem holds. (The linear growth case is complicated and will be discussed later.) In that case, the following maps, where $(\mathbf{w}, \lambda) \in G \Omega_{\alpha}^{H}\left(\mathbb{R}^{r}\right) \times C_{0}^{1-H}\left([0,1], \mathbb{R}^{1}\right)$, are continuous:

$$
\begin{aligned}
(\mathbf{w}, \lambda) & \mapsto(\mathbf{w}, \lambda) \in G \Omega_{\alpha}^{H}\left(\mathbb{R}^{r+1}\right) \\
& \mapsto(\mathbf{w}, \lambda ; \mathbf{x}, \mathbf{J}, \mathbf{K}) \in G \Omega_{\alpha}^{H}\left(\mathbb{R}^{r+1} \oplus \mathbb{R}^{d} \oplus \operatorname{Mat}(d, d)^{\oplus 2}\right) \\
& \mapsto(\mathbf{x}, \mathbf{J}, \mathbf{K}) \in G \Omega_{\alpha}^{H}\left(\mathbb{R}^{d} \oplus \operatorname{Mat}(d, d)^{\oplus 2}\right) .
\end{aligned}
$$

Here, the first map is the Young pairing, the second is the Lyons-Itô map, and the third is the canonical projection. (The map $(\mathbf{w}, \lambda) \mapsto \mathbf{x}$ will be denoted by $\Phi: G \Omega_{\alpha}^{H}\left(\mathbb{R}^{r}\right) \times C_{0}^{1-H}([0,1], \mathbb{R}) \rightarrow G \Omega_{\alpha}^{H}\left(\mathbb{R}^{d}\right)$.) Recall that in Lyons' formulation of rough path theory, the initial values of the first level paths must be adjusted. Note that $\left(\mathrm{Id}+\mathbf{J}_{0, t}^{1}\right)^{-1}=\mathrm{Id}+\mathbf{K}_{0, t}^{1}$ always holds.

When $\mathbf{w}$ is the natural lift $h \in \mathcal{H}$ and $\lambda \equiv 0$, the first level path

$$
t \mapsto\left(x+\mathbf{x}_{0, t}^{1}, \mathrm{Id}+\mathbf{J}_{0, t}^{1}, \mathrm{Id}+\mathbf{K}_{0, t}^{1}\right)
$$

is identical to the solution of a system (2.2), (3.10), (3.11). Here, $\mathbf{x}_{0, t}^{1}$ is the first level path of $\mathbf{x}$ evaluated at $(0, t)$, and so forth. Similarly, if $(\mathbf{w}, \lambda)=\left(\varepsilon \mathbf{W}, \lambda^{\varepsilon}\right)$, where $\mathbf{W}=\mathcal{L}(w)$ is the Brownian rough path under $\mu$ and $\lambda_{t}^{\varepsilon}=\varepsilon^{2} t$, then (3.21) coincides with the solution of (2.1), (3.13), (3.14) a.s.

We define a continuous function $\Gamma: G \Omega_{\alpha}^{H}\left(\mathbb{R}^{r}\right) \times C_{0}^{1-H}([0,1], \mathbb{R}) \rightarrow \operatorname{Mat}(d, d)$ as follows: set

$$
\Gamma(\mathbf{w}, \lambda)=\left(\operatorname{Id}+\mathbf{J}_{0, t}^{1}\right) \hat{\Gamma}(\mathbf{w}, \lambda)\left(\operatorname{Id}+\mathbf{J}_{0, t}^{1}\right)^{*},
$$

where

$$
\hat{\Gamma}(\mathbf{w}, \lambda):=\int_{0}^{1}\left(\operatorname{Id}+\mathbf{K}_{0, t}^{1}\right) \mathbf{V}\left(x+\mathbf{x}_{0, t}^{1}\right) \mathbf{V}\left(x+\mathbf{x}_{0, t}^{1}\right)^{*}\left(\operatorname{Id}+\mathbf{K}_{0, t}^{1}\right)^{*} d t
$$

with $\mathbf{V}:=\left[V_{1}, \ldots, V_{r}\right] \in \operatorname{Mat}(d, r)$.

From (3.12) and (3.15) we can easily see the following: if $\lambda_{t}^{\varepsilon}=\varepsilon^{2} t$, then $\Gamma\left(\varepsilon \mathbf{W}, \lambda^{\varepsilon}\right)=\varepsilon^{-2} \sigma\left[X_{1}^{\varepsilon}\right](w)$ for $\mu$-almost all $w$, where $X_{1}^{\varepsilon}$ denotes the solution of SDE (2.1) at $t=1$. If $\lambda_{t} \equiv 0$ and $\mathbf{h}=\mathcal{L}(h)$ is the natural lift of $h \in \mathcal{H}$, then $\Gamma(\mathbf{h}, 0)=\sigma\left[\phi_{1}\right](h)$, the deterministic Malliavin covariance matrix given in (3.12). From these we can easily see that $\Pi_{\mathcal{V}} \Gamma\left(\varepsilon \mathbf{w}, \lambda^{\varepsilon}\right) \Pi_{\mathcal{V}}^{*}=\varepsilon^{-2} \sigma\left[Y_{1}^{\varepsilon}\right](w)$ a.s. and $\Pi_{\mathcal{V}} \Gamma(\mathbf{h}, 0) \Pi_{\mathcal{V}}^{*}=\sigma\left[\psi_{1}\right](h)$. 
REMARK 3.3. In this paper we use Lyons' continuity theorem only with respect to $\alpha$-Hölder topology $(1 / 3<\alpha<1 / 2)$ and for $C_{b}^{4}$-coefficient vector fields. We do not try to extend it to the case of unbounded coefficient vector fields or Besov topology.

Now we introduce the Besov topology on the rough path space. For $(\alpha, 4 m)$ which satisfies (3.16), $G \Omega_{\alpha, 4 m}^{B}\left(\mathbb{R}^{r}\right)$ denotes the geometric rough path space over $\mathbb{R}^{r}$ with $(\alpha, 4 m)$-Besov norm. Recall that the distance on this space is given by

$$
\begin{aligned}
d(\mathbf{w}, \hat{\mathbf{w}})= & \left\|\mathbf{w}^{1}-\hat{\mathbf{w}}^{1}\right\|_{\alpha, 4 m-B}+\left\|\mathbf{w}^{2}-\hat{\mathbf{w}}^{2}\right\|_{2 \alpha, 2 m-B} \\
:= & \left(\iint_{0 \leqslant s<t \leqslant 1} \frac{\left|\mathbf{w}_{s, t}^{1}-\hat{\mathbf{w}}_{s, t}^{1}\right|^{4 m}}{|t-s|^{1+4 m \alpha}} d s d t\right)^{1 / 4 m} \\
& +\left(\iint_{0 \leqslant s<t \leqslant 1} \frac{\left|\mathbf{w}_{s, t}^{2}-\hat{\mathbf{w}}_{s, t}^{2}\right|^{2 m}}{|t-s|^{1+4 m \alpha}} d s d t\right)^{1 / 2 m} .
\end{aligned}
$$

By the Besov-Hölder embedding theorem for rough path spaces, there is a continuous embedding $G \Omega_{\alpha, 4 m}^{B}\left(\mathbb{R}^{r}\right) \hookrightarrow G \Omega_{\alpha-(1 / 4 m)}^{H}\left(\mathbb{R}^{r}\right)$. If $\alpha<\alpha^{\prime}<1 / 2$, there is a continuous embedding $G \Omega_{\alpha^{\prime}}^{H}\left(\mathbb{R}^{r}\right) \hookrightarrow G \Omega_{\alpha, 4 m}^{B}\left(\mathbb{R}^{r}\right)$. We remark that we will not write these embeddings explicitly. (For example, if we write $\Phi(\mathbf{w}, \lambda)$ for $(\mathbf{w}, \lambda) \in G \Omega_{\alpha, 4 m}^{B}\left(\mathbb{R}^{r}\right) \times C_{0}^{1-H}([0,1], \mathbb{R})$, then it is actually the composition of the first embedding map above and $\Phi$ with respect to $\{\alpha-1 /(4 m)\}$-Hölder topology.)

Note also that the Young translation by $h \in \mathcal{H}$ works perfectly on $G \Omega_{\alpha, 4 m}^{B}\left(\mathbb{R}^{r}\right)$ under (3.16). The map $(\mathbf{w}, h) \mapsto \tau_{h}(\mathbf{w})$ is continuous from $G \Omega_{\alpha, 4 m}^{B}\left(\mathbb{R}^{r}\right) \times \mathcal{H}$ to $G \Omega_{\alpha, 4 m}^{B}\left(\mathbb{R}^{r}\right)$, where $\tau_{h}(\mathbf{w})$ is called the Young translation of $\mathbf{w}$ by $h$ and is defined by

$$
\tau_{h}(\mathbf{w})_{s, t}^{1}:=\mathbf{w}_{s, t}^{1}+\mathbf{h}_{s, t}^{1}, \quad \tau_{h}(\mathbf{w})_{s, t}^{2}:=\mathbf{w}_{s, t}^{2}+\mathbf{h}_{s, t}^{2}+\int_{s}^{t} \mathbf{w}_{s, u}^{1} d h_{u}+\int_{s}^{t} \mathbf{h}_{s, u}^{1} d w_{u} .
$$

Here, the integrals are in the Young (or Riemann-Stieltjes) sense and we set $w_{t}:=$ $\mathbf{w}_{0, t}^{1}$. Moreover, there exists a positive constant $C=C_{\alpha, 4 m}$ such that

$$
\begin{gathered}
\left\|\tau_{h}(\mathbf{w})^{1}\right\|_{\alpha, 4 m-B} \leqslant\left\|\mathbf{w}^{1}\right\|_{\alpha, 4 m-B}+C\|h\|_{\mathcal{H}}, \\
\left\|\tau_{h}(\mathbf{w})^{2}\right\|_{2 \alpha, 2 m-B} \leqslant\left\|\mathbf{w}^{2}\right\|_{2 \alpha, 2 m-B}+2 C\left\|\mathbf{w}^{1}\right\|_{\alpha, 4 m-B}\|h\|_{\mathcal{H}}+C^{2}\|h\|_{\mathcal{H}}^{2}
\end{gathered}
$$

hold for all $h \in \mathcal{H}$ and $\mathbf{w} \in G \Omega_{\alpha, 4 m}^{B}\left(\mathbb{R}^{r}\right)$.

For $\gamma>0$ and $h \in \mathcal{H}$, we set

$$
\begin{aligned}
U_{h, \gamma} & =\left\{\mathbf{w} \in G \Omega_{\alpha, 4 m}^{B}\left(\mathbb{R}^{r}\right) \mid\left\|\tau_{-h}(\mathbf{w})^{1}\right\|_{\alpha, 4 m-B}^{4 m}+\left\|\tau_{-h}(\mathbf{w})^{2}\right\|_{2 \alpha, 2 m-B}^{2 m}<\gamma^{4 m}\right\}, \\
U_{h, \gamma}^{\prime} & =U_{h, 2^{-1 / 4 m} \gamma} \\
& =\left\{\mathbf{w} \in G \Omega_{\alpha, 4 m}^{B}\left(\mathbb{R}^{r}\right) \mid\left\|\tau_{-h}(\mathbf{w})^{1}\right\|_{\alpha, 4 m-B}^{4 m}+\left\|\tau_{-h}(\mathbf{w})^{2}\right\|_{2 \alpha, 2 m-B}^{2 m}<\gamma^{4 m} / 2\right\} .
\end{aligned}
$$


When $h=0$ we simply write $U_{\gamma}$ and $U_{\gamma}^{\prime}$. By the continuity of $\tau_{h}$ with respect to the $(\alpha, 4 m)$-Besov rough path topology, $\left\{U_{h, \gamma}\right\}_{\gamma>0}$ forms a system of open neighborhoods around $\mathbf{h}=\mathcal{L}(h)$ and so does $\left\{U_{h, \gamma}^{\prime}\right\}_{\gamma>0}$. Clearly, $U_{h, \gamma}^{\prime}=U_{h, \gamma 2^{-1 / 4 m}}$, $U_{h, \gamma}=\tau_{h}\left(U_{\gamma}\right)$ and $U_{h, \gamma}^{\prime}=\tau_{h}\left(U_{\gamma}^{\prime}\right)$.

Now we discuss quasisure properties of rough path lift map $\mathcal{L}$ from $\mathcal{W}$ to $G \Omega_{\alpha, 4 m}^{B}\left(\mathbb{R}^{r}\right)$. For $k=1,2, \ldots$ and $w \in \mathcal{W}$, we denote by $w(k)$ the $k$ th dyadic piecewise linear approximation of $w$ associated with the partition $\left\{l 2^{-k} \mid 0 \leqslant l\right.$ $\left.\leqslant 2^{k}\right\}$ of $[0,1]$. We set

$$
\mathcal{Z}_{\alpha, 4 m}:=\left\{w \in \mathcal{W} \mid\{\mathcal{L}(w(k))\}_{k=1}^{\infty} \text { is Cauchy in } G \Omega_{\alpha, 4 m}^{B}\left(\mathbb{R}^{r}\right)\right\}
$$

We define $\mathcal{L}: \mathcal{W} \rightarrow G \Omega_{\alpha, 4 m}^{B}\left(\mathbb{R}^{r}\right)$ by $\mathcal{L}(w)=\lim _{m \rightarrow \infty} \mathcal{L}(w(k))$ if $w \in \mathcal{Z}_{\alpha, 4 m}$ and we do not define $\mathcal{L}(w)$ if $w \notin \mathcal{Z}_{\alpha, 4 m}$. (We always use this version of $\mathcal{L}$.) Note that $\mathcal{H}$ and $C_{0}^{\beta-H}\left([0,1], \mathbb{R}^{r}\right)$ with $\beta \in(1 / 2,1]$ are subsets of $\mathcal{Z}_{\alpha, 4 m}$ and this lift for elements of such subsets coincides with the direct lift by means of the RiemannStieltjes integral. Under scalar multiplication and Cameron-Martin translation, $\mathcal{Z}_{\alpha, 4 m}$ is invariant. Moreover, $c \mathcal{L}(w)=\mathcal{L}(c w)$ and $\tau_{h}(\mathcal{L}(w))=\mathcal{L}(w+h)$ for any $w \in \mathcal{Z}_{\alpha, 4 m}, c \in \mathbb{R}$, and $h \in \mathcal{H}$.

It is known that $\mathcal{Z}_{\alpha, 4 m}^{c}$ is slim, that is the $(p, r)$-capacity of this set is zero for any $p \in(1, \infty)$ and $r \in \mathbb{N}$. (See Aida [1], Inahama [23, 25].) Therefore, from a viewpoint of quasisure analysis, the lift map $\mathcal{L}$ is well defined. Moreover, the map $\mathcal{W} \ni w \mapsto \mathcal{L}(w) \in G \Omega_{\alpha, 4 m}^{B}\left(\mathbb{R}^{r}\right)$ is $\infty$-quasicontinuous (Aida [1]). We often write $\mathbf{W}:=\mathcal{L}(w)$ when it is regarded as a rough path space-valued random variable defined on $\mathcal{W}$. Due to Lyons' continuity theorem and uniqueness of quasicontinuous modification, $\tilde{X}^{\varepsilon}(\cdot, x, w)=x+\Phi\left(\varepsilon \mathcal{L}(w), \varepsilon^{2} \lambda\right)^{1}$ holds quasisurely if $V_{i}(0 \leqslant i \leqslant d)$ is of $C_{b}^{3}$. (Here, $\lambda_{t}=t$ and $\tilde{X}^{\varepsilon}(\cdot, x, w)$ denotes the $\infty$-quasicontinuous modification of the path space-valued random variable $w \mapsto X^{\varepsilon}(\cdot, x, w)$.) A similar remark holds for $J^{\varepsilon}$ and $K^{\varepsilon}$.

Before closing this subsection, we give a remark for the coefficient vector fields with linear growth.

REMARK 3.4. If $V_{i}(0 \leqslant i \leqslant d)$ satisfies (A1) and has linear growth, it is not easy to prove the existence of a global solution of $\operatorname{RDE}(3.17)$ for a given $(\mathbf{w}, \lambda) \in$ $G \Omega_{\alpha}^{H}\left(\mathbb{R}^{r}\right) \times C_{0}^{1-H}([0,1], \mathbb{R})$. However, Bailleul [2] recently proved it. Hence, the Lyons-Itô map $\Phi$ can be defined on the whole space $G \Omega_{\alpha}^{H}\left(\mathbb{R}^{r}\right) \times C_{0}^{1-H}([0,1], \mathbb{R})$ and Lyons' continuity theorem holds under (A1), too. However, almost no other properties of the solution are known under (A1) at this moment. Therefore, we basically assume that $V_{i}(0 \leqslant i \leqslant r)$ is of $C_{b}^{\infty}$ when we use RDEs. The reason why we may do so is as follows. In the proof of our main theorem we discard contributions from rough paths distant from $\mathcal{L}\left(\mathcal{K}_{a}^{\min }\right)$ by using large deviation 
theory. Therefore, by simple cut-off argument, the problem reduces to the $C_{b}^{\infty}$ case anyway.

\subsection{Some useful results in Malliavin calculus on rough path space. First,} we recall Taylor-like expansion of (Lyons-)Itô map. In the proof of our main theorem, we compute the Cameron-Martin translation of $X^{\varepsilon}$. For a CameronMartin path $h \in \mathcal{H}$, we set $X_{t}^{\varepsilon, h}:=X^{\varepsilon}(t, x, w+(h / \varepsilon))$, which satisfies

$$
d X_{t}^{\varepsilon, h}=\sum_{i=1}^{r} V_{i}\left(X_{t}^{\varepsilon, h}\right) \circ\left(\varepsilon d w_{t}^{i}+d h_{t}^{i}\right)+\varepsilon^{2} V_{0}\left(X_{t}^{\varepsilon, h}\right) d t \quad \text { with } X_{0}^{\varepsilon, h}=x \in \mathbb{R}^{d} .
$$

Similarly, the translations of the Jacobian process and its inverse satisfy:

$$
\begin{aligned}
& d J_{t}^{\varepsilon, h}=\sum_{i=1}^{r} \nabla V_{i}\left(X_{t}^{\varepsilon, h}\right) J_{t}^{\varepsilon, h} \circ\left(\varepsilon d w_{t}^{i}+d h_{t}^{i}\right)+\varepsilon^{2} \nabla V_{0}\left(X_{t}^{\varepsilon, h}\right) J_{t}^{\varepsilon, h} d t \\
& \text { with } J_{0}^{\varepsilon, h}=\operatorname{Id}_{d}
\end{aligned}
$$

and

$$
\begin{aligned}
& d K_{t}^{\varepsilon, h}=-\sum_{i=1}^{r} K_{t}^{\varepsilon, h} \nabla V_{i}\left(X_{t}^{\varepsilon, h}\right) \circ\left(\varepsilon d w_{t}^{i}+d h_{t}^{i}\right)-\varepsilon^{2} K_{t}^{\varepsilon, h} \nabla V_{0}\left(X_{t}^{\varepsilon, h}\right) d t \\
& \text { with } K_{0}^{\varepsilon, h}=\operatorname{Id}_{d} .
\end{aligned}
$$

It is easy to see that $\left(X^{\varepsilon, h}, J^{\varepsilon, h}, K^{\varepsilon, h}\right)$ coincides a.s. with (3.21) with the driving rough path being $\left(\tau_{h}(\varepsilon \mathbf{W}), \lambda^{\varepsilon}\right)=\left(\varepsilon \mathcal{L}(w+h / \varepsilon), \lambda^{\varepsilon}\right)$.

It is known that under (A1) the following asymptotic expansion holds in $\mathbb{D}_{\infty}\left(\mathbb{R}^{d}\right)$ as $\varepsilon \searrow 0$ :

$$
X^{\varepsilon}\left(1, x, w+\frac{h}{\varepsilon}\right)=f_{0}(h)+\varepsilon f_{1}(w ; h)+\cdots+\varepsilon^{k} f_{k}(w ; h)+Q_{k+1}^{\varepsilon}(w ; h)
$$

where $Q_{k+1}^{\varepsilon}(w ; h)=O\left(\varepsilon^{k+1}\right)$. Note that $f_{0}(h)=\phi_{1}(h)$. It immediately follows that

$$
Y^{\varepsilon}\left(1, x, w+\frac{h}{\varepsilon}\right)=g_{0}(h)+\varepsilon g_{1}(w ; h)+\cdots+\varepsilon^{k} g_{k}(w ; h)+R_{k+1}^{\varepsilon}(w ; h)
$$

in $\mathbb{D}_{\infty}\left(\mathbb{R}^{d}\right)$ as $\varepsilon \searrow 0$, where we set $R_{k+1}^{\varepsilon}(w ; h)=\Pi_{\mathcal{V}}\left(Q_{k+1}^{\varepsilon}(w ; h)\right)$ and $g_{j}(w ; h)=\Pi_{\mathcal{V}}\left(f_{j}(w ; h)\right)$ for all $j \geqslant 0$. Note that $g_{0}(h)=\psi_{1}(h)$ and $g_{1}(w ; h)=$ $D \psi_{1}(h)\langle w\rangle$. Moreover, these asymptotic expansions are uniform in $h$ as $h$ varies in an arbitrary bounded set in $\mathcal{H}$. 
The expansion (3.29) has a counterpart in rough path theory, which we call Taylor-like expansion of the Lyons-Itô map $\Phi$. (See Inahama and Kawabi [28] and Inahama [24].) Assume $1 / 3<\alpha<1 / 2$ and that $V_{i}$ is of $C_{b}^{\infty}$ for a while $(0 \leqslant i \leqslant r)$. Then, there exist continuous maps $\hat{f}_{k}: G \Omega_{\alpha}^{H}\left(\mathbb{R}^{r}\right) \times \mathcal{H} \mapsto \mathbb{R}^{d}$ and $\hat{Q}_{k+1}^{\varepsilon}: G \Omega_{\alpha}^{H}\left(\mathbb{R}^{r}\right) \times \mathcal{H} \mapsto \mathbb{R}^{d}$ for $k=0,1, \ldots$ which satisfy the following (i) and (ii):

(i) For all $k=0,1, \ldots$ and $\varepsilon \in[0,1]$,

$$
f_{k}(w ; h)=\hat{f}_{k}(\mathbf{W} ; h) \quad \text { and } \quad Q_{k+1}^{\varepsilon}(w ; h)=\hat{Q}_{k+1}^{\varepsilon}(\mathbf{W} ; h), \quad \mu \text {-a.s., }(3.31)
$$

where we set

$$
\hat{Q}_{k+1}^{\varepsilon}(\mathbf{w} ; h):=x+\Phi\left(\tau_{h}(\varepsilon \mathbf{w}), \lambda^{\varepsilon}\right)_{0,1}^{1}-\left\{\hat{f}_{0}(h)+\varepsilon \hat{f}_{1}(\mathbf{w} ; h)+\cdots+\varepsilon^{k} \hat{f}_{k}(\mathbf{w} ; h)\right\} .
$$

(ii) For any $k \in \mathbb{N}, \rho>0, h \in \mathcal{H}$, there exist positive constants $C_{k}$ and $C_{k, \rho}^{\prime}$ such that

$$
\left|\hat{f}_{k}(\mathbf{w} ; h)\right| \leqslant C_{k}\left(1+\left\|\mathbf{w}^{1}\right\|_{\alpha-H}+\left\|\mathbf{w}^{2}\right\|_{2 \alpha-H}^{1 / 2}\right)^{k}
$$

for any $\mathbf{w} \in G \Omega_{\alpha}^{H}\left(\mathbb{R}^{r}\right)$ and

$$
\left|\hat{Q}_{k+1}^{\varepsilon}(\mathbf{w} ; h)\right| \leqslant C_{k, \rho}^{\prime}\left(\varepsilon+\left\|(\varepsilon \mathbf{w})^{1}\right\|_{\alpha-H}+\left\|(\varepsilon \mathbf{w})^{2}\right\|_{2 \alpha-H}^{1 / 2}\right)^{k+1}
$$

if $\left\|(\varepsilon \mathbf{w})^{1}\right\|_{\alpha-H}+\left\|(\varepsilon \mathbf{w})^{2}\right\|^{1 / 2} \leqslant \rho$. Of course, $C_{k}$ and $C_{k, \rho}^{\prime}$ also depend on $h$, but in fact they depend only on $\|h\|_{\mathcal{H}}$.

Note that the inequalities (3.33) and (3.34) are deterministic. It is obvious that $\hat{g}_{k}(w ; h):=\Pi_{\mathcal{V}}\left(\hat{f}_{k}(w ; h)\right)$ and $\hat{R}_{k+1}^{\varepsilon}(\mathbf{w} ; h):=\Pi_{\mathcal{V}}\left(\hat{Q}_{k+1}^{\varepsilon}(\mathbf{w} ; h)\right)$ have similar properties to (3.31)-(3.34).

REMARK 3.5. (i) Heuristically, (3.31) means that, in a sense, $\hat{f}_{k}$ and $\hat{Q}_{k+1}^{\varepsilon}$ are 'lifts' of $f_{k}$ and $Q_{k+1}^{\varepsilon}$, respectively.

(ii) By abusing notations, we simply write $f_{k}, g_{k}, Q_{k+1}^{\varepsilon}, R_{k+1}^{\varepsilon}$ for $\hat{f}_{k}, \hat{g}_{k}, \hat{Q}_{k+1}^{\varepsilon}$, $\hat{R}_{k+1}^{\varepsilon}$ in the sequel.

(iii) If we assume that $V_{i}$ is of $C_{b}^{\infty}(0 \leqslant i \leqslant r)$, then we can actually obtain (3.29) via the deterministic expansion (3.31)-(3.34) on the geometric rough path space. (See Inahama [26].) Under (A1), however, it is not known yet whether this is possible or not. 
(iv) There is a simple expression of $\hat{f}_{k}(\mathbf{w}, h)$ when the drift vector field $V_{0} \equiv 0$. Suppose that $\xi_{l} \in \mathcal{H}(l=1,2, \ldots)$ converges to $\mathbf{w}$ in $G \Omega_{\alpha}^{H}\left(\mathbb{R}^{r}\right)$. Then,

$$
\hat{f}_{k}(\mathbf{w}, h)=\lim _{l \rightarrow \infty} \frac{1}{k !} D^{k} \phi_{1}(h)\left\langle\xi_{l}, \ldots, \xi_{l}\right\rangle, \quad(k \text {-times }) .
$$

Here, $D$ stands for the Fréchet derivative on $\mathcal{H}$. (Even when $V_{0}$ does not vanish, this kind of expression exists. Since it looks quite complicated due the factor $\varepsilon^{2}$ in front of $V_{0}$, we omit it, however.)

In order for $F\left(X^{\varepsilon}(1, x, w+h / \varepsilon)\right)$ to admit expansions as above, $F$ need not be a projection or a linear map. In the next lemma, we show that for quite general $F$, it admits expansions in both senses. The proof is straightforward. So we omit it. We also remark that the expansions in Lemma 3.6 are uniform in $h$ as $h$ varies in any bounded set in $\mathcal{H}$. (Lemma 3.6 will be used in the proof of the manifold case, not in the Euclidean case.)

LEMMA 3.6. Let $F: \mathbb{R}^{d} \rightarrow \mathbb{R}^{e}$ be a smooth map such that $\nabla^{j} F$ is bounded for all $j \geqslant 1$. Then, we have the following (i)-(ii):

(i) As $\varepsilon \searrow 0$,

$$
F\left(X^{\varepsilon}\left(1, x, w+\frac{h}{\varepsilon}\right)\right)=g_{0}^{F}(h)+\varepsilon g_{1}^{F}(w ; h)+\cdots+\varepsilon^{k} g_{k}^{F}(w ; h)+R_{k+1}^{\varepsilon, F}(w ; h)
$$

in $\mathbb{D}_{\infty}\left(\mathbb{R}^{d}\right)$. Here, $R_{k+1}^{\varepsilon, F}(w ; h)=O\left(\varepsilon^{k+1}\right)$ in $\mathbb{D}_{\infty}\left(\mathbb{R}^{d}\right)$ and $g_{j}^{F}(w ; h)$ are determined by the formal composition of (3.29) and the Taylor expansion of F. For example,

$$
\begin{gathered}
g_{0}^{F}(h)=F\left(f_{0}(h)\right), \\
g_{1}^{F}(w ; h)=\nabla F\left(f_{0}(h)\right)\left\langle f_{1}(w ; h)\right\rangle, \\
g_{2}^{F}(w ; h)=\nabla F\left(f_{0}(h)\right)\left\langle f_{2}(w ; h)\right\rangle+\frac{1}{2} \nabla^{2} F\left(f_{0}(h)\right)\left\langle f_{1}(w ; h), f_{1}(w ; h)\right\rangle
\end{gathered}
$$

and so on.

(ii) Similarly, if $\hat{g}_{j}^{F}(\mathbf{w} ; h)$ is determined by the formal composition of (3.32) and the Taylor expansion of $F$, then we have

$$
F\left(x+\Phi\left(\tau_{h}(\varepsilon \mathbf{w}), \lambda^{\varepsilon}\right)_{0,1}^{1}\right)=\hat{g}_{0}^{F}(h)+\varepsilon \hat{g}_{1}^{F}(\mathbf{w} ; h)+\cdots+\varepsilon^{k} \hat{g}_{k}^{F}(\mathbf{w} ; h)+\hat{R}_{k+1}^{\varepsilon, F}(\mathbf{w} ; h),
$$

where $\hat{g}_{k}^{F}$ and $\hat{R}_{k+1}^{\varepsilon, F}$ satisfy essentially the same estimates as in (3.33) and (3.34) (for different positive constants $C_{k}$ and $C_{k, \rho}^{\prime}$ ). Moreover, we have $\hat{g}_{j}^{F}(\mathbf{W} ; h)=g_{j}^{F}(w ; h)$ and $R_{k+1}^{\varepsilon, F}(w ; h)=\hat{R}_{k+1}^{\varepsilon, F}(\mathbf{W} ; h), \mu$-a.s. 
Next we give a simple lemma for Malliavin covariance matrices of $X^{\varepsilon}(1, x$, $w+h / \varepsilon)$ and $Y^{\varepsilon}(1, x, w+h / \varepsilon)$. For any $h \in \mathcal{H}$, the Malliavin covariance matrix of $Q_{1}^{\varepsilon}(w ; h) / \varepsilon=\left\{X^{\varepsilon}(1, x, w+h / \varepsilon)-f_{0}(h)\right\} / \varepsilon$ is given by

$$
\begin{aligned}
\sigma\left[Q_{1}^{\varepsilon}(\cdot ; h) / \varepsilon\right](w) & =\int_{0}^{1} J_{1}^{\varepsilon, h} K_{t}^{\varepsilon, h} \mathbf{V}\left(X_{t}^{\varepsilon, h}\right) \mathbf{V}\left(X_{t}^{\varepsilon, h}\right)^{*}\left(K_{t}^{\varepsilon, h}\right)^{*}\left(J_{1}^{\varepsilon, h}\right)^{*} d t \\
& =\Gamma\left(\tau_{h}(\varepsilon \mathbf{W}), \lambda^{\varepsilon}\right),
\end{aligned}
$$

where $\lambda_{t}^{\varepsilon}=\varepsilon^{2} t, \mathbf{W}=\mathcal{L}(w)$ is Brownian rough path and $\Gamma$ is defined in (3.22). Note that $\sigma\left[R_{1}^{\varepsilon}(\cdot ; h) / \varepsilon\right](w)=\Pi_{\mathcal{V}} \sigma\left[Q_{1}^{\varepsilon}(\cdot ; h) / \varepsilon\right](w) \Pi_{\mathcal{V}}^{*}$.

Let $h \in \mathcal{K}_{a}^{\min }$ and let $\left\{e_{i}\right\}_{i=1}^{n^{\prime}}$ be an orthonormal basis of $T_{h} \mathcal{K}_{a}^{\min } \subset \mathcal{H}$. Here, $n^{\prime}=\operatorname{dim} \mathcal{K}_{a}^{\min }$. Set

$$
\mathbf{i}_{h}\langle w\rangle=\sum_{i=1}^{n^{\prime}}\left\langle e_{i}, w\right\rangle_{\mathcal{H}} e_{i} .
$$

In fact, $\left\langle e_{i}, \cdot\right\rangle_{\mathcal{H}} \in \mathcal{W}^{*}$, as we see later. Then, the Malliavin covariance matrix of $\left(R_{1}^{\varepsilon}(w ; h) / \varepsilon, \mathbf{i}_{h}\right) \in \mathbb{D}_{\infty}\left(\mathcal{V} \times T_{h} \mathcal{K}_{a}^{\min }\right)$ is given by

$$
\sigma\left[\left(R_{1}^{\varepsilon}(\cdot ; h) / \varepsilon, \mathbf{i}_{h}\right)\right]=\left(\begin{array}{cc}
\sigma\left[R_{1}^{\varepsilon}(\cdot ; h) / \varepsilon\right] & \Xi \\
\Xi^{*} & \operatorname{Id}_{n^{\prime}}
\end{array}\right)
$$

where $\Xi \in \mathcal{V} \otimes\left(T_{h} \mathcal{K}_{a}^{\min }\right)^{*}$ which is defined by $e \mapsto \Pi_{\mathcal{V}} \int_{0}^{1} J_{1}^{\varepsilon, h} K_{t}^{\varepsilon, h} \mathbf{V}\left(X_{t}^{\varepsilon, h}\right) e^{\prime}(t) d t$

The following lemma is essentially [47, Lemma 6.3], rewritten in a rough path way. Let

$$
\lambda:=\inf _{h \in \mathcal{K}_{a}^{\min }} \inf _{z \in \mathcal{V}:|z|=1} z^{*} \sigma\left[\psi_{1}\right](h) z>0
$$

be the infimum over $h$ of the smallest eigenvalue of $\sigma\left[\psi_{1}\right](h)$.

LEMmA 3.7. There exist $\gamma_{0}, \varepsilon_{0} \in(0,1]$ such that the smallest eigenvalue of $\sigma\left[\left(R_{1}^{\varepsilon}(\cdot ; h) / \varepsilon, \mathbf{i}_{h}\right)\right](w)$ is greater than $(\lambda \wedge 1) / 2$ if $\left\|(\varepsilon \mathbf{W})^{1}\right\|_{\alpha, 4 m}^{4 m}+\left\|(\varepsilon \mathbf{W})^{2}\right\|_{2 \alpha, 2 m}^{2 m}<$ $\gamma_{0}^{4 m}$ (that is $\varepsilon \mathbf{W} \in U_{\gamma_{0}}$ ), $h \in \mathcal{K}_{a}^{\min }$ and $0 \leqslant \varepsilon \leqslant \varepsilon_{0}$. Moreover, both $\gamma_{0}$ and $\varepsilon_{0}$ can be chosen independent of $h \in \mathcal{K}_{a}^{\min }$.

Proof. The covariance matrix $\sigma\left[\left(R_{1}^{\varepsilon}(\cdot ; h) / \varepsilon, \mathbf{i}_{h}\right)\right](w)$ is of the form $A\left(\tau_{h}(\varepsilon \mathbf{W})\right.$, $\left.\lambda^{\varepsilon}\right)$, where

(i) $A: G \Omega_{\alpha, 4 m}^{B}\left(\mathbb{R}^{r+1}\right) \rightarrow L\left(\mathcal{V} \times T_{h} \mathcal{K}_{a}^{\min }, \mathcal{V} \times T_{h} \mathcal{K}_{a}^{\min }\right) \cong \operatorname{Mat}\left(n+n^{\prime}, n+n^{\prime}\right)$ is a certain continuous map,

(ii) $\tau_{h}$ stands for the Young translation by $h$, 
(iii) $\left(\tau_{h}(\varepsilon \mathbf{W}), \lambda^{\varepsilon}\right)$ stands for the Young pairing of $\tau_{h}(\varepsilon \mathbf{W})$ and $\lambda^{\varepsilon}$. At $(\mathbf{h}, 0)$ (or equivalently when $\varepsilon=0$ ) the covariance matrix becomes

$$
A(\mathbf{h}, 0)=\left(\begin{array}{cc}
\sigma\left[\psi_{1}\right](h) & O \\
O & \operatorname{Id}_{n^{\prime}}
\end{array}\right)
$$

which is clearly greater than or equal to $\lambda \wedge 1$ as a quadratic form. (Since $T_{h} \mathcal{K}_{a}^{\text {min }} \subset$ ker $D \psi_{1}(h)$, the 'off-diagonal' components are zero.) Hence we can find $\gamma_{0}$ and $\varepsilon_{0}$ as in the statement for each $h$.

Since $A$ and the Young translation/pairing are Lipschitz continuous on any bounded set, we may take $\gamma_{0}$ and $\varepsilon_{0}$ independently from $h \in \mathcal{K}_{a}^{\min }$.

At the end of this subsection we discuss a large deviation principle of FreidlinWentzell type for conditional measures. Using the upper estimate of the large deviation principle, we prove that contributions from a subset away from $\mathcal{L}\left(\mathcal{K}_{a}^{\mathrm{min}}\right)$ is negligibly small in the proof of the asymptotic expansions. Note that we do not need the lower estimate, which probably does not hold true under our assumptions.

Let $\theta_{a}^{\varepsilon}$ be a finite Borel measure on $\mathcal{W}$ which corresponds to the positive Watanabe distribution $\delta_{a}\left(Y_{1}^{\varepsilon}\right)$ via Sugita's theorem [46]. Since $\mathcal{L}$ is quasisurely defined, we can lift this measure to a measure $\mu_{a}^{\varepsilon}:=(\varepsilon \mathcal{L})_{*}\left[\theta_{x, a}^{\varepsilon}\right]$ on $G \Omega_{\alpha, 4 m}^{B}\left(\mathbb{R}^{d}\right)$. In other words, $\mu_{a}^{\varepsilon}$ is the law of the random variable $\varepsilon \mathbf{W}=\mathcal{L}(\varepsilon w)$ under $\theta_{a}^{\varepsilon}$.

Set a rate function $I: G \Omega_{\alpha, 4 m}^{B}\left(\mathbb{R}^{n}\right) \rightarrow[0, \infty]$ as follows;

$$
I(\mathbf{w})= \begin{cases}\|h\|_{\mathcal{H}}^{2} / 2 & \left(\text { if } \mathbf{w}=\mathcal{L}(h) \text { for some } h \in \mathcal{K}_{a}\right), \\ \infty & \text { (otherwise) }\end{cases}
$$

This rate function $I$ is actually good under (A1). (We can prove this by using Lyons' continuity theorem and the goodness of the rate function for the usual Schilder-type large deviation on the rough path space.)

THEOREM 3.8. Assume (A1) and (A2). Then, the family $\left\{\mu_{a}^{\varepsilon}\right\}_{\varepsilon>0}$ of finite measures satisfies the following upper bound of a large deviation principle on $G \Omega_{\alpha, 4 m}^{B}\left(\mathbb{R}^{d}\right)$ as $\varepsilon \searrow 0$ with a good rate function $I$, that is, for any closed set $A \subset G \Omega_{\alpha, 4 m}^{B}\left(\mathbb{R}^{d}\right)$,

$$
\limsup _{\varepsilon \searrow 0} \varepsilon^{2} \log \mu_{a}^{\varepsilon}(A) \leqslant-\inf _{\mathbf{w} \in A} I(\mathbf{w}) .
$$

Proof. This was proved in Inahama [27] (under slightly stronger assumptions). The keys of the proof are Kusuoka-Stroock's estimate (2.4) and the integration by parts formula (3.1) for Watanabe distributions. Hence, the same proof works 
under (A1) and (A2). (The assumptions in [27] are stronger for the lower bound of the LDP.)

3.4. Basic differential geometry on $\mathcal{K}_{a}^{\text {min }}$. In this subsection we recall some basic results on $\mathcal{K}_{a}^{\min }$ and fix notations. All the ingredients in this subsection can be found in [47, Section 6.2]. We assume (A1), (B1), (B2) in this subsection.

Let $\iota: \mathcal{K}_{a}^{\min } \hookrightarrow \mathcal{H}$ denote the inclusion map. The induced Riemannian metric $g$ on $\mathcal{K}_{a}^{\text {min }}$ is given by $g_{h}\langle u, v\rangle=\left\langle\left(\iota_{*}\right)_{h} u,\left(\iota_{*}\right)_{h} v\right\rangle_{\mathcal{H}}$ for $u, v \in T_{h} \mathcal{K}_{a}^{\text {min }}$ and $h \in \mathcal{K}_{a}^{\text {min }}$. The Riemannian volume measure is denoted by $\omega$.

Let $\mathcal{A}_{h}: T_{h} \mathcal{K}_{a}^{\min } \times T_{h} \mathcal{K}_{a}^{\min } \rightarrow\left(T_{h} \mathcal{K}_{a}^{\min }\right)^{\perp}$ be the second fundamental form defined by

$$
\mathcal{A}_{h}\langle u, v\rangle=\pi^{\perp}\left[\nabla_{\left(\iota_{*}\right)_{h} u}\left(\iota_{*}\right)_{h} v\right] \quad \text { for } u, v \in T_{h} \mathcal{K}_{a}^{\min } \text { and } h \in \mathcal{K}_{a}^{\min } .
$$

Here, $\nabla$ denotes the flat connection on $\mathcal{H}$ and $\pi^{\perp}$ is a short hand for the orthogonal projection from $\mathcal{H}$ onto the orthogonal complement of $\left(\iota_{*}\right)_{h}\left[T_{h} \mathcal{K}_{a}^{\mathrm{min}}\right]$. (Precisely, $v$ should be extended to a vector field near $h$.)

Set $\mathbf{i}_{h} \in \mathbb{D}_{\infty}\left(T_{h} \mathcal{K}_{a}^{\min }\right)$ by

$$
\left\langle u, \mathbf{i}_{h}\langle w\rangle\right\rangle_{T_{h} \mathcal{K}_{a}^{\min }}=\left\langle\left(\iota_{*}\right)_{h} u, w\right\rangle_{\mathcal{H}} \quad \text { for } u \in T_{h} \mathcal{K}_{a}^{\min }, h \in \mathcal{K}_{a}^{\min } .
$$

Precisely, the right hand side above is the stochastic extension of the bounded linear map $\left\langle\left(l_{*}\right)_{h} u, \cdot\right\rangle_{\mathcal{H}}: \mathcal{H} \rightarrow \mathbb{R}$. However, we see in the next section (Proposition 4.3) that it in fact extends to a bounded linear map from $\mathcal{W}$ to $\mathbb{R}$.

Define $\mathbf{a}_{h} \in \mathbb{D}_{\infty}\left(T_{h}^{*}\left(\mathcal{K}_{a}^{\min }\right) \otimes T_{h}^{*}\left(\mathcal{K}_{a}^{\min }\right)\right)$ by

$$
\mathbf{a}_{h}(w)\langle u, v\rangle=\left\langle\mathcal{A}_{h}\langle u, v\rangle, w\right\rangle_{\mathcal{H}} \quad \text { for } u, v \in T_{h} \mathcal{K}_{a}^{\min }, h \in \mathcal{K}_{a}^{\min } .
$$

For the same reason as above, this also extends to a bounded linear map from $\mathcal{W}$ (see a local expression in (3.38) below).

An important remark is that

$$
\mathbf{a}_{h}(h)\langle u, v\rangle=-\langle u, v\rangle_{T_{h} \mathcal{K}_{a}^{\min }} \quad \text { for } u, v \in T_{h} \mathcal{K}_{a}^{\min }, h \in \mathcal{K}_{a}^{\min } .
$$

Note that (3.37) is nontrivial and is a consequence of the fact that $\mathcal{K}_{a}^{\min }$ is a subset of a sphere in $\mathcal{H}$ centered at 0 . It can be checked as follows. First, the problem reduces to the case of two-dimensional sphere embedded in three (or higher) dimensional vector space in a standard way. Next, the case of the two-dimensional sphere can be shown by straightforward computation. Set

We write these quantities in a local coordinate chart $\left(U ; \theta^{1}, \ldots, \theta^{n^{\prime}}\right)$ of $\mathcal{K}_{a}^{\min }$.

$$
\left(\iota_{*}\right)_{h}\left(\frac{\partial}{\partial \theta^{i}}\right)=\left(\frac{\partial \iota}{\partial \theta^{i}}\right)_{h}, \quad g_{i j}(h)=\left\langle\left(\frac{\partial \iota}{\partial \theta^{i}}\right)_{h},\left(\frac{\partial \iota}{\partial \theta^{j}}\right)_{h}\right\rangle_{\mathcal{H}}
$$




$$
G(h)=\left(g_{i j}(h)\right)_{1 \leqslant i, j \leqslant n^{\prime}} \quad \text { and } \quad\left(e_{i}\right)_{h}:=\left\{G(h)^{-1 / 2}\right\}^{i j}\left(\frac{\partial}{\partial \theta^{j}}\right)_{h} .
$$

(Summation over repeated indices are omitted.) By way of construction, $\left\{\left(e_{i}\right)_{h}\right\}_{i=1}^{n^{\prime}}$ is an orthonormal basis of $T_{h} \mathcal{K}_{a}^{\text {min }}$. Using this we can write down $\mathbf{i}_{h}$ and $\mathbf{a}_{h}$ as follows:

$$
\begin{gathered}
\mathbf{i}_{h}(w)=\left\langle\left(\iota_{*}\right)_{h}\left(e_{i}\right)_{h}, w\right\rangle_{\mathcal{H}}\left(e_{i}\right)_{h}, \\
\mathbf{a}_{h}(w)\left\langle\left(e_{i}\right)_{h},\left(e_{j}\right)_{h}\right\rangle=G(h)_{i k}^{-1 / 2} \mathbf{a}_{h}(w)\left\langle\left(\frac{\partial \iota}{\partial \theta^{k}}\right)_{h},\left(\frac{\partial \iota}{\partial \theta^{l}}\right)_{h}\right\rangle_{h} G(h)_{l j}^{-1 / 2} \\
=G(h)_{i k}^{-1 / 2}\left[\left\langle\left(\frac{\partial^{2} \iota}{\partial \theta^{k} \partial \theta^{l}}\right)_{h}, w\right\rangle_{\mathcal{H}}\right. \\
\\
\left.-\left\langle\left(\frac{\partial^{2} \iota}{\partial \theta^{k} \partial \theta^{l}}\right)_{h},\left(\iota_{*}\right)_{h}\left(e_{m}\right)_{h}\right\rangle_{\mathcal{H}}\left\langle\left(\iota_{*}\right)_{h}\left(e_{m}\right)_{h}, w\right\rangle_{\mathcal{H}}\right] G(h)_{l j}^{-1 / 2} .
\end{gathered}
$$

Define

$$
\Delta^{(h)}(w)=\delta_{0}\left(\mathbf{i}_{h}(w)\right) \in \tilde{\mathbb{D}}_{-\infty} \quad \text { and } \quad D^{(h)}(w)=\operatorname{det}\left[-\mathbf{a}_{h}(w)\right] \in \mathbb{D}_{\infty},
$$

where $\delta_{0}$ stands for the delta function at 0 on $T_{h} \mathcal{K}_{a}^{\min }$. Note that Malliavin covariance matrix of $\mathbf{i}_{h}(w)$ is the identity matrix. In a local coordinate chart, $\Delta^{(h)}$ and $D^{(h)}$ can be written as follows:

$$
\Delta^{(h)}(w)=\sqrt{\operatorname{det} G(h)} \cdot \delta_{0}\left(\left\{\left\langle\left(\frac{\partial \iota}{\partial \theta^{i}}\right)_{h}, w\right\rangle_{\mathcal{H}}\right\}_{i=1}^{n^{\prime}}\right),
$$

where $\delta_{0}$ stands for the delta function at 0 on $\mathbb{R}^{n^{\prime}}$, and

$$
\begin{aligned}
D^{(h)}(w)= & \operatorname{det} G(h)^{-1} \\
& \times \operatorname{det}\left[\left\{-\left\langle\left(\frac{\partial^{2} \iota}{\partial \theta^{i} \partial \theta^{j}}\right)_{h}, w\right\rangle_{\mathcal{H}}+\left\langle\left(\frac{\partial^{2} \iota}{\partial \theta^{i} \partial \theta^{j}}\right)_{h},\left(\iota_{*}\right)_{h}\left(e_{m}\right)_{h}\right\rangle_{\mathcal{H}}\right.\right. \\
& \left.\left.\times\left\langle\left(\iota_{*}\right)_{h}\left(e_{m}\right)_{h}, w\right\rangle_{\mathcal{H}}\right\}_{i, j=1}^{n^{\prime}}\right] .
\end{aligned}
$$

Hence,

$$
\begin{aligned}
& D^{(h)}(w) \Delta^{(h)}(w)=\frac{1}{\sqrt{\operatorname{det} G(h)}} \\
& \quad \times \operatorname{det}\left[\left\{-\left\langle\left(\frac{\partial^{2} \iota}{\partial \theta^{i} \partial \theta^{j}}\right)_{h}, w\right\rangle_{\mathcal{H}}\right\}_{i, j=1}^{n^{\prime}}\right] \cdot \delta_{0}\left(\left\{\left\langle\left(\frac{\partial \iota}{\partial \theta^{i}}\right)_{h}, w\right\rangle_{\mathcal{H}}\right\}_{i=1}^{n^{\prime}}\right) \in \tilde{\mathbb{D}}_{-\infty} .
\end{aligned}
$$


Since $\mathcal{K}_{a}^{\min } \ni h \mapsto D^{(h)} \Delta^{(h)} \in \tilde{\mathbb{D}}_{-\infty}$ is continuous, the $\tilde{\mathbb{D}}_{-\infty}$-valued integration

$$
\int_{U} D^{(h)} \Delta^{(h)} \omega(d h)
$$

is well defined, where $\omega(d h)=\sqrt{\operatorname{det} G(h)} d \theta^{1} \cdots d \theta^{n^{\prime}}$ is the Riemannian volume measure.

\section{Some computations of skeleton ODE}

In this section we compute the skeleton ODE under (A1) and (B1). We do not assume (A2), (B2) or (B3) for a while. The main point is that if $h \in \mathcal{K}_{a}^{\min }$ then $\phi(h)$ satisfies (the configuration component of) a Hamiltonian ODE for a naturally defined Hamiltonian. The key in the proof of this fact is the Lagrange multiplier method. This kind of argument has been known for a long time. (See probabilistic literatures such as Bismut [8], Ben Arous [7] or sub-Riemannian geometric literatures such as $[11,40,42]$.) Our exposition basically follows Rifford [42, Section 2.2]. However, we take a new look at this well-known argument from a viewpoint of rough path theory and slightly modify it. For example, the Lagrange multiplier $q(h)$, the key quantity in this argument, can be understood in a rough path way.

First we give some formulas for the skeleton ODE (2.2). In the sequel $D$ also stands for the Fréchet derivative on $\mathcal{H}$. Let $\phi_{t}(h)$ be the solution of the skeleton ODE driven by $h \in \mathcal{H}$ and set $\psi_{t}(h)=\Pi_{\mathcal{V}} \phi_{t}(h)$. For simplicity, we set $\mathbf{V}=$ $\left[V_{1}, \ldots, V_{r}\right]$ which is a $(d \times r)$-matrix.

By straightforward computation,

$$
D \phi_{t}(h)\langle k\rangle=J_{t}(h) \int_{0}^{t} J_{s}(h)^{-1} \mathbf{V}\left(\phi_{s}(h)\right) d k_{s} \quad(k \in \mathcal{H}) .
$$

From this expression, $D \phi_{t}(h)$ and $D \psi_{t}(h)=\Pi_{\mathcal{V}} D \phi_{t}(h)$ extend to continuous linear maps from $\mathcal{W}$ to $\mathbb{R}^{d}$ and to $\mathcal{V}$, respectively. In a similar way, we have

$$
\begin{aligned}
D^{2} \phi_{t}(h)\langle k, \hat{k}\rangle= & J_{t}(h) \int_{0}^{t} J_{s}(h)^{-1}\left\{\nabla^{2} \mathbf{V}\left(\phi_{s}(h)\right)\left\langle D \phi_{s}(h)\langle k\rangle, D \phi_{s}(h)\langle\hat{k}\rangle, d h_{s}\right\rangle\right. \\
& +\nabla \mathbf{V}\left(\phi_{s}(h)\right)\left\langle D \phi_{s}(h)\langle k\rangle, d \hat{k}_{s}\right\rangle \\
& \left.+\nabla \mathbf{V}\left(\phi_{s}(h)\right)\left\langle D \phi_{s}(h)\langle\hat{k}\rangle, d k_{s}\right\rangle\right\} \quad\left(k, k^{\prime} \in \mathcal{H}\right)
\end{aligned}
$$

and $D^{2} \psi_{t}(h)=\Pi_{\mathcal{V}} D^{2} \phi_{t}(h)$. Note that $\left|D^{2} \phi_{t}(h)\langle k, \hat{k}\rangle\right| \leqslant C\|k\|_{\mathcal{H}}\|\hat{k}\|_{\mathcal{W}}$ for some positive constant $C=C(h)$ which actually depends only on $\|h\|_{\mathcal{H}}$. 
We apply the Lagrange multiplier method to $\|\cdot\|_{\mathcal{H}}^{2} / 2$ under the constraint $\psi_{1}=a$. Due to non-degeneracy of $D \psi_{1}(h)$, we have the following; for any $h \in \mathcal{K}_{a}^{\min }$, there exists a unique $q=q(h) \in \mathcal{V}^{*} \cong \mathcal{V}$ such that the function

$$
\mathcal{H} \ni \hat{h} \mapsto \frac{1}{2}\|\hat{h}\|_{\mathcal{H}}^{2}-\left\langle q(h), \psi_{1}(\hat{h})-a\right\rangle_{\mathcal{V}}
$$

is stationary at $h$, that is,

$$
\langle h, k\rangle_{\mathcal{H}}=\left\langle q(h), D \psi_{1}(h)\langle k\rangle\right\rangle_{\mathcal{V}} \quad\left(k \in \mathcal{H}, h \in \mathcal{K}_{a}^{\min }\right) .
$$

Hence, $\langle h, \cdot\rangle_{\mathcal{H}} \in \mathcal{W}^{*}$ for any $h \in \mathcal{K}_{a}^{\min }$.

If we choose an orthonormal basis of $\mathcal{V}$ and set $n=\operatorname{dim} \mathcal{V}$, then we can easily see from the above relation and (A1) that $q(h)$ has the following explicit expression in this coordinate system:

$$
q(h)^{i}=\sum_{j=1}^{n}\left\{\sigma\left[\psi_{1}\right](h)^{-1}\right\}_{i j} D \psi_{1}^{j}(h)\langle h\rangle \quad(1 \leqslant i \leqslant n) .
$$

Therefore, if we assume (B2) in addition, then $h \rightarrow q(h)$ is smooth from $\mathcal{K}_{a}^{\min }$ to $\mathcal{V}$. (We see later that $h \rightarrow q(h)$ extends to a continuous map from the geometric rough path space under (A1) and (B1).)

We now introduce a Hamiltonian and a Hamiltonian ODE which are naturally associated with the skeleton ODE. Define

$$
H(x, p)=\frac{1}{2} \sum_{i=1}^{r}\left\langle p, V_{i}(x)\right\rangle^{2} \quad\left((x, p) \in \mathbb{R}^{d} \times \mathbb{R}^{d} \cong T^{*} \mathbb{R}^{d}\right),
$$

where the bracket denotes the pairing of a covector and a tangent vector. This is called a Hamiltonian function and clearly smooth on $\mathbb{R}^{d} \times \mathbb{R}^{d} \cong T^{*} \mathbb{R}^{d}$.

The Hamiltonian ODE is given by

$$
\begin{gathered}
\frac{d x_{t}}{d t}=\frac{\partial H}{\partial p}\left(x_{t}, p_{t}\right)=\sum_{i=1}^{r} V_{i}\left(x_{t}\right)\left\langle p_{t}, V_{i}\left(x_{t}\right)\right\rangle, \\
\frac{d p_{t}}{d t}=-\frac{\partial H}{\partial x}\left(x_{t}, p_{t}\right)=-\sum_{i=1}^{r}\left\langle p_{t}, \nabla V_{i}\left(x_{t}\right)\right\rangle\left\langle p_{t}, V_{i}\left(x_{t}\right)\right\rangle .
\end{gathered}
$$

Since we always assume $x_{0}=x$, dependence on $x$ will be suppressed. The solution with the initial condition $\left(x, p_{0}\right)$ is denoted by $\left(x_{t}\left(p_{0}\right), p_{t}\left(p_{0}\right)\right)$. 
In the natural coordinate $x=\left(x^{1}, \ldots, x^{d}\right)$ of $\mathbb{R}^{d}$, we can write $p=\sum_{j=1}^{d} p_{j} d x^{j}$ and $V_{i}(x)=\sum_{j=1}^{d} V_{i}^{j}(x)\left(\partial / \partial x^{j}\right)$. Moreover,

$$
\left\langle p, V_{i}(x)\right\rangle=\sum_{j=1}^{d} p_{j} V_{i}^{j}(x) \in \mathbb{R} \quad \text { and } \quad\left\langle p, \nabla V_{i}(x)\right\rangle=\sum_{j, k=1}^{d} p_{j} \frac{\partial V_{i}^{j}}{\partial x^{k}}(x) d x^{k} .
$$

Therefore, the Hamiltonian ODE (4.5)-(4.6) above can also be expressed as follows:

$$
\begin{gathered}
\frac{d x_{t}^{k}}{d t}=\frac{\partial H}{\partial p_{k}}\left(x_{t}, p_{t}\right)=\sum_{i=1}^{r} V_{i}^{k}\left(x_{t}\right) \sum_{j=1}^{d} p_{j, t} V_{i}^{j}\left(x_{t}\right) \\
\frac{d p_{k, t}}{d t}=-\frac{\partial H}{\partial x^{k}}\left(x_{t}, p_{t}\right)=-\sum_{i=1}^{r} \sum_{j=1}^{d} p_{j, t} \frac{\partial V_{i}^{j}}{\partial x^{k}}\left(x_{t}\right) \sum_{l=1}^{d} p_{l, t} V_{i}^{l}\left(x_{t}\right) .
\end{gathered}
$$

We do not prove that a unique global solution exists for a given initial condition because it is unnecessary for our purpose.

A remarkable fact is that the solution $\phi_{t}(h)$ of the skeleton ODE for $h \in \mathcal{K}_{a}^{\text {min }}$ can be expressed as the 'configuration component' of the Hamiltonian ODE.

Proposition 4.1. Assume (A1) and (B1). Let $h \in \mathcal{K}_{a}^{\min }$ and denote by $q(h)$ the Lagrange multiplier given in (4.3). Then, a unique global solution of the Hamiltonian ODE with the initial condition $p_{0}=\left\langle q(h), \Pi_{\mathcal{V}} J_{1}(h) \bullet\right\rangle_{\mathcal{V}} \in$ $T_{x}^{*} \mathbb{R}^{d}\left(\cong \mathbb{R}^{d}\right)$ exists and satisfies that $x_{t}\left(p_{0}\right) \equiv \phi_{t}(h)$. Moreover, $h_{t}^{i} \equiv \int_{0}^{t}\left\langle p_{s}\left(p_{0}\right)\right.$, $\left.V_{i}\left(x_{s}\left(p_{0}\right)\right)\right\rangle d s(1 \leqslant i \leqslant r)$.

Proof. For simplicity of notations we assume that $\mathcal{V}=\mathbb{R}^{n} \times\left\{\mathbf{0}_{d-n}\right\}$, where $n=$ $\operatorname{dim} \mathcal{V}$. (Without loss of generality we may do so.) Then, $\Pi_{\mathcal{V}}=\left[\operatorname{Id}_{n} \mid \mathbf{0}_{n \times(d-n)}\right]$. $J$ and $J^{-1}$ are $d \times d$ matrices and $\mathbf{V}$ is a $d \times r$ matrix. $q(h)$ and $p$ are regarded as an $n$-dimensional and a $d$-dimensional row vector, respectively. Since $H$ is smooth, the uniqueness is obvious if a solution exists.

From (4.1) and (4.3) we have

$$
\int_{0}^{1}\left\langle h_{s}^{\prime}, k_{s}^{\prime}\right\rangle d s=\int_{0}^{1} q(h) \Pi_{\mathcal{V}} J_{1}(h) J_{s}^{-1}(h) \mathbf{V}\left(\phi_{s}(h)\right) k_{s}^{\prime} d s .
$$

Since $k \in \mathcal{H}$ is arbitrary, we can easily see that

$$
\left(h_{t}^{1}, \ldots, h_{t}^{r}\right) \equiv \int_{0}^{t} q(h) \Pi_{\mathcal{V}} J_{1}(h) J_{s}^{-1}(h) \mathbf{V}\left(\phi_{s}(h)\right) d s .
$$


Define $x_{t}=\phi_{t}(h)$ and $p_{t}=q(h) \Pi_{\mathcal{V}} J_{1}(h) J_{t}^{-1}(h)$. Clearly, $p_{0}=q(h) \Pi_{\mathcal{V}} J_{1}(h)$ and $\left(h_{t}^{1}, \ldots, h_{t}^{r}\right) \equiv \int_{0}^{t} p_{s} \mathbf{V}\left(\phi_{s}(h)\right) d s$. The skeleton ODE can be rewritten as

$$
d \phi_{t}(h)=\sum_{i=1}^{r} V_{i}\left(\phi_{t}(h)\right) d h_{t}^{i}=\sum_{i=1}^{r} V_{i}\left(\phi_{t}(h)\right)\left[p_{t} V_{i}\left(\phi_{t}(h)\right)\right] d t,
$$

which means that (4.5) is satisfied. From the ODE for $J_{t}^{-1}=K_{t}$, we can see that

$$
\begin{aligned}
p_{t}^{\prime} & =q(h) \Pi_{\mathcal{V}} J_{1}(h)\left[-J_{t}^{-1}(h) \sum_{i=1}^{r} \nabla V_{i}\left(\phi_{t}(h)\right)\left(h_{t}^{i}\right)^{\prime}\right] \\
& =-p_{t} \sum_{i=1}^{r} \nabla V_{i}\left(\phi_{t}(h)\right)\left[p_{t} V_{i}\left(\phi_{t}(h)\right)\right] .
\end{aligned}
$$

Thus, (4.6) is also satisfied.

REMARK 4.2. In Proposition 4.1 above, the Hamiltonian $H$ is of course constant along the trajectory of $t \mapsto\left(x_{t}\left(p_{0}\right), p_{t}\left(p_{0}\right)\right)$. From the explicit form of $h$ we can easily see that the constant is $d_{a}^{2} / 2=\|h\|_{\mathcal{H}}^{2} / 2>0$. Furthermore, this implies that the smooth path $t \mapsto x_{t}\left(p_{0}\right)=\phi_{t}(h)$ is regular, that is, its velocity vector never vanishes. Indeed,

$$
\begin{aligned}
\left\langle p_{t}\left(p_{0}\right), \phi_{t}^{\prime}(h)\right\rangle & =\left\langle p_{t}\left(p_{0}\right), \sum_{i=1}^{r} V_{i}\left(\phi_{t}(h)\right)\left\langle p_{t}\left(p_{0}\right), V_{i}\left(\phi_{t}(h)\right)\right\rangle\right\rangle \\
& =2 H\left(x_{t}\left(p_{0}\right), p_{t}\left(p_{0}\right)\right) \equiv d_{a}^{2}>0
\end{aligned}
$$

for any $t$.

Thanks to the above proposition, we know that if $h \in \mathcal{K}_{a}^{\min }$, then both $h$ and $\phi(h)$ are smooth in $t$. This fact is highly nontrivial since a generic element of $\mathcal{H}$ is not even of $C^{1}$. Moreover, we have the following proposition:

Proposition 4.3. Assume (A1), (B1) and (B2). Then, the mappings $(t, h) \mapsto$ $\phi_{t}(h)$ and $(t, h) \mapsto h_{t}$ are smooth from $[0,1] \times \mathcal{K}_{a}^{\min }$ to $\mathbb{R}^{d}$ and to $\mathbb{R}^{r}$, respectively.

Proof. Assume that Hamiltonian ODE (4.5)-(4.6) has a unique global solution for an initial condition $\left(x, \hat{p}_{0}\right)$. Then, a standard cut-off technique and smoothness of $H$ shows that there is an open neighborhood $U$ of $\hat{p}_{0}$ such that (i) a unique global solution exists for any $\left(x, p_{0}\right)$ with $p_{0} \in U$ and (ii) the mapping $[0,1] \times U \ni$ $\left(t, p_{0}\right) \mapsto\left(x_{t}\left(p_{0}\right), p_{t}\left(p_{0}\right)\right) \in \mathbb{R}^{d} \times \mathbb{R}^{d}$ is smooth. 
Since $\mathcal{K}_{a}^{\min } \ni h \mapsto p_{0}=\left\langle q(h), \Pi_{\mathcal{V}} J_{1}(h) \bullet\right\rangle_{\mathcal{V}}$ is smooth under (B2), we see that $(t, h) \mapsto x_{t}\left(p_{0}\right)=\phi_{t}(h)$ is also smooth. Smoothness of $(t, h) \mapsto h_{t}$ is immediate if we recall

$$
\frac{d}{d t}\left(h_{t}^{1}, \ldots, h_{t}^{r}\right) \equiv q(h) \Pi_{\mathcal{V}} J_{1}(h) J_{t}^{-1}(h) \mathbf{V}\left(\phi_{t}(h)\right) .
$$

This completes the proof.

The continuity of the natural lift map $\left.\mathcal{L}\right|_{\mathcal{H}}: \mathcal{H} \hookrightarrow G \Omega_{\alpha, 4 m}^{B}\left(\mathbb{R}^{r}\right)$ means that the rough path topology, restricted on $\mathcal{L}(\mathcal{H})$, is weaker than $\mathcal{H}$-topology. The next proposition claims that on $\mathcal{L}\left(\mathcal{K}_{a}^{\min }\right)$ the two topologies are in fact the same.

Proposition 4.4. Assume (A1) and (B1). Let $h_{1}, h_{2}, \ldots, h_{\infty} \in \mathcal{K}_{a}^{\min }$. Then, $\lim _{j \rightarrow \infty} h_{j}=h_{\infty}$ in $\mathcal{H}$ if and only if $\lim _{j \rightarrow \infty} \mathcal{L}\left(h_{j}\right)=\mathcal{L}\left(h_{\infty}\right)$ in $G \Omega_{\alpha, 4 m}^{B}\left(\mathbb{R}^{r}\right)$.

Proof. By setting $(\mathbf{w}, \lambda)=(\mathcal{L}(h), 0)$ in (3.20), we see that $h \mapsto(h, \phi(h)$, $\left.J(h), J^{-1}(h)\right)$ extends to a continuous map from $G \Omega_{\alpha, 4 m}^{B}\left(\mathbb{R}^{r}\right)$ to $G \Omega_{\alpha^{\prime}}^{H}\left(\mathbb{R}^{r} \oplus \mathbb{R}^{d}\right.$ $\oplus \operatorname{Mat}(d, d) \oplus \operatorname{Mat}(d, d))$ for any $\alpha^{\prime} \in(1 / 3, \alpha-1 /(4 m))$. From this and (4.1) we see that

$$
h \mapsto D \psi_{1}(h)\langle h\rangle=\Pi_{\mathcal{V}} J_{1}(h) \int_{0}^{1} J_{s}(h)^{-1} \mathbf{V}\left(\phi_{s}(h)\right) d h_{s}
$$

extends to a continuous map from $G \Omega_{\alpha, 4 m}^{B}\left(\mathbb{R}^{r}\right)$ since the right hand side can be regarded as a rough path integral along the natural lift of $\left(h, \phi(h), J(h), J^{-1}(h)\right)$. Also, the deterministic Malliavin covariance matrix $h \mapsto \sigma\left[\psi_{1}\right](h)$ extends to a continuous map from $G \Omega_{\alpha, 4 m}^{B}\left(\mathbb{R}^{r}\right)$. Hence, from the explicit expression (4.4), we see that $\mathcal{K}_{a}^{\min } \ni h \mapsto q(h)$ is continuous with respect to the rough path topology. Therefore, as $h \in \mathcal{K}_{a}^{\text {min }}$ varies continuously with respect to the rough path topology,

$$
\frac{d}{d t}\left(h_{t}^{1}, \ldots, h_{t}^{r}\right) \equiv q(h) \Pi_{\mathcal{V}} J_{1}(h) J_{t}^{-1}(h) \mathbf{V}\left(\phi_{t}(h)\right)
$$

varies continuously with respect to $L^{2}\left([0,1], \mathbb{R}^{r}\right)$-topology. Thus $\lim _{j \rightarrow \infty} \mathcal{L}\left(h_{j}\right)=$ $\mathcal{L}\left(h_{\infty}\right)$ in $G \Omega_{\alpha, 4 m}^{B}\left(\mathbb{R}^{r}\right)$ implies $\lim _{j \rightarrow \infty}\left\|h_{j}-h_{\infty}\right\|_{\mathcal{H}}=0$. The converse implication is obvious.

REMARK 4.5. Since the rate function in a Schilder-type large deviation principle on the rough path space is good, $\mathcal{K}_{a}^{\min }$ is compact with respect to $G \Omega_{\alpha, 4 m}^{B}\left(\mathbb{R}^{r}\right)$ topology under (A1). If we assume (B1) in addition, the above proposition implies that it is also compact with respect to $\mathcal{H}$-topology. From this viewpoint, the compactness assumption in (B2) is not strong. 
The following lemma is essentially a rough path version of [47, Lemma 6.2]. We use $(\alpha, 4 m)$-Besov rough path topology instead of the norm $\left\{\left|w_{1}\right|^{2}+\right.$ $\left.\int_{0}^{1}\left|w_{s}\right|^{2} d s\right\}^{1 / 2}$. In the statement of this lemma, a 'neighborhood' means a neighborhood with respect to $\mathcal{H}$-topology. Due to Proposition 4.4, the assertion (i) clearly holds if $\gamma$ is small enough since $\left\{U_{h, \gamma} \mid \gamma>0\right\}$ forms a fundamental system of neighborhood of $\mathcal{L}(h)$ with respect to $(\alpha, 4 m)$-Besov rough path topology.

LEMMA 4.6. Assume (A1), (B1) and (B2). For any $h \in \mathcal{K}_{a}^{\min }$, there exists a coordinate neighborhood $O \subset \mathcal{K}_{a}^{\min }$ of $h$ which satisfies the following property: for any subneighborhood $O^{\prime}$ of $O$, there exists $\gamma>0$ such that:

(i) $\left\{k \in \mathcal{K}_{a}^{\min } \mid \mathcal{L}(k) \in U_{h, \gamma}\right\} \subset O^{\prime}$;

(ii) $\int_{O^{\prime}} D^{(k)} \Delta^{(k)} \omega(d k)=1$ on $\left\{w \in \mathcal{W} \mid \mathcal{L}(w) \in U_{h, \gamma}\right\}$, that is, for any $F \in \tilde{\mathbb{D}}_{\infty}$ such that $F \cdot \mathbf{1}_{\left\{\mathcal{L}(w) \in U_{h, \gamma}\right\}}=F$ a.s., it holds that $\mathbb{E}\left[F \int_{O^{\prime}} D^{(k)} \Delta^{(k)} \omega(d k)\right]=$ $\mathbb{E}[F]$.

Proof. The proof here is essentially the same as the proof of [47, Lemma 6.2]. We use Proposition 4.3 and the inverse function theorem. We denote by $\mathcal{W}_{\alpha, 4 m}^{B}$ the Banach space of all the elements in $\mathcal{W}$ with finite $(\alpha, 4 m)$-Besov norm. The injection $\mathcal{K}_{a}^{\min } \hookrightarrow \mathcal{H}$ is denoted by $\iota$, which will be sometimes omitted when the notation gets too heavy.

Take $h \in \mathcal{K}_{a}^{\min }$ and a coordinate neighborhood $O$ of $h$ arbitrarily. Let $Q$ be an open subset of $\mathbb{R}^{n^{\prime}}$ such that $Q \ni\left(\theta^{1}, \ldots, \theta^{n^{\prime}}\right) \mapsto k(\theta)=k\left(\theta^{1}, \ldots, \theta^{n^{\prime}}\right) \in O$ is diffeomorphic (namely, $k: Q \rightarrow O$ is a chart map).

We consider

$$
\left.\frac{\partial \iota}{\partial \theta^{i}}\right|_{k(\theta)}=\left\{\frac{\partial}{\partial \theta^{i}} k(\theta)_{t}\right\}_{0 \leqslant t \leqslant 1} \text { and }\left.\frac{\partial^{2} \iota}{\partial \theta^{i} \partial \theta^{j}}\right|_{k(\theta)}=\left\{\frac{\partial^{2}}{\partial \theta^{i} \partial \theta^{j}} k(\theta)_{t}\right\}_{0 \leqslant t \leqslant 1} .
$$

An integration by parts on $[0,1]$ immediately yields

$$
\begin{gathered}
\left\langle\left.\frac{\partial \iota}{\partial \theta^{i}}\right|_{k(\theta)}, w\right\rangle_{\mathcal{H}}=\left\langle w_{1}, \frac{\partial}{\partial \theta^{i}} k(\theta)_{1}^{\prime}\right\rangle_{\mathbb{R}^{r}}-\int_{0}^{1}\left\langle w_{t}, \frac{\partial}{\partial \theta^{i}} k(\theta)_{t}^{\prime \prime}\right\rangle_{\mathbb{R}^{r}} d t, \\
\left\langle\left.\frac{\partial^{2} \iota}{\partial \theta^{i} \partial \theta^{j}}\right|_{k(\theta)}, w\right\rangle_{\mathcal{H}}=\left\langle w_{1}, \frac{\partial^{2}}{\partial \theta^{i} \partial \theta^{j}} k(\theta)_{1}^{\prime}\right\rangle_{\mathbb{R}^{r}}-\int_{0}^{1}\left\langle w_{t}, \frac{\partial^{2}}{\partial \theta^{i} \partial \theta^{j}} k(\theta)_{t}^{\prime \prime}\right\rangle_{\mathbb{R}^{r}} d t .
\end{gathered}
$$

From Proposition 4.3, these two functions on the left hand sides above are defined for all $(\theta, w) \in Q \times \mathcal{W}_{\alpha, 4 m}^{B}$ and continuous with respect to the product topology. (In particular, for each fixed $\theta$ these two linear functionals on $\mathcal{H}$ continuously extends to ones on $\mathcal{W}_{\alpha, 4 m}^{B}$ or $\mathcal{W}$.) Moreover, they are smooth in $\theta$ for any fixed $w$ and their derivatives are all continuous in $(\theta, w)$, too. 
Set

$$
\begin{gathered}
f(\theta, w)=-\left[\left\langle\left.\frac{\partial \iota}{\partial \theta^{i}}\right|_{k(\theta)}, w\right\rangle_{\mathcal{H}}\right]_{1 \leqslant i \leqslant n^{\prime}}, \\
J_{f}(\theta, w)=-\left[\left\langle\left.\frac{\partial^{2} \iota}{\partial \theta^{i} \partial \theta^{j}}\right|_{k(\theta)}, w\right\rangle_{\mathcal{H}}\right]_{1 \leqslant i, j \leqslant n^{\prime}},
\end{gathered}
$$

where the latter is the Jacobian matrix of the former with respect to the $\theta$-variable. Then, we can easily check that $f(\theta, k(\theta))=0$ and $J_{f}(\theta, k(\theta))_{i j}=g_{i j}(k(\theta))$, where $\left(g_{i j}\right)$ is the Riemannian metric tensor on $\mathcal{K}_{a}^{\min }$. (We omit the proof because it is a routine. See [47, pages 215-216]. However, we note that the fact that $\mathcal{K}_{a}^{\text {min }}$ is a subset of a sphere is crucial here.) For each fixed $\theta, f$ is an $\mathbb{R}^{n^{\prime}}$-valued linear functional which is non-degenerate in the sense of Malliavin since its Malliavin covariance matrix is given by $\left(g_{i j}\right)$. Also, $J_{f}$ is linear in $w$ and det $J_{f}$ is just a polynomial in $w$.

For each fixed $w$, we use the inverse function theorem for $f(\cdot, w)$ and carefully keep track of the dependence on $w$. We write $h=k\left(\theta_{0}\right), Q_{\rho}\left(\theta_{0}\right)=\left\{\theta|| \theta-\theta_{0} \mid<\right.$ $\rho\} \subset Q$ and $B_{\gamma}(h)=\left\{w \mid\|w-h\|_{\alpha, 4 m-B}<\gamma\right\}$ for $\rho, \gamma>0$.

Then, we can show the existence of $\rho^{\prime}>0$ and $\gamma^{\prime}>0$ such that:

(a) for each $w \in B_{\gamma^{\prime}}(h), Q_{\rho^{\prime}}\left(\theta_{0}\right) \ni \theta \mapsto f(\theta, w) \in f\left(Q_{\rho^{\prime}}\left(\theta_{0}\right), w\right)$ is diffeomorphic and

(b) for each $\rho \in\left(0, \rho^{\prime}\right)$, there exists $\gamma \in\left(0, \gamma^{\prime}\right)$ such that $\cap\left\{f\left(Q_{\rho}\left(\theta_{0}\right), w\right) \mid w \in\right.$ $\left.B_{\gamma}(h)\right\}$ contains $0 \in \mathbb{R}^{n^{\prime}}$ as an interior point and the assertion (i) holds.

(Though it is not difficult, it is not so obvious, either. Find a nice textbook on calculus and modify a proof in it. Note also that the implicit function theorem is not used here.) We write $O_{\rho}=\left\{k(\theta) \mid \theta \in Q_{\rho}\left(\theta_{0}\right)\right\}$, which is the subneighborhood $O^{\prime}$ in the statement of the lemma.

Let $\chi: \mathbb{R}^{n^{\prime}} \rightarrow[0, \infty)$ be a smooth, radial function with compact support such that $\int_{\mathbb{R}^{n^{\prime}}} \chi=1$. It is well known that $\chi_{\kappa}:=\kappa^{-n^{\prime}} \chi(\cdot / \kappa) \rightarrow \delta_{0}$ as $\kappa \searrow 0$ in $\mathcal{S}^{\prime}\left(\mathbb{R}^{n^{\prime}}\right)$. If $\kappa>0$ is sufficiently small,

$$
\int_{Q_{\rho}\left(\theta_{0}\right)} \operatorname{det} J_{f}(\theta, w) \chi_{\kappa}(f(\theta, w)) d \theta=1 \quad \text { for all } w \in B_{\gamma}(h) .
$$

If $F$ is supported in $\left\{w \mid \mathcal{L}(w) \in U_{h, \gamma}\right\} \subset B_{\gamma}(h)$,

$$
\mathbb{E}\left[F \int_{Q_{\rho}\left(\theta_{0}\right)} \operatorname{det} J_{f}(\theta, \cdot) \chi_{\kappa}(f(\theta, \cdot)) d \theta\right]=\mathbb{E}[F] .
$$

There exists constant $l>0$ independent of $\theta$ such that $\chi_{\kappa}(f(\theta, \cdot)) \rightarrow$ $\delta_{0}(f(\theta, \cdot))=\delta_{0}(-f(\theta, \cdot))$ in $\mathbb{D}_{p^{\prime},-l}$-norm for any $p^{\prime} \in(1, \infty)$ uniformly 
in $\theta$. Since $F \in \mathbb{D}_{p, l}$ for some $p=p(l) \in(1, \infty)$, we have

$$
\mathbb{E}\left[F \int_{Q_{\rho}\left(\theta_{0}\right)} \operatorname{det} J_{f}(\theta, \cdot) \delta_{0}(-f(\theta, \cdot)) d \theta\right]=\mathbb{E}[F] .
$$

Since $\omega(d k)=\sqrt{\operatorname{det} G(k(\theta))} d \theta$, where $G=\left(g_{i j}\right)$ is the Riemannian metric tensor, the left hand side is equal to

$$
\mathbb{E}\left[F \int_{O_{\rho}} D^{(k)} \Delta^{(k)} \omega(d k)\right]
$$

Thus, we have shown (ii).

REMARK 4.7. (1) By carefully examining the proof, we can slightly strengthen the assertion (ii), Lemma 4.6 as follows: for any $\varepsilon \in(0,1]$,

$$
\int_{O^{\prime}} D^{(k)}(\varepsilon w) \Delta^{(k)}(\varepsilon w) \omega(d k)=1 \quad \text { on }\left\{w \mid \mathcal{L}(\varepsilon w) \in U_{h, \gamma}\right\}
$$

for the same $\gamma>0$ as in Lemma 4.6. The proof is essentially the same, but we should note that $f(\theta, \varepsilon w), J_{f}(\theta, \varepsilon w)$, and so forth, are well defined since $f(\theta, \cdot), J_{f}(\theta, \cdot)$, and so forth are continuous maps from $\mathcal{W}$.

(2) It is almost obvious that if (i), (ii) in Lemma 4.6 hold for some $\gamma>0$, then they still hold for any $\hat{\gamma} \in(0, \gamma)$. In other words, we may replace $\gamma$ in Lemma 4.6 by any smaller positive constant.

\section{Exponential integrability lemmas}

In this section we see that the positivity of Hessian assumed in (B3) implies exponential integrability of a corresponding quadratic Wiener functional. In this section we assume (A1) and (B1)-(B3).

Let $\left(-\tau_{0}, \tau_{0}\right) \ni \tau \mapsto c(\tau) \in \mathcal{K}_{a}$ be a smooth curve in $\mathcal{K}_{a}$ such that $c(0)=h \in$ $\mathcal{K}_{a}^{\min }$ and $0 \neq k:=c^{\prime}(0) \in \mathcal{H}_{0}(h)$, where we set $\mathcal{H}_{0}(h)=T_{h} \mathcal{K}_{a} \cap\left(T_{h} \mathcal{K}_{a}^{\text {min }}\right)^{\perp}$ as in (B3). Then, a straightforward calculation shows that

$$
\begin{aligned}
\left.\frac{d^{2}}{d \tau^{2}}\right|_{\tau=0} \frac{\|c(\tau)\|_{\mathcal{H}}^{2}}{2}=\left.\frac{d^{2}}{d \tau^{2}}\right|_{\tau=0}\left(\frac{\|c(\tau)\|_{\mathcal{H}}^{2}}{2}-\left\langle q(h), \psi_{1}(c(\tau))-a\right\rangle\right) \\
=\left\|c^{\prime}(0)\right\|_{\mathcal{H}}^{2}+\left\langle c^{\prime \prime}(0), c(0)\right\rangle_{\mathcal{H}}-\left\langle q(h), D \psi_{1}(c(0))\left\langle c^{\prime \prime}(0)\right\rangle\right\rangle \\
\quad-\left\langle q(h), D^{2} \psi_{1}(c(0))\left\langle c^{\prime}(0), c^{\prime}(0)\right\rangle\right\rangle \\
=\|k\|_{\mathcal{H}}^{2}-\left\langle q(h), D^{2} \psi_{1}(h)\langle k, k\rangle\right\rangle .
\end{aligned}
$$

The cancelation above was due to the Lagrange multiplier method (4.3). 
It is known that the symmetric bounded bilinear form $\left\langle q(h), D^{2} \psi_{1}(h)\langle\bullet, \star\rangle\right\rangle$ is Hilbert-Schmidt and so is $\left\langle q(h), D^{2} \psi_{1}(h)\left\langle\pi^{h} \bullet, \pi^{h} \star\right\rangle\right\rangle$, where $\pi^{h}: \mathcal{H} \rightarrow \mathcal{H}_{0}(h)$ is the orthogonal projection. (An explicit form of $\pi^{h}$ will be given in (5.6) below.) As a result, the spectra of their corresponding symmetric operators are discrete except at 0. Consequently, the condition (B3) implies that

$$
\begin{aligned}
C(h) & :=\sup \left\{\frac{1}{2}\left\langle q(h), D^{2} \psi_{1}(h)\left\langle\pi^{h} v, \pi^{h} v^{\prime}\right\rangle\right\rangle_{\mathcal{V}} \mid v, v^{\prime} \in \mathcal{H},\|v\|_{\mathcal{H}}=\left\|v^{\prime}\right\|_{\mathcal{H}}=1\right\} \\
& <\frac{1}{2} .
\end{aligned}
$$

From the explicit expressions of $D^{2} \psi_{1}(h), q(h)$ and $\pi^{h}$ in (4.2), (4.4) and (5.6), we can easily see that

$$
\mathcal{K}_{a}^{\min } \ni h \mapsto \frac{1}{2}\left\langle q(h), D^{2} \psi_{1}(h)\left\langle\pi^{h} \bullet, \pi^{h} \star\right\rangle\right\rangle
$$

is continuous with respect to the topology of bounded bilinear forms. This implies the continuity of $h \mapsto C(h)$ and

$$
C_{\text {max }}:=\max \left\{C(h) \mid h \in \mathcal{K}_{a}^{\min }\right\}<\frac{1}{2} .
$$

Therefore, if $\Xi_{h} \in \mathcal{C}_{2}$ corresponds to the symmetric Hilbert-Schmidt bilinear form in (5.3), namely the bilinear form is equal to $(1 / 2) D^{2} \Xi_{h}$, then $\mathbb{E}\left[e^{c_{1} \Xi_{h}}\right] \leqslant$ $M<\infty$ for certain constants $c_{1}>1$ and $M>0$ independent of $h \in \mathcal{K}_{a}^{\min }$. (We denote by $\mathcal{C}_{i}$ the $i$ th order homogeneous Wiener chaos.)

The following lemma is [47, Lemma 6.1], which states that the quadratic Wiener functionals that appear as the second term of the Taylor-like expansion of Itô map is exponentially integrable with respect to the conditional Gaussian measure. Though $g_{2}$ can be written as a iterated stochastic integral of second order, we do not use it. Our proof uses rough path theory and can be found in [26] in a more detailed way. For the definition of $\mathbf{i}_{h}$ and $g_{i}(i=0,1,2, \ldots)$ see (3.35) and (3.30), respectively.

Lemma 5.1. Assume (A1) and (B1)-(B3). Then, there exists a constant $c_{1}>1$ which is independent of $h \in \mathcal{K}_{a}^{\min }$ and satisfies that

$$
\sup _{h \in \mathcal{K}_{a}^{\min }} \mathbb{E}\left[\exp \left(c_{1}\left\langle q(h), g_{2}(w ; h)\right\rangle_{\mathcal{V}}\right) \delta_{0}\left(g_{1}(w ; h), \mathbf{i}_{h}\langle w\rangle\right)\right]<\infty .
$$

Here, $\delta_{0}$ is the Dirac delta function on $\mathcal{V} \times T_{h} \mathcal{K}_{a}^{\min } \cong \mathbb{R}^{n+n^{\prime}}$.

Proof. Let $h \in \mathcal{K}_{a}^{\min }$ and let $\left\{e_{i}(h)\right\}_{i=1}^{n^{\prime}}$ be an orthonormal basis of $T_{h} \mathcal{K}_{a}^{\min } \subset \mathcal{H}$. Due to Proposition 4.3, $\left\langle e_{i}(h), \cdot\right\rangle_{\mathcal{H}} \in \mathcal{H}^{*}$ naturally extends to an element in $\mathcal{W}^{*}$. 
Recall that $g_{1}(\cdot ; h) \in \mathcal{W}^{*}$ is the continuous extension of $D \psi_{1}(h) \in \mathcal{H}^{*}$. Then,

$$
\mathcal{H}_{0}(h)=\operatorname{ker} g_{1}(\cdot ; h) \cap\left(\bigcap_{i=1}^{n^{\prime}} \operatorname{ker}\left\langle e_{i}(h), \cdot\right\rangle_{\mathcal{H}}\right)=\operatorname{ker} g_{1}(\cdot ; h) \cap \operatorname{ker} \mathbf{i}_{h},
$$

which may depend on $h$, but not on the choice of the orthonormal basis. It is easy to see that $\mathcal{H}_{0}(h)^{\perp}$ is a finite-dimensional subspace of dimension $n+n^{\prime}$. Denote by $\mathcal{W}_{0}(h)$ the closure of $\mathcal{H}_{0}(h)$ with respect to the topology of $\mathcal{W}$. The orthogonal projection $\pi^{h}: \mathcal{H} \rightarrow \mathcal{H}_{0}(h)$ naturally extends to a continuous projection $\pi^{h}: \mathcal{W} \rightarrow \mathcal{W}_{0}(h)$, which we denote by the same symbol.

The finite Borel measure corresponding to the positive Watanabe distribution $\delta_{0}\left(g_{1}(\cdot ; h), \mathbf{i}_{h}\right)$ via Sugita's theorem $[46]$ is $(2 \pi)^{-\left(n+n^{\prime}\right) / 2}\left\{\operatorname{det} \sigma\left[\psi_{1}\right](h)\right\}^{-1 / 2} \cdot \pi_{*}^{h} \mu$, which is a constant multiple of a Gaussian probability measure $\pi_{*}^{h} \mu$ supported on $\mathcal{W}_{0}(h)$. It is clear that $\left(\mathcal{W}_{0}(h), \mathcal{H}_{0}(h), \pi_{*}^{h} \mu\right)$ is an abstract Wiener space.

Let us now identify $\mathcal{V} \cong \mathbb{R}^{n}$ by choosing an orthonormal basis of $\mathcal{V}$. (The choice is independent of $h$.) Then we have explicitly that

$$
\begin{aligned}
\pi^{h} w & =w-\sum_{l, l^{\prime}=1}^{n}\left\{\sigma\left[\psi_{1}\right](h)^{-1}\right\}_{l l^{\prime}} g_{1}(w ; h)^{l} \cdot{ }^{\sharp} g_{1}(\cdot ; h)^{l^{\prime}}-\sum_{i=1}^{n^{\prime}}\left\langle e_{i}(h), w\right\rangle e_{i}(h) \\
& =w-\sum_{l, l^{\prime}=1}^{n}\left\{\sigma\left[\psi_{1}\right](h)^{-1}\right\}_{l l^{\prime}} g_{1}(w ; h)^{l} \cdot{ }^{\sharp} g_{1}(\cdot ; h)^{l^{\prime}}-\mathbf{i}_{h}\langle w\rangle
\end{aligned}
$$

and we set $\left(\pi^{h}\right)^{\perp} w=w-\pi^{h} w \in \mathcal{H}$. Here, $g_{1}(w ; h)^{l}$ is the $l$ th component of $g_{1}(w ; h)=D \psi_{1}(h)\langle w\rangle,\left\{\sigma\left[\psi_{1}\right](h)^{-1}\right\}_{l l^{\prime}}$ is the $\left(l, l^{\prime}\right)$ th component of the inverse of the deterministic covariance matrix $\sigma\left[\psi_{1}\right](h)$ at $h,{ }^{\sharp} g_{1}(\cdot ; h)^{l}$ is the unique element in $\mathcal{H}$ that corresponds to $g_{1}(\cdot ; h)^{l} \in \mathcal{W}^{*} \subset \mathcal{H}^{*}$ via the Riesz isometry. Note that ${ }^{\sharp} g_{1}(\cdot ; h)^{l}$ and $e_{i}(h)$ are orthogonal for any $l$ and $i$.

By abusing notations we write $\pi^{h} \mathbf{W}=\mathcal{L}\left(\pi^{h} w\right)=\lim _{l \rightarrow \infty} \mathcal{L}\left(\left(\pi^{h} w\right)(l)\right)$. Here, $w(l)$ denotes the $l$ th dyadic piecewise linear approximation of $w$ and $\mathcal{L}$ denotes the rough path lift map defined by those dyadic approximations. By the explicit formula (5.6) for $\pi^{h} w$, we can easily see that $\pi^{h} \mathbf{W}$ is well defined a.s. with respect to $\mu$ and in fact equal to the Young translation of $\mathbf{W}$ by $-\sum_{l, l^{\prime}=1}^{n}\left\{\sigma\left[\psi_{1}\right](h)^{-1}\right\}_{l l^{\prime}}$. $g_{1}(w ; h)^{l} \cdot \sharp g_{1}(\cdot ; h)^{l^{\prime}}-\mathbf{i}_{h}\langle w\rangle$.

It is shown in [26] that $g_{2}$ actually has the following form: for some continuous $\operatorname{map} \hat{g}_{2}: G \Omega_{\alpha, 4 m}^{B}\left(\mathbb{R}^{d}\right) \times \mathcal{H} \rightarrow \mathcal{V}$ of 'quadratic order,'

$$
\begin{aligned}
g_{2}(w ; h) & =\hat{g}_{2}(\mathbf{W}, h) \\
& =\lim _{l \rightarrow \infty}\left\{\frac{1}{2} D^{2} \psi_{1}(h)\langle w(l), w(l)\rangle+\Pi_{\mathcal{V}} J_{1}(h) \int_{0}^{1} J_{s}(h)^{-1} V_{0}\left(\phi_{s}(h)\right) d s\right\} .
\end{aligned}
$$


Then, $g_{2}\left(\pi^{h} w ; h\right)=\hat{g}_{2}\left(\pi^{h} \mathbf{W}, h\right)$ is defined with respect to $\mu$. This in turn implies that $g_{2}(w ; h)=\hat{g}_{2}(\mathbf{W}, h)$ is well defined with respect to $\pi_{*}^{h} \mu$. Hence, if

$$
\begin{aligned}
& \int_{\mathcal{W}_{0}(h)} \exp \left(c_{1}\left\langle q(h), g_{2}(w ; h)\right\rangle_{\mathcal{V}}\right) \pi_{*}^{h} \mu(d w) \\
& \quad=\int_{\mathcal{W}} \exp \left(c_{1}\left\langle q(h), g_{2}\left(\pi^{h} w ; h\right)\right\rangle_{\mathcal{V}}\right) \mu(d w) \leqslant M
\end{aligned}
$$

holds for some $M>0$, then the proof of the lemma is done. (Since $g_{2}(w ; h)=$ $\hat{g}_{2}(\mathbf{W}, h)$ is $\infty$-quasicontinuous in $w$, the left hand side above is a constant multiple of the integral in (5.5).)

Now we show (5.7). It is straightforward to check that

$$
\mathbb{E}\left[D_{k}\left\langle q(h), g_{2}\left(\pi^{h} w ; h\right)\right\rangle_{\mathcal{V}}\right]=0 \quad \text { for all } k \in \mathcal{H}
$$

and $D^{3}\left\langle q(h), g_{2}\left(\pi^{h} w ; h\right)\right\rangle_{\mathcal{V}}=0$. This shows that

$$
\left\langle q(h), g_{2}\left(\pi^{h} w ; h\right)\right\rangle_{\mathcal{V}}-\mathbb{E}\left[\left\langle q(h), g_{2}\left(\pi^{h} w ; h\right)\right\rangle_{\mathcal{V}}\right]
$$

belongs to $\mathcal{C}_{2}$. The corresponding symmetric Hilbert-Schmidt bilinear form is easily calculated as

$$
\left(k, k^{\prime}\right) \mapsto \frac{1}{2} D_{k, k^{\prime}}^{2}\left\langle q(h), g_{2}\left(\pi^{h} w ; h\right)\right\rangle_{\mathcal{V}}=\frac{1}{2}\left\langle q(h), D^{2} \psi_{1}(h)\left\langle\pi^{h} k, \pi^{h} k^{\prime}\right\rangle\right\rangle_{\mathcal{V}}
$$

From (5.4) and boundedness of $\mathbb{E}\left[\left\langle q(h), g_{2}\left(\pi^{h} w ; h\right)\right\rangle_{\mathcal{V}}\right]$ in $h$, we obtain (5.7).

We give two technical lemmas (Lemmas 5.2 and 5.3) on integrability of certain Wiener functionals which will appear in the asymptotic expansion. Since the solution of RDE is involved in these lemmas, we assume for safety that the coefficients are of $C_{b}^{\infty}$. In the proofs of these lemmas we write $g_{j}=g_{j}(w ; h)$, $R_{j}^{\varepsilon}=R_{j}^{\varepsilon}(w ; h)$ for simplicity of notations.

The first one below corresponds to [47, Lemma 6.4]. Note that the constant $\gamma$ depends on $r$, but not on $h$ with $\|h\|_{\mathcal{H}} \leqslant r$.

LEMMA 5.2. Assume (A1), (B1)-(B3) and boundedness of $V_{i}(0 \leqslant i \leqslant r)$. Then, the following assertions (i) and (ii) hold:

(i) For any $r>0$ and $c>0$, there exists $\gamma>0$ such that

$$
\sup _{0<\varepsilon \leqslant 1} \sup _{\|h\|_{\mathcal{H}} \leqslant r} \mathbb{E}\left[\exp \left(c\left|\frac{R_{2}^{\varepsilon}(\cdot, h)}{\varepsilon^{2}}\right|\right) \mathbf{1}_{U_{\gamma}}(\varepsilon \mathbf{W})\right]<\infty .
$$

(ii) For any $r>0$ and $c>0$, there exists $\gamma>0$ such that

$$
\sup _{0<\varepsilon \leqslant 1} \sup _{\|h\|_{\mathcal{H}} \leqslant r} \mathbb{E}\left[\exp \left(c\left|\frac{R_{3}^{\varepsilon}(\cdot, h)}{\varepsilon^{2}}\right|\right) \mathbf{1}_{U_{\gamma}}(\varepsilon \mathbf{W})\right]<\infty .
$$


Proof. We use the deterministic estimates (3.31)-(3.34) for the Taylor-like expansion of the Lyons-Itô map and Besov-Hölder embedding for rough path spaces with $\alpha^{\prime}=\alpha-1 /(4 m)$. If $\varepsilon \mathbf{W} \in U_{\gamma^{\prime}}$, then

$$
\left|R_{j}^{\varepsilon}\right| \leqslant C\left(\varepsilon+\left\|(\varepsilon \mathbf{W})^{1}\right\|_{\alpha, 4 m}+\left\|(\varepsilon \mathbf{W})^{2}\right\|_{2 \alpha, 2 m}^{1 / 2}\right)^{j}
$$

for some positive constant $C=C\left(j, \gamma^{\prime}, r\right)$. We take $\gamma^{\prime}=1$ below. For $0<\gamma<1$,

$$
c\left|R_{2}^{\varepsilon} / \varepsilon\right|^{2} \leqslant c C(\varepsilon+\gamma)^{2}\left(1+\left\|\mathbf{W}^{1}\right\|_{\alpha, 4 m}+\left\|\mathbf{W}^{2}\right\|_{2 \alpha, 2 m}^{1 / 2}\right)^{2} .
$$

Hence, we have

$$
\begin{aligned}
& \sup _{0<\varepsilon \leqslant \gamma} \sup _{\|h\|_{\mathcal{H}} \leqslant r} \mathbb{E}\left[\exp \left(c\left|R_{2}^{\varepsilon} / \varepsilon\right|^{2}\right) \mathbf{1}_{U_{\gamma}(\varepsilon \mathbf{W})}\right] \\
& \quad \leqslant \mathbb{E}\left[\exp \left(4 c C \gamma^{2}\left(1+\left\|\mathbf{W}^{1}\right\|_{\alpha, 4 m}+\left\|\mathbf{W}^{2}\right\|_{2 \alpha, 2 m}^{1 / 2}\right)^{2}\right)\right] .
\end{aligned}
$$

By a Fernique-type theorem for Brownian rough path, the right hand side is integrable if $\gamma$ is chosen sufficiently small. Once $\gamma$ is fixed, we can easily estimate the integral for $\varepsilon \in[\gamma, 1]$. Thus, we have shown assertion (i). The proof of assertion (ii) is essentially the same.

The other one is a key technical lemma. It corresponds to [47, Lemma 6.5].

LEMMA 5.3. Assume (A1), (B1)-(B3) and boundedness of $V_{i}(0 \leqslant i \leqslant r)$. Let $c_{1}>1$ be as in (5.5) in Lemma 5.1. Then, for any $c_{2} \in\left(1, c_{1}\right)$, there exists a constant $\gamma_{1}>0$ which is independent of $h \in \mathcal{K}_{a}^{\min }$ and satisfies that

$$
\begin{aligned}
& \sup _{0<\varepsilon \leqslant 1} \sup _{h \in \mathcal{K}_{a}^{\min }} \mathbb{E}\left[\exp \left(c_{2}\left\langle q(h), R_{2}^{\varepsilon}(\cdot, h)\right\rangle_{\mathcal{V}} / \varepsilon^{2}\right) \mathbf{1}_{U_{\gamma_{1}}}(\varepsilon \mathbf{W}) ;\left|\mathbf{i}_{h}\right|^{2}\right. \\
& \left.\quad+\left|R_{1}^{\varepsilon}(\cdot, h) / \varepsilon\right|^{2} \leqslant \kappa^{2}\right]<\infty
\end{aligned}
$$

for any $\kappa>0$.

Proof. Thanks to Lemma 5.2(ii) and Hölder's inequality, it is sufficient to show the following integrability. For any $c_{2} \in\left(1, c_{1}\right)$, there exists $\gamma>0$ such that

$$
\sup _{0<\varepsilon \leqslant 1} \sup _{h \in \mathcal{K}_{a}^{\min }} \mathbb{E}\left[\exp \left(c_{2}\left\langle q(h), g_{2}\right\rangle_{\mathcal{V}}\right) \mathbf{1}_{U_{\gamma}}(\varepsilon \mathbf{W}) ;\left|\mathbf{i}_{h}\right|^{2}+\left|R_{1}^{\varepsilon} / \varepsilon\right|^{2} \leqslant \kappa^{2}\right]<\infty
$$

for any $\kappa>0$. 
By straightforward computation, we see that

$$
\begin{aligned}
g_{2}(w ; h)=\lim _{l \rightarrow \infty} g_{2}(w(l) ; h) \\
=\lim _{l \rightarrow \infty}\left\{\frac{1}{2} D^{2} \psi_{1}(h)\langle w(l), w(l)\rangle+\Pi_{\mathcal{V}} J_{1}(h) \int_{0}^{1} J_{s}(h)^{-1} V_{0}\left(\phi_{s}(h)\right) d s\right\} \\
=\lim _{l \rightarrow \infty}\left\{\frac{1}{2} D^{2} \psi_{1}(h)\left\langle\left(\pi^{h} w\right)(l),\left(\pi^{h} w\right)(l)\right\rangle\right. \\
\left.\quad+\Pi_{\mathcal{V}} J_{1}(h) \int_{0}^{1} J_{s}(h)^{-1} V_{0}\left(\phi_{s}(h)\right) d s\right\} \\
\quad+\lim _{l \rightarrow \infty}\left\{D^{2} \psi_{1}(h)\left\langle\left(\pi^{h} w\right)(l),\left(\left(\pi^{h}\right)^{\perp} w\right)(l)\right\rangle\right. \\
\left.\quad+\frac{1}{2} D^{2} \psi_{1}(h)\left\langle\left(\left(\pi^{h}\right)^{\perp} w\right)(l),\left(\left(\pi^{h}\right)^{\perp} w\right)(l)\right\rangle\right\} \\
=g_{2}\left(\pi^{h} w ; h\right)+D^{2} \psi_{1}(h)\left\langle\pi^{h} w,\left(\pi^{h}\right)^{\perp} w\right\rangle+\frac{1}{2} D^{2} \psi_{1}(h)\left\langle\left(\pi^{h}\right)^{\perp} w,\left(\pi^{h}\right)^{\perp} w\right\rangle .
\end{aligned}
$$

Here, we used basic properties of Young translation and the fact that $g_{2}(\cdot ; h)$ is actually a continuous function in $\mathbf{W}=\mathcal{L}(w)$.

There exists a positive constant $C$ (which depends only on $\|h\|_{\mathcal{H}}$ and may vary from line to line) such that

$$
\begin{aligned}
\left|g_{2}(w ; h)-g_{2}\left(\pi^{h} w ; h\right)\right| & \leqslant C\left(\left\|\pi^{h} w\right\|_{\mathcal{W}}\left\|\left(\pi^{h}\right)^{\perp} w\right\|_{\mathcal{H}}+\left\|\left(\pi^{h}\right)^{\perp} w\right\|_{\mathcal{H}}^{2}\right) \\
& \leqslant C\left(\|w\|_{\mathcal{W}}\left\|\left(\pi^{h}\right)^{\perp} w\right\|_{\mathcal{H}}+\left\|\left(\pi^{h}\right)^{\perp} w\right\|_{\mathcal{H}}^{2}\right) \\
& \leqslant C\left\{\frac{\rho^{2}}{2}\|w\|_{\mathcal{W}}^{2}+\left(\frac{1}{2 \rho^{2}}+2\right)\left\|\left(\pi^{h}\right)^{\perp} w\right\|_{\mathcal{H}}^{2}\right\}
\end{aligned}
$$

where $\rho>0$ is a small constant which will be determined later and we used the estimate for $D^{2} \phi_{1}$ given just below (4.2).

Under the condition that $\left|\mathbf{i}_{h}\right|^{2}+\left|R_{1}^{\varepsilon} / \varepsilon\right|^{2} \leqslant \kappa^{2}$, we can easily see that $\left|g_{1}(w ; h)\right| \leqslant\left|R_{1}^{\varepsilon} / \varepsilon-R_{2}^{\varepsilon} / \varepsilon\right| \leqslant \kappa+\left|R_{2}^{\varepsilon} / \varepsilon\right|$. From these, we have $\left\|\left(\pi^{h}\right)^{\perp} w\right\|_{\mathcal{H}} \leqslant C\left(\kappa+\left|R_{2}^{\varepsilon} / \varepsilon\right|\right)$ and

$$
\left|g_{2}(w ; h)-g_{2}\left(\pi^{h} w ; h\right)\right| \leqslant \frac{C \rho^{2}}{2}\|w\|_{\mathcal{W}}^{2}+C\left(\frac{1}{2 \rho^{2}}+2\right)\left(\kappa^{2}+\left|\frac{R_{2}^{\varepsilon}}{\varepsilon}\right|^{2}\right) .
$$

Note that $C>0$ is independent of $\kappa, \varepsilon, \rho, w, h$. 
Using Hölder's inequality with $p=c_{1} / c_{2} \in(1, \infty)$ and $1 / p+1 / p^{\prime}=1$, we have

$$
\begin{aligned}
& \mathbb{E}\left[\exp \left(c_{2}\left\langle q(h), g_{2}\right\rangle_{\mathcal{V}}\right) \mathbf{1}_{U_{\gamma}}(\varepsilon \mathbf{W}) ;\left|\mathbf{i}_{h}\right|^{2}+\left|R_{1}^{\varepsilon} / \varepsilon\right|^{2} \leqslant \kappa^{2}\right] \\
& \leqslant \mathbb{E}\left[\exp \left(c_{1}\left\langle q(h), g_{2} \circ \pi^{h}\right\rangle \mathcal{V}\right) \mathbf{1}_{U_{\gamma}}(\varepsilon \mathbf{W}) ;\left|\mathbf{i}_{h}\right|^{2}+\left|R_{1}^{\varepsilon} / \varepsilon\right|^{2} \leqslant \kappa^{2}\right]^{1 / p} \\
& \times \mathbb{E}\left[\exp \left(p^{\prime} c_{2} \max _{h \in \mathcal{K}_{a}^{\min }}\left|q(h) \| g_{2}-g_{2} \circ \pi^{h}\right|\right) \mathbf{1}_{U_{\gamma}}(\varepsilon \mathbf{W}) ;\left|\mathbf{i}_{h}\right|^{2}\right. \\
&\left.+\left|R_{1}^{\varepsilon} / \varepsilon\right|^{2} \leqslant \kappa^{2}\right]^{1 / p^{\prime}} \\
& \leqslant(2 \pi)^{\left(n+n^{\prime}\right) / 2 p}\left\{\operatorname{det} \sigma\left[\psi_{1}\right](h)\right\}^{1 / 2 p} \mathbb{E}\left[\exp \left(c_{1}\left\langle q(h), g_{2}\right\rangle \mathcal{V}\right) \delta_{0}\left(g_{1}, \mathbf{i}_{h}\right)\right]^{1 / p} \\
& \times \mathbb{E}\left[\exp \left(\frac{C^{\prime} \rho^{2}}{2}\|w\|_{\mathcal{W}}^{2}+C^{\prime}\left(\frac{1}{2 \rho^{2}}+2\right)\left(\kappa^{2}+\left|\frac{R_{2}^{\varepsilon}}{\varepsilon}\right|^{2}\right)\right) \mathbf{1}_{U_{\gamma}}(\varepsilon \mathbf{W})\right]^{1 / p^{\prime}},
\end{aligned}
$$

where we set $C^{\prime}=C p^{\prime} c_{2} \max _{h \in \mathcal{K}_{a}^{\min }}|q(h)|$. The first factor on the right hand side of (5.10) above is dominated by a positive constant independent of $h \in \mathcal{K}_{a}^{\text {min }}$, due to (5.5) or (5.7). Now, we choose $\rho$ so small that $\exp \left(C^{\prime} \rho^{2}\|w\|_{\mathcal{W}}^{2}\right)$ is integrable, which is possible by Fernique's theorem, and use Schwarz' inequality for the second factor. Then, by Lemma 5.2(i), we can choose $\gamma>0$ so that $\sup _{\varepsilon} \sup _{h}$ of the second factor is finite. Thus, we have shown (5.8), which completes the proof.

COROLlARY 5.4. Keep the same notations and assumptions as in Lemma 5.3. Then, the following (i)-(iii) hold:

(i) For any $c_{2} \in\left(1, c_{1}\right)$ and any $\kappa>0$,

$$
\sup _{h \in \mathcal{K}_{a}^{\min }} \mathbb{E}\left[\exp \left(c_{2}\left\langle q(h), g_{2}(\cdot, h)\right\rangle_{\mathcal{V}}\right) ;\left|\mathbf{i}_{h}\right|^{2}+\left|g_{1}(\cdot, h)\right|^{2} \leqslant \kappa^{2}\right]<\infty .
$$

(ii) For any smooth function $f$ on $\mathcal{V} \times T_{h} \mathcal{K}_{a}^{\text {min }}$ with compact support,

$$
\exp \left(c_{2}\left\langle q(h), g_{2}(\cdot, h)\right\rangle_{\mathcal{V}}\right) f\left(g_{1}(\cdot, h), \mathbf{i}_{h}\right) \in \tilde{\mathbb{D}}_{\infty} .
$$

Moreover, as $h$ varies in $\mathcal{K}_{a}^{\min }$, these Wiener functionals form a bounded set in $\tilde{\mathbb{D}}_{\infty}$.

(iii) Let $T \in \mathcal{S}^{\prime}\left(\mathcal{V} \times T_{h} \mathcal{K}_{a}^{\min }\right)$ with compact support. Take any two smooth function $f$ and $\hat{f}$ on $\mathcal{V} \times T_{h} \mathcal{K}_{a}^{\min }$ with compact support such that $f \equiv 1 \equiv \hat{f}$ on the support of $T$. Then,

$$
\begin{aligned}
& \exp \left(c_{2}\left\langle q(h), g_{2}(\cdot, h)\right\rangle_{\mathcal{V}}\right) f\left(g_{1}(\cdot, h), \mathbf{i}_{h}\right) T\left(g_{1}(\cdot, h), \mathbf{i}_{h}\right) \\
& \quad=\exp \left(c_{2}\left\langle q(h), g_{2}(\cdot, h)\right\rangle_{\mathcal{V}}\right) \hat{f}\left(g_{1}(\cdot, h), \mathbf{i}_{h}\right) T\left(g_{1}(\cdot, h), \mathbf{i}_{h}\right) \in \mathbb{D}_{-\infty} .
\end{aligned}
$$


In particular, the generalized expectations of these two Watanabe distributions also coincide. Moreover, as $h$ varies in $\mathcal{K}_{a}^{\min }$, these Watanabe distributions form a bounded set in $\mathbb{D}_{-\infty}$.

Proof. We can easily prove (i) by applying Fatou's lemma to the inequality in Lemma 5.3. Noting that

$$
\mathbf{1}_{\left\{\left|\mathbf{i}_{h}\right|^{2}+\left|g_{1}(\cdot, h)\right|^{2} \leqslant(\kappa / 2)^{2}\right\}} \leqslant \liminf _{\varepsilon \searrow 0} \mathbf{1}_{\left\{\left|\mathbf{i}_{h}\right|^{2}+\left|R_{1}^{\varepsilon}(\cdot, h) / \varepsilon\right|^{2} \leqslant \kappa^{2}\right\}}
$$

for almost all $w$, we have the desired inequality with $\kappa$ being replaced by $\kappa / 2$. Since, $\kappa>0$ is arbitrary, we have shown (i).

It is straightforward to check (ii) from (i). We now prove (iii). Since $T\left(g_{1}(\cdot, h), \mathbf{i}_{h}\right)$ is a well-defined element in $\tilde{\mathbb{D}}_{-\infty}$, we see from (ii) that both sides belong to $\mathbb{D}_{-\infty}$. To check the equality, we just have to use $f \cdot T=T=\hat{f} \cdot T$ as finite-dimensional distributions.

REMARK 5.5. We may and will write $\exp \left(c_{2}\left\langle q(h), g_{2}(\cdot, h)\right\rangle_{\mathcal{V}}\right) T\left(g_{1}(\cdot, h), \mathbf{i}_{h}\right) \in$ $\mathbb{D}_{-\infty}$ for simplicity in the situation of Corollary 5.4(iii). Later, we usually choose $T$ to be a partial derivative of the delta function. Note that $\operatorname{since} \exp \left(c_{2}\langle q(h)\right.$, $\left.\left.g_{2}(\cdot, h)\right\rangle_{\mathcal{V}}\right) \notin \tilde{\mathbb{D}}_{\infty}$, the product $\exp \left(c_{2}\left\langle q(h), g_{2}(\cdot, h)\right\rangle_{\mathcal{V}}\right) \Phi$ or its generalized expectation cannot be defined for a general element $\Phi \in \tilde{\mathbb{D}}_{-\infty}$.

\section{Asymptotic partition of unity}

In this section, by using rough path theory, we modify the argument on an asymptotic partition of unity in [47, Section 6.6].

Let $f: \mathbb{R} \rightarrow \mathbb{R}$ be an even smooth function such that $f(s)=1$ if $|s| \leqslant 1 / 2$ and $f(s)=0$ if $|s| \geqslant 1$. We also assume that $f$ is nonincreasing on $[0, \infty)$. Define, for $\gamma>0, \varepsilon \in(0,1]$ and $h \in \mathcal{H}$,

$$
\begin{gathered}
\zeta_{\gamma}^{\varepsilon}(w)=f\left(\frac{\left\|(\varepsilon \mathbf{W})^{1}\right\|_{\alpha, 4 m-B}^{4 m}+\left\|(\varepsilon \mathbf{W})^{2}\right\|_{2 \alpha, 2 m-B}^{2 m}}{\gamma^{4 m}}\right) \\
\zeta_{\gamma}^{\varepsilon, h}(w)=\zeta_{\gamma}^{\varepsilon}\left(w-\frac{h}{\varepsilon}\right)=f\left(\frac{\left\|\tau_{-h}(\varepsilon \mathbf{W})^{1}\right\|_{\alpha, 4 m-B}^{4 m}+\left\|\tau_{-h}(\varepsilon \mathbf{W})^{2}\right\|_{2 \alpha, 2 m-B}^{2 m}}{\gamma^{4 m}}\right) .
\end{gathered}
$$

Here, $\tau_{-h}$ stands for the Young translation by $-h$ on the geometric rough path space. Note that these are $\mathbb{D}_{\infty}$-functionals. It is clear that (i) $\zeta_{\gamma}^{\varepsilon}=0$ if $\varepsilon \mathbf{W} \notin U_{\gamma}$ and $\zeta_{\gamma}^{\varepsilon, h}=0$ if $\varepsilon \mathbf{W} \notin U_{h, \gamma}$ and (ii) $\zeta_{\gamma}^{\varepsilon}=1$ if $\varepsilon \mathbf{W} \in U_{\gamma}^{\prime}$ and $\zeta_{\gamma}^{\varepsilon, h}=1$ if $\varepsilon \mathbf{W} \in U_{h, \gamma}^{\prime}$. 
For $h_{1}, \ldots, h_{N} \in \mathcal{K}_{a}^{\min }$ and $\gamma_{1}, \ldots, \gamma_{N}>0$ satisfying that $\mathcal{L}\left(\mathcal{K}_{a}^{\min }\right) \subset$ $\bigcup_{v=1}^{N} U_{h_{v}, \gamma_{v}}^{\prime}$, we define

$$
\begin{gathered}
\chi^{\varepsilon}(w)=1-\prod_{\nu=1}^{N}\left(1-\zeta_{\gamma_{v}}^{\varepsilon, h_{v}}(w)\right), \\
\tilde{\chi}_{v}^{\varepsilon}(w)=\zeta_{\gamma_{v}}^{\varepsilon, h_{v}}(w) \prod_{\mu=1}^{\nu-1}\left(1-\zeta_{\gamma_{\mu}}^{\varepsilon, h_{\mu}}(w)\right)
\end{gathered}
$$

for $1 \leqslant v \leqslant N$. Loosely speaking, $\chi^{\varepsilon}(w)=1$ means that $\varepsilon \mathbf{w}$ is close to $\mathbf{h}_{v}$ for some $v$. Similarly, $\tilde{\chi}_{v}^{\varepsilon}(w)=1$ loosely means that $\varepsilon \mathbf{w}$ is close to $\mathbf{h}_{v}$, but distant from $\mathbf{h}_{1}, \ldots, \mathbf{h}_{v-1}$. It is clear that $0 \leqslant \chi^{\varepsilon} \leqslant 1$ and easy to see that $\sum_{v=1}^{N} \tilde{\chi}_{v}^{\varepsilon}=\chi^{\varepsilon}$.

It is easy to see that

$$
\zeta_{\gamma_{v}}^{\varepsilon, h_{v}}\left(w+\frac{k}{\varepsilon}\right)=1+O\left(\varepsilon^{l}\right) \quad \text { in } \mathbb{D}_{\infty} \text { as } \varepsilon \searrow 0 \text { for any } l>0
$$

uniformly in $k \in \mathcal{L}^{-1}\left(U_{h_{v}, \gamma_{v}}^{\prime}\right) \cap \mathcal{K}_{a}^{\min }$. (Note that the Wiener functional on the left hand is the composition of $f$ and a polynomial in $\varepsilon$ with the coefficients from an inhomogeneous Wiener chaos. Therefore, it clearly has an asymptotic expansion in $\mathbb{D}_{\infty}$-topology and we just need to check that the coefficients vanish by formal differentiation.) In a similar way, we have

$$
\chi^{\varepsilon}\left(w+\frac{k}{\varepsilon}\right)=1+O\left(\varepsilon^{l}\right) \quad \text { in } \mathbb{D}_{\infty} \text { as } \varepsilon \searrow 0 \text { for any } l>0
$$

uniformly in $k \in \mathcal{K}_{a}^{\min }$. Note that we have repeatedly used the fact that $f(x)=1$ on $[-1 / 2,1 / 2]$.

Now we get back to our SDE/RDE and choose such $N, h_{v}$ and $\gamma_{v}(1 \leqslant v \leqslant N)$. Before going into details, we explain below why our problem is reduced to the case where $V_{i}(0 \leqslant i \leqslant r)$ is of $C_{b}^{\infty}$. (This remark is unnecessary if $V_{i}$ is of $C_{b}^{\infty}$ in the first place.)

REMARK 6.1. Since the deterministic Itô map is continuous under (A1),

$$
R:=\sup \left\{\left|x+\Phi\left(\mathbf{h}, \lambda^{\varepsilon}\right)_{0, t}^{1}\right| \mid t \in[0,1], \varepsilon \in[0,1], h \in \mathcal{K}_{a}^{\min }\right\} \in(0, \infty) .
$$

From a result in [2] (or a standard cut-off argument, alternatively) we see the following: there exists a neighborhood $\Lambda$ of $\mathcal{L}\left(\mathcal{K}_{a}^{\min }\right)$ in $G \Omega_{\alpha, 4 m-B}^{B}\left(\mathbb{R}^{r}\right)$ such that

$$
\sup \left\{\left|x+\Phi\left(\mathbf{w}, \lambda^{\varepsilon}\right)_{0, t}^{1}\right| \mid t \in[0,1], \varepsilon \in[0,1], \mathbf{w} \in \Lambda\right\} \leqslant 2 R .
$$


Therefore, if we work on the neighborhood, we only need information of $V_{i}$ restricted to the ball of radius $2 R$.

In the argument in the rest of this section, taking $\gamma=\gamma_{h}>0$ smaller if necessary, we have $U_{h, \gamma} \subset \Lambda$. For these reasons, we may and will assume in the sequel that $V_{i}$ is of $C_{b}^{\infty}$.

(When we throw away contributions from the complement set of a neighborhood of $\mathcal{L}\left(\mathcal{K}_{a}^{\mathrm{min}}\right)$ in Lemma 7.1 below, we use the upper bound estimate of the LDP in Theorem 3.8. Hence, it is important that Theorem 3.8 holds under (A1).)

For any $h \in \mathcal{K}_{a}^{\min }$, there exists a coordinate neighborhood $O_{h}$ of $h$ (with respect to $\mathcal{H}$-topology) and a constant $\gamma=\gamma_{h}>0$ such that the following conditions holds:

$$
\mathcal{K}_{a}^{\min } \cap U_{h, \gamma}=\left\{k \in \mathcal{K}_{a}^{\min } \mid k \in U_{h, \gamma}\right\} \subset O_{h} .
$$

For any $\varepsilon \in(0,1], \int_{O_{h}} D^{(k)}(\varepsilon w) \Delta^{(k)}(\varepsilon w) \omega(d k)=1 \quad$ on $\left\{w \in W \mid \varepsilon \mathbf{W} \in U_{h, \gamma}\right\}$.

$$
\begin{aligned}
& \sup _{k \in O_{h}}\left(\sum_{j=1}^{n^{\prime}}\left|\left(\frac{\partial \iota}{\partial \theta^{j}}\right)_{k}\right|_{\mathcal{H}}+\sum_{j, j^{\prime}=1}^{n^{\prime}}\left|\left(\frac{\partial^{2} \iota}{\partial \theta^{j} \partial \theta^{j^{\prime}}}\right)_{k}\right|_{\mathcal{H}}\right)<\infty \\
& \text { and } \inf _{k \in O_{h}} \operatorname{det} G(k)>0 .
\end{aligned}
$$

Here, $\left(\theta^{1}, \ldots, \theta^{n^{\prime}}\right)$ is the local coordinate on $O_{h}$. Note that (6.4) and (6.5) are immediate from Lemma 4.6 and Remark 4.7. (Note that $\gamma$ above can be taken smaller if necessary. See Remark 4.7.)

We choose any $1<c_{2}<c_{1}$ as in Lemmas 5.1 and 5.3 and fix them in what follows. Let $\lambda>0, \gamma_{0}>0$ and $\varepsilon_{0} \in(0,1]$ be the constants defined in (3.36) and Lemma 3.7, respectively. Similarly, let $\gamma_{1}>0$ be the constant that appeared in Lemma 5.3.

We write $\rho=\rho_{h}:=\sup _{k \in O_{h}}\|k-h\|_{\mathcal{H}}>0$. Then, we can take $O_{h}$ and $\gamma=$ $\gamma_{h}>0$ so small that the following inequalities hold:

$$
\begin{gathered}
\sigma=\sigma_{h}:=\frac{16^{1 / 4 m} C \rho}{\gamma} \geqslant 2^{1 / 4 m}, \\
2\left(1+32^{1 / 4 m}\right)^{4 m} C^{4 m} \rho^{4 m}=2\left(2^{1 / 4 m} \sigma \gamma+C \rho\right)^{4 m} \leqslant \gamma_{0}^{4 m}, \\
2(\gamma+C \rho)^{4 m} \leqslant \gamma_{1}^{4 m} .
\end{gathered}
$$


Here, $C=C_{\alpha, 4 m}>0$ is the smallest constant that satisfies the estimates (3.23)(3.24). It is easy to check that such $\rho$ and $\gamma$ exist. First, take $O_{h}$ (or $\rho$ ) small enough and fix it and then we can retake $\gamma>0$ smaller if necessary for this fixed $\rho$.

Note that the condition (6.8) immediately implies the following: if $\varepsilon \mathbf{W} \in$ $U_{h-k, 2^{1 / 4 m} \sigma \gamma}$, that is,

$$
\left\|\tau_{k-h}(\varepsilon \mathbf{W})^{1}\right\|_{\alpha, 4 m-B}^{4 m}+\left\|\tau_{k-h}(\varepsilon \mathbf{W})^{2}\right\|_{2 \alpha, 2 m-B}^{2 m} \leqslant 2 \sigma^{4 m} \gamma^{4 m}
$$

holds for $k \in O_{h}$, then $\left\|(\varepsilon \mathbf{W})^{1}\right\|_{\alpha, 4 m-B}^{4 m}+\left\|(\varepsilon \mathbf{W})^{2}\right\|_{2 \alpha, 2 m-B}^{2 m} \leqslant \gamma_{0}^{4 m}$, because of (3.23), (3.24), (6.8) and the obvious fact that $\varepsilon \mathbf{W}=\tau_{-k+h} \circ \tau_{k-h}(\varepsilon \mathbf{W})$. (Thus, the assumption of Lemma 3.7 is satisfied.) Therefore, under (6.10) we have

$$
\begin{aligned}
& \inf _{k \in O_{h}} \inf _{z:|z|=1} z^{*} \sigma\left[\left(R_{1}^{\varepsilon}(\cdot ; k) / \varepsilon, \mathbf{i}_{k}\right)\right](w) z \geqslant \frac{\lambda \wedge 1}{2} \\
& \quad \text { if } \varepsilon \in\left(0, \varepsilon_{0}\right] \text { and } \varepsilon \mathbf{W} \in U_{h-k, 2^{1 / 4 m} \sigma \gamma} .
\end{aligned}
$$

Likewise, if $\left\|\tau_{k-h}(\varepsilon \mathbf{W})^{1}\right\|_{\alpha, 4 m-B}^{4 m}+\left\|\tau_{k-h}(\varepsilon \mathbf{W})^{2}\right\|_{2 \alpha, 2 m-B}^{2 m} \leqslant \gamma^{4 m}$ for $k \in O_{h}$, then (3.23), (3.24) and (6.9) imply that $\left\|(\varepsilon \mathbf{W})^{1}\right\|_{\alpha, 4 m-B}^{4 m}+\left\|(\varepsilon \mathbf{W})^{2}\right\|_{2 \alpha, 2 m-B}^{2 m} \leqslant \gamma_{1}^{4 m}$. In other words, $U_{h-k, \gamma} \subset U_{\gamma_{1}}$ for $k \in O_{h}$. Therefore, by Lemma 5.3,

$$
\begin{aligned}
& \sup _{0<\varepsilon \leqslant 1} \sup _{k \in O_{h}} \mathbb{E}\left[\exp \left(\frac{c_{2}}{\varepsilon^{2}}\left\langle q(k), R_{2}^{\varepsilon}(w ; k)\right\rangle_{\mathcal{V}}\right) \mathbf{1}_{U_{h-k, \gamma}}(\varepsilon \mathbf{W}) ;\left|\mathbf{i}_{k}\right|^{2}\right. \\
& \left.+\left|\frac{R_{1}^{\varepsilon}(w ; k)}{\varepsilon}\right|^{2} \leqslant \kappa^{2}\right]<\infty
\end{aligned}
$$

for any $\kappa>0$.

Thus, we have shown the following lemma:

LEMma 6.2. Assume (A1) and (B1)-(B3). For any $h \in \mathcal{K}_{a}^{\min }$, we can find $O_{h}$ and $\gamma_{h}$ such that (6.4), (6.5), (6.6), (6.11), (6.12) hold.

Since $\mathcal{K}_{a}^{\min }$ is compact, there exist finitely many $h_{1}, \ldots, h_{N} \in \mathcal{K}_{a}^{\min }$ and $\gamma_{1}, \ldots$, $\gamma_{N}>0$ such that $\mathcal{L}\left(\mathcal{K}_{a}^{\min }\right) \subset \bigcup_{v=1}^{N} U_{h_{v}, \gamma_{v}}^{\prime}$, where we wrote $\gamma_{v}=\gamma_{h_{v}}$ for simplicity $(1 \leqslant v \leqslant N)$. In the next section we denote by $\chi^{\varepsilon}$ and $\tilde{\chi}_{v}^{\varepsilon}$ the asymptotic partition of unity defined in (6.1), (6.2) associated with these data $\left\{\left(h_{v}, \gamma_{v}\right)\right\}_{v=1}^{N}$.

REMARK 6.3. The definitions of $\chi^{\varepsilon}$ and $\tilde{\chi}_{v}^{\varepsilon}$ in (6.1) and (6.2) depend only on the data $N$ and $\left\{\left(h_{v}, \gamma_{v}\right)\right\}_{v=1}^{N}$. Information of the SDE is involved only in choosing such data. Therefore, our version of the asymptotic partition of unity is much simpler than the one in [47] and looks more powerful. This is one of the advantages of our rough path approach. (See [47, Section 6.6] and compare.) 


\section{Proof of the main theorem: the Euclidean case}

In this section we prove our main theorem in the Euclidean setting (Theorem 2.2). We assume (A1)-(A2), (B1)-(B3) and (C1) throughout this section. We set $G^{\varepsilon}(w)=G(\varepsilon, w)$ below. Define

$$
\mathbb{E}\left[G^{\varepsilon} \delta_{a}\left(Y_{1}^{\varepsilon}\right)\right]=\mathbb{E}\left[\left(1-\chi^{\varepsilon}\right) G^{\varepsilon} \delta_{a}\left(Y_{1}^{\varepsilon}\right)\right]+\mathbb{E}\left[\chi^{\varepsilon} G^{\varepsilon} \delta_{a}\left(Y_{1}^{\varepsilon}\right)\right]=: I_{1}(\varepsilon)+I_{2}(\varepsilon) .
$$

Due to the large deviation upper bound (Theorem 3.8), the first term $I_{1}(\varepsilon)$ does not contribute to the asymptotic expansion.

LEMMA 7.1. There exist positive constants $c, C$ such that

$$
\left|I_{1}(\varepsilon)\right| \leqslant C \exp \left(-\frac{d_{a}^{2}+c}{2 \varepsilon^{2}}\right) \text { as } \varepsilon \searrow 0 .
$$

Here, $d_{a}=\inf \left\{\|h\|_{\mathcal{H}} \mid \psi(1, x, h)=a\right\}>0$.

Proof. We use Theorem 3.8. By the way of construction, $1-\chi^{\varepsilon}$ is dominated by $\mathbf{1}_{\left\{\varepsilon \mathbf{W} \in O^{c}\right\}}$ for some open set $O \subset G \Omega_{\alpha, 4 m}^{B}\left(\mathbb{R}^{d}\right)$ containing $\mathcal{K}_{a}^{\min }$. Therefore, we have

$$
\left|I_{1}(\varepsilon)\right|=\left|\int_{\mathcal{W}}\left(1-\chi^{\varepsilon}\right) \tilde{G}^{\varepsilon} \theta_{a}^{\varepsilon}(d w)\right| \leqslant \mu_{a}^{\varepsilon}\left(O^{c}\right)^{1 / p}\left\{\int_{\mathcal{W}}\left|\tilde{G}^{\varepsilon}\right|^{p^{\prime}} \theta_{a}^{\varepsilon}(d w)\right\}^{1 / p^{\prime}}
$$

for any $p, p^{\prime} \in(1, \infty)$ with $1 / p+1 / p^{\prime}=1$. Here, $\tilde{G}^{\varepsilon}$ is an $\infty$-quasicontinuous modification of $G^{\varepsilon}$.

Since $O^{c}$ is closed and the rate function $I$ is good, it is easy to see that $\inf _{\mathbf{w} \in O^{c}} I(\mathbf{w})>d_{a}^{2} / 2$. Set $\kappa:=\left(\inf _{\mathbf{w} \in O^{c}} I(\mathbf{w})-d_{a}^{2} / 2\right) / 4>0$. By Theorem 3.8 we have

$$
\begin{aligned}
\mu_{a}^{\varepsilon}\left(O^{c}\right)^{1 / p} & =O\left(\exp \left(\frac{-\inf _{\mathbf{w} \in O^{c}} I(\mathbf{w})+\kappa}{p \varepsilon^{2}}\right)\right) \\
& \leqslant O\left(\exp \left(\frac{-\inf _{\mathbf{w} \in O^{c}} I(\mathbf{w})+2 \kappa}{\varepsilon^{2}}\right)\right)
\end{aligned}
$$

as $\varepsilon \searrow 0$ if $p>1$ is sufficiently close to 1 .

On the other hand, when $p^{\prime}=2 l$ with arbitrarily large $l \in \mathbb{N}$,

$$
\int_{\mathcal{W}}\left|\tilde{G}^{\varepsilon}\right|^{2 l} \theta_{a}^{\varepsilon}(d w)=\mathbb{E}\left[\left(G^{\varepsilon}\right)^{2 l} \delta_{a}\left(Y_{1}^{\varepsilon}\right)\right] \leqslant\left\|\left(G^{\varepsilon}\right)^{2 l}\right\|_{j, 2}\left\|\delta_{a}\left(Y_{1}^{\varepsilon}\right)\right\|_{-j, 2}
$$

for some $j \in \mathbb{N}$ such that $\delta_{a}\left(Y_{1}^{\varepsilon}\right)$ belongs to the Sobolev space $\mathbb{D}_{-j, 2}$. ( $j$ is independent of $l$.) Moreover, it is well known that $\left\|\delta_{a}\left(Y_{1}^{\varepsilon}\right)\right\|_{-j, 2}=O\left(\varepsilon^{-\nu}\right)$ as 
$\varepsilon \searrow 0$ for some $v>0$. (This comes from (A2) and the integration by parts formula.) Since we assumed in $(\mathbf{C 1})$ that $G^{\varepsilon}$ is bounded in $\mathbb{D}_{\infty},\left(G^{\varepsilon}\right)^{2 l}$ is bounded in $\mathbb{D}_{j, 2}$ for any $l, j$. Combining these all, we obtain

$$
\left|I_{1}(\varepsilon)\right|=O\left(\exp \left(\frac{-\inf _{\mathbf{w} \in O^{c}} I(\mathbf{w})+3 \kappa}{\varepsilon^{2}}\right)\right)
$$

as $\varepsilon \searrow 0$. If we set $c=2 \kappa$ this is the statement of the lemma.

Hence, the problem reduces to the asymptotic expansion for

$$
I_{2}(\varepsilon)=\sum_{\nu=1}^{N} \mathbb{E}\left[\tilde{\chi}_{\nu}^{\varepsilon} G^{\varepsilon} \delta_{a}\left(Y_{1}^{\varepsilon}\right)\right] .
$$

For the reason we stated in Remark 6.1, we may additionally assume that $V_{i}(0 \leqslant i \leqslant r)$ is bounded. Now we compute each summand.

Take $\eta \in C_{0}^{\infty}(\mathcal{V}, \mathbb{R})$ with $\eta \geqslant 0$ and $\int_{\mathcal{V}} \eta(y) d y=1$ and set $\eta_{l}(y)=l^{n} \eta(l y)$ for $l \geqslant 1$. Then, $\eta_{l}\left(Y_{1}^{\varepsilon}-a\right) \rightarrow \delta_{a}\left(Y_{1}^{\varepsilon}\right)$ as $l \rightarrow \infty$ in $\tilde{\mathbb{D}}_{-\infty}$. Noting that $\tilde{\chi}_{v}^{\varepsilon}(w)=$ $\tilde{\chi}_{v}^{\varepsilon}(w) \mathbf{1}_{\left\{\varepsilon \mathbf{W} \in U_{h_{v}, \gamma_{v}}\right\}}$ by definition, we have from (6.5) that

$$
\begin{aligned}
\mathbb{E}\left[\tilde{\chi}_{v}^{\varepsilon} G^{\varepsilon} \cdot \eta_{l}\left(Y_{1}^{\varepsilon}-a\right)\right] & =\mathbb{E}[\tilde{\chi}_{v}^{\varepsilon} G^{\varepsilon} \cdot \eta_{l}\left(Y_{1}^{\varepsilon}-a\right) \cdot \underbrace{\int_{O_{v}} D^{(k)}(\varepsilon w) \Delta^{(k)}(\varepsilon w) \omega(d k)}_{=1}] \\
& =\int_{O_{v}} \mathbb{E}\left[\tilde{\chi}_{v}^{\varepsilon} G^{\varepsilon} \cdot \eta_{l}\left(Y_{1}^{\varepsilon}-a\right) \cdot D^{(k)}(\varepsilon w) \Delta^{(k)}(\varepsilon w)\right] \omega(d k),
\end{aligned}
$$

where we set $O_{v}=O_{h_{v}}$.

Let $\left(\theta^{1}, \ldots, \theta^{n^{\prime}}\right)$ be the local coordinate on $O_{v}$ and let $\hat{O}_{v} \subset \mathbb{R}^{n^{\prime}}$ be the image of $O_{v}$. As before $k \in O_{v}$ is denoted by $k(\theta), \theta \in \hat{O}_{v}$. Set $G(\theta)=G(k(\theta))$ and $\left[e_{\beta}(\theta)\right]_{\beta=1}^{n^{\prime}}=G(\theta)^{-1 / 2}\left[\left(\partial k / \partial \theta^{\beta}\right)(\theta)\right]_{\beta=1}^{n^{\prime}}$. Then, $\left\{e_{\beta}(\theta)\right\}_{\beta=1}^{n^{\prime}}$ is an orthonormal basis of $T_{k(\theta)} \mathcal{K}_{a}^{\min } \subset \mathcal{H}$. We set $\mathbf{e}(\theta, w)=\left[\left\langle e_{\beta}(\theta), w\right\rangle\right]_{\beta=1}^{n^{\prime}}$, which is a continuous linear map from $\mathcal{W}$ to $\mathbb{R}^{n^{\prime}}$. (Recall that $\mathbf{i}_{k(\theta)}\langle w\rangle=\sum_{\beta=1}^{n^{\prime}}\left\langle e_{\beta}(\theta), w\right\rangle e_{\beta}(\theta)$, which is almost the same as $\mathbf{e}(\theta, w)$, but it takes values in $T_{k(\theta)} \mathcal{K}_{a}^{\min }$.) By definition we have $D^{(k(\theta))}(\varepsilon w) \Delta^{(k(\theta))}(\varepsilon w)=\operatorname{det}\left[-\varepsilon \mathbf{a}_{k(\theta)}(w)\right] \delta_{0}(\varepsilon \mathbf{e}(\theta, w))$.

Combining these with the Cameron-Martin translation by $k(\theta) / \varepsilon$, we see that

$$
\begin{aligned}
\mathbb{E} & {\left[\tilde{\chi}_{v}^{\varepsilon} G^{\varepsilon} \eta_{l}\left(Y_{1}^{\varepsilon}-a\right)\right] } \\
& =\int_{\hat{O}_{v}} \mathbb{E}\left[\tilde{\chi}_{v}^{\varepsilon} G^{\varepsilon} \eta_{l}\left(Y_{1}^{\varepsilon}-a\right) \operatorname{det}\left[-\varepsilon \mathbf{a}_{k(\theta)}\right] \delta_{0}(\varepsilon \mathbf{e}(\theta, \cdot))\right] \sqrt{\operatorname{det} G(\theta)} d \theta
\end{aligned}
$$




$$
\begin{aligned}
= & \int_{\hat{O}_{v}} \mathbb{E}\left[\exp \left(-\frac{1}{\varepsilon}\langle k(\theta), w\rangle_{\mathcal{H}}-\frac{1}{2 \varepsilon^{2}}|k(\theta)|_{\mathcal{H}}^{2}\right) \tilde{\chi}_{v}^{\varepsilon}\left(w+\frac{k(\theta)}{\varepsilon}\right)\right. \\
& \times G\left(\varepsilon, w+\frac{k(\theta)}{\varepsilon}\right) \eta_{l}\left(Y_{1}^{\varepsilon, k(\theta)}-a\right) \\
& \left.\times \operatorname{det}\left[-\varepsilon \mathbf{a}_{k(\theta)}(w)-\mathbf{a}_{k(\theta)}(k(\theta))\right] \delta_{0}(\varepsilon \mathbf{e}(\theta, w)+\mathbf{e}(\theta, k(\theta)))\right] \sqrt{\operatorname{det} G(\theta)} d \theta \\
= & \exp \left(-\frac{d_{a}^{2}}{2 \varepsilon^{2}}\right) \int_{\hat{O}_{v}} \mathbb{E}\left[e^{-\langle k(\theta), w\rangle_{\mathcal{H}} / \varepsilon} \tilde{\chi}_{v}^{\varepsilon}\left(w+\frac{k(\theta)}{\varepsilon}\right) G\left(\varepsilon, w+\frac{k(\theta)}{\varepsilon}\right)\right. \\
& \left.\times \eta_{l}\left(R_{1}^{\varepsilon}(w ; k(\theta))\right) \operatorname{det}\left[\operatorname{Id}_{n^{\prime}}-\varepsilon \mathbf{a}_{k(\theta)}(w)\right] \zeta_{\sigma_{v} \gamma_{v}}^{\varepsilon, h_{v}}\left(w+\frac{k(\theta)}{\varepsilon}\right) \delta_{0}(\varepsilon \mathbf{e}(\theta, w))\right] \\
& \times \sqrt{\operatorname{det} G(\theta)} d \theta .
\end{aligned}
$$

Here, we used the following facts: (i) $\mathbf{e}(\theta, k(\theta))=0$, (ii) $\mathbf{a}_{k(\theta)}(k(\theta))=-\operatorname{Id}_{n^{\prime}}$ by (3.37), and (iii) $\tilde{\chi}_{v}^{\varepsilon}(w+k(\theta) / \varepsilon)=\tilde{\chi}_{v}^{\varepsilon}(w+k(\theta) / \varepsilon) \zeta_{\sigma_{v} \gamma_{v}}^{\varepsilon, h_{v}}(w+k(\theta) / \varepsilon)$, which is immediate from $f(s)=f(s) f\left(s / \sigma_{v}^{4 m}\right)$ for all $s \in \mathbb{R}$, due to (6.7). (Recall that $f$, $\zeta_{\gamma}^{\varepsilon, h}$ and $\tilde{\chi}_{v}^{\varepsilon}$ were introduced at the beginning of Section 6.)

Now we apply Proposition 3.1 with $F(w)=\left(R_{1}^{\varepsilon}(w ; k(\theta)) / \varepsilon, \mathbf{e}(\theta, w)\right), \chi=f$,

$$
\xi(w)=\xi_{\varepsilon}^{(v)}(w):=\frac{\left\|\tau_{k(\theta)-h_{v}}(\varepsilon \mathbf{W})^{1}\right\|_{\alpha, 4 m-B}^{4 m}+\left\|\tau_{k(\theta)-h_{v}}(\varepsilon \mathbf{W})^{2}\right\|_{2 \alpha, 2 m-B}^{2 m}}{\sigma_{v}^{4 m} \gamma_{v}^{4 m}} .
$$

Note that $\xi(w)$ is just a polynomial of order $4 m$ and $\chi(\xi)=\zeta_{\sigma_{v} \gamma_{v}}^{\varepsilon, h_{v}}(w+k(\theta) / \varepsilon)$, which is on the right hand side of (7.3). As we have seen in (6.10) and (6.11), $|\xi(w)| \leqslant 2$ implies that the smallest eigenvalue of $\sigma[F]$ is greater than or equal to $(\lambda \wedge 1) / 2$. Hence, we can use Proposition 3.1 to obtain that, for each $0<\varepsilon \leqslant \varepsilon_{0}$,

$$
\begin{aligned}
& \lim _{l \rightarrow \infty} \zeta_{\sigma_{\nu} \gamma_{\nu}}^{\varepsilon, h_{\nu}}\left(w+\frac{k(\theta)}{\varepsilon}\right) \eta_{l}\left(R_{1}^{\varepsilon}(w ; k(\theta))\right) \delta_{0}(\varepsilon \mathbf{e}(\theta, w)) \\
& =\zeta_{\sigma_{v} \gamma_{\nu}}^{\varepsilon, h_{\nu}}\left(w+\frac{k(\theta)}{\varepsilon}\right) \delta_{0}\left(R_{1}^{\varepsilon}(w ; k(\theta)), \varepsilon \mathbf{e}(\theta, w)\right) \text { in } \tilde{\mathbb{D}}_{-\infty}
\end{aligned}
$$

uniformly in $\theta \in \hat{O}_{v}$. Here, the delta function on the right hand side is defined on $\mathcal{V} \times \mathbb{R}^{n^{\prime}}$.

Therefore, we have

$$
\begin{aligned}
\mathbb{E}\left[\tilde{\chi}_{v}^{\varepsilon} G^{\varepsilon} \delta_{a}\left(Y_{1}^{\varepsilon}\right)\right] & =e^{-d_{a}^{2} / 2 \varepsilon^{2}} \int_{\hat{O}_{v}} \mathbb{E}\left[e^{-\langle k(\theta), w\rangle_{\mathcal{H}} / \varepsilon} \tilde{\chi}_{\nu}^{\varepsilon}\left(w+\frac{k(\theta)}{\varepsilon}\right) G\left(\varepsilon, w+\frac{k(\theta)}{\varepsilon}\right)\right. \\
& \left.\times \operatorname{det}\left[\operatorname{Id}-\varepsilon \mathbf{a}_{k(\theta)}(w)\right] \zeta_{\sigma_{v} \gamma_{v}}^{\varepsilon, h_{v}}\left(w+\frac{k(\theta)}{\varepsilon}\right) \delta_{0}\left(R_{1}^{\varepsilon}(w ; k(\theta)), \varepsilon \mathbf{e}(\theta, w)\right)\right]
\end{aligned}
$$




$$
\begin{aligned}
& \times \sqrt{\operatorname{det} G(\theta)} d \theta \\
= & \varepsilon^{-\left(n+n^{\prime}\right)} e^{-d_{a}^{2} / 2 \varepsilon^{2}} \int_{\hat{O}_{v}} \mathbb{E}\left[e^{-\langle k(\theta), w\rangle_{\mathcal{H}} / \varepsilon} \tilde{\chi}_{v}^{\varepsilon}\left(w+\frac{k(\theta)}{\varepsilon}\right) G\left(\varepsilon, w+\frac{k(\theta)}{\varepsilon}\right)\right. \\
& \left.\times \operatorname{det}\left[\operatorname{Id}-\varepsilon \mathbf{a}_{k(\theta)}(w)\right] \zeta_{\sigma_{v} \gamma_{\nu}}^{\varepsilon, h_{v}}\left(w+\frac{k(\theta)}{\varepsilon}\right) \delta_{0}\left(\frac{R_{1}^{\varepsilon}(w ; k(\theta))}{\varepsilon}, \mathbf{e}(\theta, w)\right)\right] \\
& \times \sqrt{\operatorname{det} G(\theta)} d \theta \\
= & \varepsilon^{-\left(n+n^{\prime}\right)} e^{-d_{a}^{2} / 2 \varepsilon^{2}} \int_{O_{v}} \mathbb{E}\left[e^{-\langle k, w\rangle_{\mathcal{H}} / \varepsilon} \tilde{\chi}_{v}^{\varepsilon}\left(w+\frac{k}{\varepsilon}\right) G\left(\varepsilon, w+\frac{k}{\varepsilon}\right)\right. \\
& \left.\times \operatorname{det}\left[\operatorname{Id}-\varepsilon \mathbf{a}_{k}(w)\right] \zeta_{\sigma_{v} \gamma_{v}}^{\varepsilon, h_{v}}\left(w+\frac{k}{\varepsilon}\right) \delta_{0}\left(\frac{R_{1}^{\varepsilon}(w ; k)}{\varepsilon}, \mathbf{i}_{k}\langle w\rangle\right)\right] \omega(d k),
\end{aligned}
$$

where in the last line $\delta_{0}$ stands for the delta function on $\mathcal{V} \times T_{k} \mathcal{K}_{a}^{\text {min }}$.

Under the condition that $R_{1}^{\varepsilon}(w ; k)=0,\langle k, w\rangle_{\mathcal{H}}=\left\langle q(k), D \psi_{1}(k)\langle w\rangle\right\rangle_{\mathcal{V}}=$ $\left\langle q(k), g_{1}(w ; k)\right\rangle_{\mathcal{V}}$ is equal to $-\left\langle q(k), R_{2}^{\varepsilon}(w ; k)\right\rangle_{\mathcal{V}} / \varepsilon$.

Since $f\left(\left(|y|^{2}+|z|^{2}\right) / \kappa^{2}\right) \delta_{0}(y, z)=\delta_{0}(y, z)$ as a distribution on $\mathcal{V} \times T_{k} \mathcal{K}_{a}^{\text {min }}$ for any $\kappa>0$, where $(y, z)$ denotes a generic element in $\mathcal{V} \times T_{k} \mathcal{K}_{a}^{\text {min }}$, we can see that

$$
\begin{gathered}
\zeta_{\sigma_{\nu} \gamma_{\nu}}^{\varepsilon, h_{\nu}}\left(w+\frac{k}{\varepsilon}\right) f\left(\frac{\kappa^{\varepsilon}(w ; k)}{\kappa^{2}}\right) \delta_{0}\left(\frac{R_{1}^{\varepsilon}(w ; k)}{\varepsilon}, \mathbf{i}_{k}\langle w\rangle\right) \\
=\zeta_{\sigma_{\nu} \gamma_{\nu}}^{\varepsilon, h_{\nu}}\left(w+\frac{k}{\varepsilon}\right) \delta_{0}\left(\frac{R_{1}^{\varepsilon}(w ; k)}{\varepsilon}, \mathbf{i}_{k}\langle w\rangle\right)
\end{gathered}
$$

in $\tilde{\mathbb{D}}_{-\infty}$, where we set $\kappa^{\varepsilon}(w ; k)=\left|R_{1}^{\varepsilon}(w ; k) / \varepsilon\right|^{2}+\left|\mathbf{i}_{k}\langle w\rangle\right|^{2}$ for simplicity.

Summing up these all, we have the following lemma:

LEMMA 7.2. For any $1 \leqslant v \leqslant N, 0 \leqslant \varepsilon \leqslant \varepsilon_{0}, \kappa>0$, it holds that

$$
\begin{aligned}
\mathbb{E}\left[\tilde{\chi}_{\nu}^{\varepsilon} G^{\varepsilon} \delta_{a}\left(Y_{1}^{\varepsilon}\right)\right]= & \varepsilon^{-\left(n+n^{\prime}\right)} e^{-d_{a}^{2} / 2 \varepsilon^{2}} \\
& \times \int_{O_{v}} \mathbb{E}\left[e^{\left\langle q(k), R_{2}^{\varepsilon}(w ; k) / \varepsilon^{2}\right\rangle} \tilde{\chi}_{\nu}^{\varepsilon}\left(w+\frac{k}{\varepsilon}\right) G\left(\varepsilon, w+\frac{k}{\varepsilon}\right)\right. \\
& \times \operatorname{det}\left[\operatorname{Id}-\varepsilon \mathbf{a}_{k}(w)\right] f\left(\frac{\kappa^{\varepsilon}(w ; k)}{\kappa^{2}}\right) \zeta_{\sigma_{v} \gamma_{v}}^{\varepsilon, h_{v}}\left(w+\frac{k}{\varepsilon}\right) \\
& \left.\times \delta_{0}\left(\frac{R_{1}^{\varepsilon}(w ; k)}{\varepsilon}, \mathbf{i}_{k}\langle w\rangle\right)\right] \omega(d k) .
\end{aligned}
$$

Here, $\varepsilon_{0} \in(0,1]$ is the constant given in Lemma 3.7 .

Now it suffices to compute a full asymptotic expansion of the generalized expectation in Lemma 7.2 above. We write $A_{v}(\varepsilon, k):=\mathbb{E}[\cdots]$ on the right hand 
side in (7.4). Note that all the asymptotics below are uniform in $k \in \mathcal{K}_{a}^{\min }$. So, we do not explicitly write uniformity or boundedness in $k$.

Now we list the asymptotic expansion of each factor in (7.4). First, by Assumption (C1),

$$
G\left(\varepsilon, w+\frac{k}{\varepsilon}\right) \sim \Gamma_{0}(k)+\varepsilon \Gamma_{1}(w ; k)+\varepsilon^{2} \Gamma_{2}(w ; k)+\cdots \quad \text { in } \mathbb{D}_{\infty} \text { as } \varepsilon \searrow 0 .
$$

Next, $\operatorname{det}\left[\operatorname{Id}-\varepsilon \mathbf{a}_{k}(w)\right]$ is just a polynomial:

$$
\operatorname{det}\left[\operatorname{Id}-\varepsilon \mathbf{a}_{k}(w)\right]=\mathcal{J}_{0}(w ; k)+\varepsilon \mathcal{J}_{1}(w ; k)+\cdots+\varepsilon^{n^{\prime}} \mathcal{J}_{n^{\prime}}(w ; k)
$$

for some $\mathcal{J}_{j}(w ; k) \in \mathbb{D}_{\infty}\left(1 \leqslant j \leqslant n^{\prime}\right)$. Note that $\mathcal{J}_{0}(w ; k) \equiv 1$.

From (3.23), (3.24) and the definition of $\sigma_{v}$ in (6.7), we can easily see that

$$
0 \leqslant \lim _{\varepsilon \searrow 0} \xi_{\varepsilon}(w)=\frac{\left\|\mathcal{L}\left(k-h_{v}\right)^{1}\right\|_{\alpha, 4 m-B}^{4 m}+\left\|\mathcal{L}\left(k-h_{v}\right)^{2}\right\|_{2 \alpha, 2 m-B}^{2 m}}{\sigma_{v}^{4 m} \gamma_{v}^{4 m}} \leqslant \frac{1}{8},
$$

which is one of the assumptions in Proposition 3.2. It is clear that

$$
\begin{aligned}
\left(R_{1}^{\varepsilon}(w ; k) / \varepsilon, \mathbf{i}_{k}\langle w\rangle\right) \sim & \left(g_{1}(w ; k), \mathbf{i}_{k}\langle w\rangle\right) \\
& +\varepsilon\left(g_{2}(w ; k), 0\right)+\cdots \quad \text { in } \mathbb{D}_{\infty}\left(\mathbb{R}^{n+n^{\prime}}\right) \text { as } \varepsilon \searrow 0 .
\end{aligned}
$$

Hence, we can use Proposition 3.2 to obtain

$$
\begin{aligned}
& \zeta_{\sigma_{v} \gamma_{\nu}}^{\varepsilon, h_{\nu}}\left(w+\frac{k}{\varepsilon}\right) \delta_{0}\left(\frac{R_{1}^{\varepsilon}(w ; k)}{\varepsilon}, \mathbf{i}_{k}\langle w\rangle\right) \\
& \quad \sim \Phi_{0}(w ; k)+\varepsilon \Phi_{1}(w ; k)+\varepsilon^{2} \Phi_{2}(w ; k)+\cdots \quad \text { in } \tilde{\mathbb{D}}_{-\infty} \text { as } \varepsilon \searrow 0
\end{aligned}
$$

The coefficient $\Phi_{j}(w ; k)$ is obtained by the formal asymptotic expansion for the composition of $\delta_{0}$ and $\left(R_{1}^{\varepsilon}(w ; k) / \varepsilon, \mathbf{i}_{k}\langle w\rangle\right)$ as explained in Proposition 3.2. In particular, $\Phi_{0}(w ; k)=\delta_{0}\left(g_{1}(w ; k), \mathbf{i}_{k}\langle w\rangle\right)$.

Let $L \in \mathbb{N}$ be arbitrarily large. Since $e^{\left\langle q(k), R_{2}^{\varepsilon}(w ; k) / \varepsilon^{2}\right\rangle} \tilde{\chi}_{\nu}^{\varepsilon}(w+k / \varepsilon) f\left(\kappa^{\varepsilon}(w ; k) / \kappa^{2}\right)$ is bounded in $\tilde{\mathbb{D}}_{\infty}$ due to Lemma 5.3 , we have

$$
\begin{aligned}
A_{v}(\varepsilon, k)= & \mathbb{E}\left[e^{\left\langle q(k), R_{2}^{\varepsilon}(w ; k) / \varepsilon^{2}\right\rangle} \tilde{\chi}_{\nu}^{\varepsilon}\left(w+\frac{k}{\varepsilon}\right) G\left(\varepsilon, w+\frac{k}{\varepsilon}\right)\right. \\
& \left.\times \operatorname{det}\left[\operatorname{Id}-\varepsilon \mathbf{a}_{k}(w)\right] f\left(\frac{\kappa^{\varepsilon}(w ; k)}{\kappa^{2}}\right)\left(\sum_{j=0}^{L} \varepsilon^{j} \Phi_{j}(w ; k)\right)\right]+O\left(\varepsilon^{L+1}\right)
\end{aligned}
$$

as $\varepsilon \searrow 0$. 
Then, we expand $e^{\left\langle q(k), R_{2}^{\varepsilon}(w ; k) / \varepsilon^{2}\right\rangle} \mathcal{\chi} \tilde{\chi}_{\nu}^{\varepsilon}(w+k / \varepsilon) f\left(\kappa^{\varepsilon}(w ; k) / \kappa^{2}\right)$ in $\tilde{\mathbb{D}}_{\infty}$ by using Lemma 5.3. It is clear that

$$
\begin{aligned}
\frac{R_{3}^{\varepsilon}(w ; k)}{\varepsilon^{2}}= & \frac{R_{2}^{\varepsilon}(w ; k)}{\varepsilon^{2}}-g_{2}(w ; k) \sim \varepsilon g_{3}(w ; k)+\varepsilon^{2} g_{4}(w ; k)+\cdots \quad \text { in } \mathbb{D}_{\infty}\left(\mathbb{R}^{n}\right) \\
& \text { as } \varepsilon \searrow 0 .
\end{aligned}
$$

In particular, $R_{3}^{\varepsilon}(w ; k) / \varepsilon^{2}=O(\varepsilon)$ in $\mathbb{D}_{\infty}\left(\mathbb{R}^{n}\right)$ as $\varepsilon \searrow 0$. From the Taylor expansion of $e^{x}$ at $x=0$, we have

$$
\begin{aligned}
\tilde{\chi}_{\nu}^{\varepsilon}\left(w+\frac{k}{\varepsilon}\right) f\left(\frac{\kappa^{\varepsilon}(w ; k)}{\kappa^{2}}\right) e^{\left\langle q(k), R_{2}^{\varepsilon}(w ; k) / \varepsilon^{2}\right\rangle_{\mathcal{V}}} \\
=\tilde{\chi}_{\nu}^{\varepsilon}\left(w+\frac{k}{\varepsilon}\right) f\left(\frac{\kappa^{\varepsilon}(w ; k)}{\kappa^{2}}\right)\left\{e^{\left\langle q(k), g_{2}(w ; k)\right\rangle \mathcal{V}} \sum_{j=0}^{L} \frac{\left\langle q(k), R_{3}^{\varepsilon}(w ; k) / \varepsilon^{2}\right\rangle_{\mathcal{V}}^{j}}{j !}\right. \\
\quad+\int_{0}^{1} d \tau \exp \left(\left\langle q(k),(1-\tau) g_{2}(w ; k)+\tau \frac{R_{2}^{\varepsilon}(w ; k)}{\varepsilon^{2}}\right\rangle_{\mathcal{V}}\right) \\
\left.\quad \times \frac{\left\langle q(k), R_{3}^{\varepsilon}(w ; k) / \varepsilon^{2}\right\rangle_{\mathcal{V}}^{L+1}}{(L+1) !}\right\}
\end{aligned}
$$

Using Lemma 5.3, (5.8) in its proof and Hölder's inequality with the exponents $1 / \tau$ and $1 /(1-\tau)$, we can show that the last term on the right hand side of (7.9) is $O\left(\varepsilon^{L+1}\right)$ in $\tilde{\mathbb{D}}_{\infty}$-topology. Here, we have used (6.12). Similarly, $e^{\left\langle q(k), g_{2}(w ; k)\right\rangle \nu} \tilde{\chi}_{\nu}^{\varepsilon}(w+k / \varepsilon) f\left(\kappa^{\varepsilon}(w ; k) / \kappa^{2}\right) \in \tilde{\mathbb{D}}_{\infty}$, due to (5.8).

Denote by $\mathcal{B}_{j}(w ; k) \in \mathbb{D}_{\infty}(j \in \mathbb{N})$ the coefficients that appear in the formal asymptotic expansion of

$$
\begin{aligned}
\sum_{j=0}^{\infty} \frac{\left\langle q(k), R_{3}^{\varepsilon}(w ; k) / \varepsilon^{2}\right\rangle_{\mathcal{V}}^{j}}{j !} \sim \mathcal{B}_{0}(w ; k)+\varepsilon \mathcal{B}_{1}(w ; k)+\varepsilon^{2} \mathcal{B}_{2}(w ; k)+\cdots \quad \text { in } \mathbb{D}_{\infty} \\
\quad \text { as } \varepsilon \searrow 0 .
\end{aligned}
$$

Note that $\mathcal{B}_{0}(w ; k) \equiv 1$. As $\varepsilon \searrow 0$, it holds that

$$
\begin{aligned}
\tilde{\chi}_{\nu}^{\varepsilon}\left(w+\frac{k}{\varepsilon}\right) f\left(\frac{\kappa^{\varepsilon}(w ; k)}{\kappa^{2}}\right) e^{\left\langle q(k), R_{2}^{\varepsilon}(w ; k) / \varepsilon^{2}\right\rangle \mathcal{\nu}} \\
=\tilde{\chi}_{\nu}^{\varepsilon}\left(w+\frac{k}{\varepsilon}\right) f\left(\frac{\kappa^{\varepsilon}(w ; k)}{\kappa^{2}}\right) e^{\left\langle q(k), g_{2}(w ; k)\right\rangle_{\mathcal{V}}} \\
\quad \times \sum_{j=0}^{L} \varepsilon^{j} \mathcal{B}_{j}(w ; k)+O\left(\varepsilon^{L+1}\right) \text { in } \tilde{\mathbb{D}}_{\infty}
\end{aligned}
$$


and therefore

$$
\begin{aligned}
A_{v}(\varepsilon, k)= & \mathbb{E}\left[e^{\left\langle q(k), g_{2}(w ; k)\right\rangle} \tilde{\chi}_{\nu}^{\varepsilon}\left(w+\frac{k}{\varepsilon}\right) G\left(\varepsilon, w+\frac{k}{\varepsilon}\right) \operatorname{det}\left[\operatorname{Id}-\varepsilon \mathbf{a}_{k}(w)\right]\right. \\
& \left.\times f\left(\frac{\kappa^{\varepsilon}(w ; k)}{\kappa^{2}}\right)\left(\sum_{j_{1}=0}^{L} \varepsilon^{j_{1}} \mathcal{B}_{j_{1}}(w ; k)\right)\left(\sum_{j_{2}=0}^{L} \varepsilon^{j_{2}} \Phi_{j_{2}}(w ; k)\right)\right] \\
& +O\left(\varepsilon^{L+1}\right) .
\end{aligned}
$$

By Proposition 3.2, each $\Phi_{j}(w ; k)$ is a finite sum of terms of the following form;

$$
\left(\mathrm{a} \mathbb{D}_{\infty} \text {-functional }\right) \times\left(\partial^{\beta} \delta_{0}\right)\left(g_{1}(w ; k), \mathbf{i}_{k}\langle w\rangle\right),
$$

where $\beta$ is a certain $\left(n+n^{\prime}\right)$-dimensional multi-index. Hence, by Corollary 5.4, (iii) and Remark 5.5, $\exp \left(\left\langle q(k), g_{2}(w ; k)\right\rangle_{\mathcal{V}}\right) \Phi_{j}(w ; k) \in \mathbb{D}_{-\infty}$. Note that all the other factors in the generalized expectation in (7.11) belong to $\mathbb{D}_{\infty}$. (Because $\exp \left(\left\langle q(k), g_{2}(w ; k)\right\rangle_{\mathcal{V}}\right) \notin \tilde{\mathbb{D}}_{\infty}$, a little care was needed above.) So, it suffices to expand them in $\mathbb{D}_{\infty}$-topology.

By the general theory, $f\left(\kappa^{\varepsilon}(w ; k) / \kappa^{2}\right)$ admits asymptotic expansion in $\mathbb{D}_{\infty^{-}}$topology as follows:

$$
\begin{aligned}
f\left(\frac{\kappa^{\varepsilon}(w ; k)}{\kappa^{2}}\right)= & f\left(\frac{\left|R_{1}^{\varepsilon}(w ; k) / \varepsilon\right|^{2}+\left|\mathbf{i}_{k}\langle w\rangle\right|^{2}}{\kappa^{2}}\right) \\
\sim & \mathcal{C}_{0}(w ; k)+\varepsilon \mathcal{C}_{1}(w ; k) \\
& +\varepsilon^{2} \mathcal{C}_{2}(w ; k)+\cdots \quad \text { in } \mathbb{D}_{\infty} \text { as } \varepsilon \searrow 0 .
\end{aligned}
$$

Note that $\mathcal{C}_{0}=f\left(\left\{\left|g_{1}(w ; k)\right|^{2}+\left|\mathbf{i}_{k}\langle w\rangle\right|^{2}\right\} / \kappa^{2}\right)$.

Let us see that $\left\{\mathcal{C}_{i}(w ; k)\right\}$ does not contribute to the asymptotic expansion. In other words, $f\left(\kappa^{\varepsilon}(w ; k) / \kappa^{2}\right)$ is merely a dummy factor which is introduced for technical reasons. Each term in $\mathcal{C}_{i}(w ; k)$ has a factor of the form $f^{(l)}\left(\left\{\left|g_{1}(w ; k)\right|^{2}+\left|\mathbf{i}_{k}\langle w\rangle\right|^{2}\right\} / \kappa^{2}\right)$ for some $l \in \mathbb{N}$. Similarly, by Proposition 3.2, each term of $\Phi_{j}(w ; k)$ has a factor of the form $\left(\partial^{\beta} \delta_{0}\right)\left(g_{1}(w ; k), \mathbf{i}_{k}\langle w\rangle\right)$ for some $\left(n+n^{\prime}\right)$-dimensional multi-index $\beta$. Noting that

$$
\begin{aligned}
& f^{(l)}\left(\frac{\left|g_{1}(w ; k)\right|^{2}+\left|\mathbf{i}_{k}\langle w\rangle\right|^{2}}{\kappa^{2}}\right) \cdot\left(\partial^{\beta} \delta_{0}\right)\left(g_{1}(w ; k), \mathbf{i}_{k}\langle w\rangle\right) \\
& \quad= \begin{cases}0 & (\text { if } l \geqslant 1), \\
\left(\partial^{\beta} \delta_{0}\right)\left(g_{1}(w ; k), \mathbf{i}_{k}\langle w\rangle\right) & (\text { if } l=0),\end{cases}
\end{aligned}
$$

we can easily see that $\mathcal{C}_{i}(w ; k) \Phi_{j}(w ; k)=0$ in $\tilde{\mathbb{D}}_{-\infty}$ if $i \geqslant 1$ and hence

$$
\sum_{i=0}^{\infty} \varepsilon^{i} \mathcal{C}_{i}(w ; k) \sum_{j=0}^{\infty} \varepsilon^{j} \Phi_{j}(w ; k)=\sum_{j=0}^{\infty} \varepsilon^{j} \Phi_{j}(w ; k)
$$

holds as a formal asymptotic expansion. 
Again by the general theory, $\tilde{\chi}_{v}^{\varepsilon}(w+k / \varepsilon)$ also admits an asymptotic expansion in $\mathbb{D}_{\infty}$ :

$$
\begin{aligned}
\tilde{\chi}_{\nu}^{\varepsilon}\left(w+\frac{k}{\varepsilon}\right) \sim & \mathcal{D}_{0}^{(v)}(w ; k)+\varepsilon \mathcal{D}_{1}^{(v)}(w ; k)+\varepsilon^{2} \mathcal{D}_{2}^{(v)}(w ; k)+\cdots \quad \text { in } \mathbb{D}_{\infty} \\
& \text { as } \varepsilon \searrow 0 .
\end{aligned}
$$

However, we should note here that the coefficient $\left\{\mathcal{D}_{i}^{(v)}(w ; k)\right\}$ may depend on $v$. If $k \notin O_{v}$, then $k \notin U_{h_{v}, \gamma_{v}}$, which implies that $\mathcal{D}_{i}^{(v)}(w ; k) \equiv 0$ for all $i \geqslant 0$. Moreover, since $\sum_{v=1}^{N} \tilde{\chi}_{v}^{\varepsilon}=\chi^{\varepsilon}$ and $\chi^{\varepsilon}$ admits the asymptotic expansion (6.3), we easily see that, for all $k \in \mathcal{K}_{a}^{\min }, \sum_{v} \mathcal{D}_{i}^{(v)}(w ; k) \equiv 0$ for all $i \geqslant 1$ and $\sum_{v} \mathcal{D}_{0}^{(v)}(w ; k) \equiv 1$.

We now set a notation. Define $\mathcal{E}_{i}(w ; k) \in \tilde{\mathbb{D}}_{-\infty}$ by the following formal asymptotic series:

$$
\begin{aligned}
\sum_{j=0}^{\infty} \varepsilon^{j} \mathcal{E}_{j}(w ; k) & \\
= & \left(\sum_{j_{1}=0}^{\infty} \varepsilon^{j_{1}} \Gamma_{j_{1}}(w ; k)\right)\left(\sum_{j_{2}=0}^{m} \varepsilon^{j_{2}} \mathcal{J}_{j_{2}}(w ; k)\right)\left(\sum_{j_{3}=0}^{\infty} \varepsilon^{j_{3}} \mathcal{B}_{j_{3}}(w ; k)\right) \\
& \times\left(\sum_{j_{4}=0}^{\infty} \varepsilon^{j_{4}} \Phi_{j_{4}}(w ; k)\right) .
\end{aligned}
$$

Note that $\Gamma_{j}(w ; k), \mathcal{J}_{j}(w ; k), \mathcal{B}_{j}(w ; k), \Phi_{j}(w ; k)$ are all defined not just for $k \in$ $O_{v}$, but for any $k \in \mathcal{K}_{a}^{\text {min }}$. Hence, so is $\mathcal{E}_{j}(w ; k)$, which means that the definition of $\mathcal{E}_{j}(w ; k)$ is independent of $v$. It is easy to see that $\mathcal{E}_{0}(w ; k)=\Gamma_{0}(k) \delta_{0}\left(g_{1}(w ; k)\right.$, $\left.\mathbf{i}_{k}\langle w\rangle\right)$.

Combining these all, we have the following asymptotics: for any $L \in \mathbb{N}$,

$$
A_{\nu}(\varepsilon, k)=\mathbb{E}\left[e^{\left\langle q(k), g_{2}(w ; k)\right\rangle \mathcal{\nu}}\left(\sum_{j=0}^{L} \varepsilon^{j} \mathcal{E}_{j}(w ; k)\right)\left(\sum_{l=0}^{L} \varepsilon^{l} \mathcal{D}_{l}^{(\nu)}(w ; k)\right)\right]+O\left(\varepsilon^{L+1}\right)
$$

as $\varepsilon \searrow 0$ uniformly in $k \in \mathcal{K}_{a}^{\min }$. Therefore,

$$
\begin{aligned}
& \sum_{\nu=1}^{N} \mathbb{E}\left[\tilde{\chi}_{\nu}^{\varepsilon} G^{\varepsilon} \delta_{a}\left(Y_{1}^{\varepsilon}\right)\right] \\
& \quad=\sum_{\nu=1}^{N}\left\{\int_{O_{\nu}} \mathbb{E}\left[e^{\left\langle q(k), g_{2}(w ; k)\right\rangle \nu}\left(\sum_{j=0}^{L} \varepsilon^{j} \mathcal{E}_{j}(w ; k)\right)\left(\sum_{l=0}^{L} \varepsilon^{l} \mathcal{D}_{l}^{(v)}(w ; k)\right)\right] \omega(d k)\right.
\end{aligned}
$$




$$
\begin{aligned}
& \left.+O\left(\varepsilon^{L+1}\right)\right\} \\
& =\sum_{\nu=1}^{N}\left\{\int_{\mathcal{K}_{a}^{\min }} \mathbb{E}\left[e^{\left\langle q(k), g_{2}(w ; k)\right\rangle}\left(\sum_{j=0}^{L} \varepsilon^{j} \mathcal{E}_{j}(w ; k)\right)\left(\sum_{l=0}^{L} \varepsilon^{l} \mathcal{D}_{l}^{(v)}(w ; k)\right)\right] \omega(d k)\right. \\
& \left.+O\left(\varepsilon^{L+1}\right)\right\} \\
& =\sum_{j=0}^{L} \varepsilon^{j} \int_{\mathcal{K}_{a}^{\min }} \mathbb{E}\left[e^{\left\langle q(k), g_{2}(w ; k)\right\rangle} \mathcal{E}_{j}(w ; k)\right] \omega(d k)+O\left(\varepsilon^{L+1}\right)
\end{aligned}
$$

as $\varepsilon \searrow 0$, which is the desired asymptotic expansion. Note that $\left\{\mathcal{D}_{i}^{(v)}(w ; k)\right\}$ does not contribute to the asymptotic expansion, either. The leading term is given by

$$
c_{0}=\int_{\mathcal{K}_{a}^{\min }} \mathbb{E}\left[e^{\left\langle q(k), g_{2}(w ; k)\right\rangle \mathcal{v}} \delta_{0}\left(g_{1}(w ; k), \mathbf{i}_{k}\langle w\rangle\right)\right] \Gamma_{0}(k) \omega(d k),
$$

which is positive if $\Gamma_{0}$ is non-negative, but not identically zero on $\mathcal{K}_{a}^{\min }$.

To complete the proof of our main theorem (Theorem 2.2), we show that $c_{2 j+1}=0(j=0,1,2, \ldots)$ under $(\mathbf{C 2})$. First, note that $f_{2 j}(w ; h)$ and $f_{2 j+1}(w ; h)$ are even and odd in $w$, respectively. (For simplicity, we say that $\left\{f_{j}\right\}$ satisfies the even-odd property.) Obviously, so does $\left\{g_{j}\right\}$. (See (3.29) and (3.30) for the definitions of $\left\{f_{j}\right\}$ and $\left\{g_{j}\right\}$.) It immediately follows from this that $\left\{\mathcal{B}_{j}\right\}$ satisfies this property. Although not so obvious, it is still straightforward to check the evenodd property of $\left\{\Phi_{j}\right\}$. From the explicit form of $\mathbf{a}_{k}(\varepsilon w)$, it is easy to see that $\left\{\mathcal{J}_{j}\right\}$ has the same property, too. Therefore, $\left\{\mathcal{E}_{j}\right\}$ satisfies the even-odd property and

$$
\mathbb{E}\left[e^{\left\langle q(k), g_{2}(w ; k)\right\rangle \mathcal{V}} \mathcal{E}_{2 j+1}(w ; k)\right]=0
$$

by the invariance of the Wiener measure $\mu$ and $g_{2}(w ; k)$ under $w \mapsto-w$. (Although it is not necessary in the proof of this Euclidean case, we note that $\left\{\mathcal{D}_{j}^{(v)}\right\}$ also satisfies this property due to the explicit construction of $\tilde{\chi}_{v}^{\varepsilon}$.) Thus, we have shown Theorem 2.2.

\section{Proof of the manifold case}

In this section we prove Theorem 2.5. As we mentioned before, we choose Riemannian metrics on $\mathcal{M}$ and $\mathcal{N}$ arbitrarily and fix them. The measure vol is the Riemannian measure on $\mathcal{N}$. It is often useful to embed $\mathcal{M}$ into $\mathbb{R}^{N}$ with sufficiently high dimension $N$ and solve differential equations in the ambient space $\mathbb{R}^{N}$. For instance, when we prove continuity or differentiability of various maps from (rough) path spaces, this extrinsic view has an advantage. 
Choose such an embedding $\mathcal{M} \hookrightarrow \mathbb{R}^{N}$ and extend $V_{i}(0 \leqslant i \leqslant r)$ so that it becomes $C_{b}^{\infty}$-vector field on $\mathbb{R}^{N}$. (By abusing notations we denote the extended vector fields by the same symbols.) Then, consider Stratonovich SDE (2.1) and skeleton ODE (2.2) on $\mathbb{R}^{N}$. If $x \in \mathcal{M}$, then the solutions stay in $\mathcal{M}$ and coincide with the solutions (2.6) and (2.7), respectively.

The case of RDE is slightly different. We do not define RDE on $\mathcal{M}$ in an intrinsic way. We just solve RDE (3.17) on $\mathbb{R}^{N}$ and call the first level path of the solution the solution of RDE on $\mathcal{M}$. (Note that if $x \in \mathcal{M}$, then the first level path of the solution stays in $\mathcal{M}$.) Since we only need the first level path of the solution of the RDE, this is enough for our purpose.

Recall that $J(t, x, h)$ defined by (3.10) on the ambient space is the Jacobian of the smooth map $x \mapsto \phi(t, x, w)$. Hence, $J(t, x, h)$ is a linear map from $T_{x}\left(\mathbb{R}^{N}\right)$ to $T_{\phi(t, x, h)}\left(\mathbb{R}^{N}\right)$. When restricted to $T_{x}(\mathcal{M})$, it is from $T_{x}(\mathcal{M})$ to $T_{\phi(t, x, h)}(\mathcal{M})$ and coincides with the Jacobian of $x \mapsto \phi(t, x, w)$ defined by (2.7) on $\mathcal{M}$. A similar fact holds for $X^{\varepsilon}(t, x, w)$ and $J^{\varepsilon}(t, x, w)$.

We remark that all the results in Section 4 remain valid in the manifold setting with trivial modifications. To see this, let us write key quantities in an intrinsic way. As usual we identify $\mathcal{H}$ and $\mathcal{H}^{*}$ via the Riesz isometry. We denote by $\mathbb{L}(\mathcal{X}, \mathcal{Y})$ the space of bounded linear maps from $\mathcal{X}$ to $\mathcal{Y}$.

First, $D \phi_{t}(h) \in \mathbb{L}\left(\mathcal{H}, T_{\phi_{t}(h)} \mathcal{M}\right)$ and

$$
D \psi_{t}(h)=\left(\Pi_{*}\right)_{\phi_{t}(h)} \circ D \phi_{t}(h) \in \mathbb{L}\left(\mathcal{H}, T_{\psi_{t}(h)} \mathcal{N}\right),
$$

where $\left(\Pi_{*}\right)_{x}: T_{x} \mathcal{M} \rightarrow T_{\Pi(x)} \mathcal{N}$ is the tangent map of $\Pi$ at $x \in \mathcal{M}$. The deterministic Malliavin covariance matrix is defined by

$$
\sigma\left[\psi_{1}\right](h):=D \psi_{1}(h) \circ D \psi_{1}(h)^{*} \in \mathbb{L}\left(T_{\psi_{1}(h)}^{*} \mathcal{N}, T_{\psi_{1}(h)} \mathcal{N}\right),
$$

where the Riesz isometry $\mathcal{H}=\mathcal{H}^{*}$ is implicit.

Similarly, $D X_{t}^{\varepsilon}(w) \in \mathbb{L}\left(\mathcal{H}, T_{X_{t}^{\varepsilon}(w)} \mathcal{M}\right)$ and $D Y_{t}^{\varepsilon}(w)=\left(\Pi_{*}\right)_{X_{t}^{\varepsilon}(w)} \circ D X_{t}^{\varepsilon}(w) \in$ $\mathbb{L}\left(\mathcal{H}, T_{Y_{t}^{\varepsilon}(w)} \mathcal{N}\right)$ for a.a. $w$. The Malliavin covariance matrix of $Y_{1}^{\varepsilon}$ is as follows:

$$
\sigma\left[Y_{1}^{\varepsilon}\right](w):=D Y_{1}^{\varepsilon}(w) \circ D Y_{1}^{\varepsilon}(w)^{*} \in \mathbb{L}\left(T_{Y_{1}^{\varepsilon}(w)}^{*} \mathcal{N}, T_{Y_{1}^{\varepsilon}(w)} \mathcal{N}\right) .
$$

The (deterministic) Malliavin covariance matrix is viewed as a bilinear form on the cotangent space. Since the cotangent space is equipped with an inner product, its eigenvalues and determinant make sense.

Here, we discuss the integration by parts formula for manifold-valued smooth Wiener functionals. This was implicitly used in [48] and is not very difficult. However, since no proof is given in [48], we now give a sketch of proof for the reader's convenience. This formula is used in (i) pullback of a Schwartz distribution on the manifold by a non-degenerate manifold-valued smooth Wiener 
functional, that is a manifold version of Item (c) in Section 3.1 and (ii) upper estimate of the large deviation, that is a manifold version of Theorem 3.8.

Leaving SDE (2.6) aside for a moment, we discuss in a general setting. Let $\mathcal{N}$ be a compact Riemannian manifold and let $F$ be a $\mathbb{D}_{\infty}$-Wiener functional that takes values in $\mathcal{N}$. (For basics of manifold-valued Malliavin calculus, see [48].) The sets of smooth functions and of smooth vector fields on $\mathcal{N}$ are denoted by $C^{\infty}(\mathcal{N})$ and $\Gamma^{\infty}(T \mathcal{N})$, respectively. In a standard way, we identify $T_{y} \mathcal{N}$ and $\mathcal{H}$ with their dual spaces $T_{y}^{*} \mathcal{N}$ and $\mathcal{H}^{*}$, respectively.

Suppose that $D F(w) \circ D F(w)^{*}: T_{F(w)} \mathcal{N} \rightarrow T_{F(w)} \mathcal{N}$ is nonsingular, that is

$$
\operatorname{det}\left[D F(w) \circ D F(w)^{*}\right] \neq 0 \quad \text { for a.a. } w \in \mathcal{W} \text {. }
$$

For $f \in C^{\infty}(\mathcal{N})$ and $Z \in \Gamma^{\infty}(T \mathcal{N})$, substituting

$$
h=D F(w)^{*} \circ\left\{D F(w) \circ D F(w)^{*}\right\}^{-1} Z_{F(w)}
$$

into

$$
D(f(F))(w)\langle h\rangle=\langle D(f(F))(w), h\rangle_{\mathcal{H}}=(\nabla f)(w)\langle D F(w)\langle h\rangle\rangle,
$$

we obtain that

$$
\left\langle D(f(F))(w), D F(w)^{*} \circ\left\{D F(w) \circ D F(w)^{*}\right\}^{-1} Z_{F(w)}\right\rangle_{\mathcal{H}}=(Z f)(F(w)) .
$$

In addition, assume that

$$
(D F)^{*} \circ\left\{D F \circ(D F)^{*}\right\}^{-1} Z_{F} \in \mathbb{D}_{\infty}(\mathcal{H}) \text { for any } Z \in \Gamma^{\infty}(T \mathcal{N}) .
$$

Setting

$$
\begin{gathered}
\Phi_{1}\left(Z^{1} ; G\right)=D^{*}\left[G \cdot\left[(D F)^{*} \circ\left\{(D F) \circ(D F)^{*}\right\}^{-1} Z_{F}^{1}\right]\right], \\
\Phi_{k}\left(Z^{1}, \ldots, Z^{k} ; G\right)=\Phi_{1}\left(Z^{k} ; \Phi_{k-1}\left(Z^{1}, \ldots, Z^{k-1} ; G\right)\right), \quad k=2,3, \ldots
\end{gathered}
$$

for $Z^{1}, \ldots, Z^{k} \in \Gamma^{\infty}(T \mathcal{N})$ and $G \in \mathbb{D}_{\infty}$, and then using equation (8.4) successively, we arrive at the desired integration by parts formula for a manifoldvalued Wiener functional:

$$
\mathbb{E}\left[\left(Z^{1} \cdots Z^{k} f\right)(F) G\right]=\mathbb{E}\left[f(F) \Phi_{k}\left(Z^{1}, \ldots, Z^{k} ; G\right)\right]
$$

for any $Z^{1}, \ldots, Z^{k} \in \Gamma^{\infty}(T \mathcal{N})$ and $G \in \mathbb{D}_{\infty}, f \in C^{\infty}(\mathcal{N})$. For the same reason as in the Euclidean-valued case, this formula extends to the case when $f$ is a Schwartz distribution on $\mathcal{N}$.

The assumptions equation (8.3) and equation (8.5) are satisfied provided that

$$
\begin{gathered}
\left\|\left\{D F \circ(D F)^{*}\right\}^{-1}\right\|_{\mathrm{op}, F} \in \bigcap_{1<p<\infty} L^{p}(\mu) \text { or } \\
\operatorname{det}\left[\left\{D F \circ(D F)^{*}\right\}^{-1}\right] \in \bigcap_{1<p<\infty} L^{p}(\mu) .
\end{gathered}
$$


Here, $\|\cdot\|_{\mathrm{op}, y}$ stands for the operator norm of a linear mapping of $T_{y} \mathcal{N}$ into itself with respect to the Riemannian metric. Since we work on a compact manifold, the above condition is independent of the choice of a Riemannian metric.

By the way, there is another justification of Watanabe's composition $T \circ F$ on manifold. It does not use (8.6), but a localized version of Watanabe's composition theorem in (an open subset of) $\mathbb{R}^{n}$ (not Theorem 3.1 in this paper, but its slightly different variant in Yoshida [53]). Here, we only give a sketch. If a distribution $T$ has a very small support (like the delta functions), then its support is contained in one coordinate chart. Then we can find a suitable real-valued cut-off function on $\mathcal{W}$ and view that $F$, restricted to the support of the cut-off function, takes values in an open subset of $\mathbb{R}^{n}$. Then, we can use the localized version of Watanabe's composition theorem to justify $T \circ F$. Finally, recall that a general distribution on $\mathcal{N}$ can be written as a sum of distributions with small support thanks to a partition of unity on $\mathcal{N}$.

Let us get back to our original setting and assume (B1). For $h \in \mathcal{K}_{a}^{\min }$, the Lagrange multiplier $q(h)=\sigma\left[\psi_{1}\right](h)^{-1} D \psi_{1}(h)\langle h\rangle$ should be regarded as an element of $T_{a}^{*} \mathcal{N}$. Hence, $q(h) \circ\left(\Pi_{*}\right)_{\phi_{1}(h)} \in T_{\phi_{1}(h)}^{*} \mathcal{M}$ and $q(h) \circ\left(\Pi_{*}\right)_{\phi_{1}(h)} \circ J_{1}(h) \in$ $T_{x}^{*} \mathcal{M}$. Recall the latter is the initial value $p_{0}$ of the Hamiltonian ODE associated with Hamiltonian

$$
H(x, p)=\frac{1}{2} \sum_{i=1}^{r}\left\langle p, V_{i}(x)\right\rangle^{2}, \quad(x, p) \in T^{*} \mathcal{M}=\bigcup_{z \in \mathcal{M}} T_{z}^{*} \mathcal{M},
$$

where the pairing is between $T_{x}^{*} \mathcal{M}$ and $T_{x} \mathcal{M}$. Clearly, $H$ is a smooth function on the cotangent bundle $T^{*} \mathcal{M}$.

Then, if Assumption (A1) is replaced by (A1)', Proposition 4.1, Remark 4.2, Proposition 4.3, Proposition 4.4 and Lemma 4.6 also hold in our manifold setting with trivial modifications of the statements. (For instance, $\Pi_{\mathcal{V}}$ should be replaced by $\left(\Pi_{*}\right)_{\phi_{1}(h)}$, and so forth.) A rough sketch of proof is as follows: if we take a local coordinate chart on $\mathcal{M}$, ODEs for $\phi(h)$ and $J(h)$ can be written down in the coordinates. Then, the computation for the Hamiltonian ODE is essentially the same as in Section 4. To prove differentiability or (rough path) continuity of $q(h)$ or $p_{0}=p_{0}(h)$, and so forth in $h$, the extrinsic expression of the skeleton ODE on $\mathbb{R}^{N}$ is better.

As we see in the next lemma, in a neighborhood of the path $t \mapsto \phi_{t}(h)$, $h \in \mathcal{K}_{a}^{\text {min }}$, the RDE on $\mathcal{M}$ can be transferred to an RDE on $\mathbb{R}^{d}$. Hence, for local analysis around $h$ of the RDE on manifold, it is sufficient to deal with the corresponding RDE on the Euclidean space. This is one of main advantages of using rough path theory since in the classical theory of Itô maps this kind of 'local' operation is not easy and sometimes impossible. 
LEMMA 8.1. (i) For any $h \in \mathcal{K}_{a}^{\min }$, we can find the following (1)-(4):

(1) An open neighborhood $\mathcal{U}=\mathcal{U}_{h}$ of $\operatorname{Im} \phi(h):=\left\{\phi_{t}(h) \mid t \in[0,1]\right\}$ in $\mathcal{M}$.

(2) A bounded, connected, open subset $\mathcal{U}^{\prime}=\mathcal{U}_{h}^{\prime}$ in $\mathbb{R}^{d}$.

(3) A diffeomorphism $\theta=\theta_{h}: \mathcal{U} \rightarrow \mathcal{U}^{\prime}$.

(4) Vector fields $V_{i}^{(h)}$ on $\mathbb{R}^{d}$ of class $C_{b}^{\infty}$ such that $\left(\theta_{*}\right)_{x} V_{i}(x)=V_{i}^{(h)}(\theta(x))$ $(0 \leqslant i \leqslant r)$.

(ii) Consider skeleton $O D E$ (2.2) and $R D E$ (3.17) on $\mathbb{R}^{d}$, but with the coefficients $V_{i}^{(h)}(0 \leqslant i \leqslant r)$ and the initial point $\theta(x)$. If $k \in \mathcal{H}$ is sufficiently close to $h$ in $\mathcal{H}$-topology, then $t \mapsto \theta\left(\phi_{t}(k)\right)$ solves the skeleton $O D E$ on $\mathbb{R}^{d}$ driven by $k$. Likewise, if $\mathbf{w}$ and $\mathbf{h}=\mathcal{L}(h)$ are sufficiently close in $G \Omega_{\alpha, 4 m}^{B}\left(\mathbb{R}^{d}\right)$ and $\|\lambda\|_{1-H}$ is sufficiently small, then $t \mapsto \theta\left(x+\mathbf{x}_{0, t}^{1}\right)=\theta\left(x+\Phi(\mathbf{w}, \lambda)_{0, t}^{1}\right)$ is the first level path of the solution of the $R D E$ on $\mathbb{R}^{d}$ driven by $(\mathbf{w}, \lambda)$.

Proof. First, we prove (i). Clearly, the image of the path $\phi(h)$ is compact in $\mathcal{M}$ and there is no selfintersection due to the energy-minimizing condition. Moreover, by Remark 4.2, $\phi_{t}^{\prime}(h)$ never vanishes. Therefore, we can use the standard technique of the tubular neighborhood to prove such $\mathcal{U}, \mathcal{U}^{\prime}, \theta$ exist. Then, existence of such $V_{i}^{(h)}$ is almost obvious. Since the (Lyons-)Itô maps associated with the skeleton ODE and the RDE are continuous, the assertion (ii) is easy.

REMARK 8.2. To keep our notations simple, we often identify $\mathcal{U}$ and $\mathcal{U}^{\prime}$ through $\theta$. In such cases we compute ODE/RDE/SDE on $\mathbb{R}^{d}$ with the coefficients $V_{i}^{(h)}$ and the initial point $x \in \mathbb{R}^{d}$. The solution of SDE (2.1) associated with $V_{i}^{(h)}$ is denoted by $X^{\varepsilon,(h)}$. (This should not be confused with $X_{t}^{\varepsilon, h}:=X^{\varepsilon}(t, x, w+h / \varepsilon)$.) Likewise, $\phi(k), \Phi(\mathbf{w}, \lambda)$ associated with $V_{i}^{(h)}$ are denoted by $\phi^{(h)}(k), \Phi^{(h)}(\mathbf{w}$, $\lambda)$, respectively. The ordinary terms and the remainder term in the expansion of $X^{\varepsilon,(h)}(t, x, w+k / \varepsilon)$ in (3.29) and (3.32) are denoted by $f_{j}^{(h)}(w ; k), Q_{j+1}^{\varepsilon,(h)}(w ; k)$ and $\hat{f}_{j}^{(h)}(\mathbf{w} ; k), \hat{Q}_{j+1}^{\varepsilon,(h)}(\mathbf{w} ; k)$, respectively.

Next, we take the normal coordinate around $a \in \mathcal{N}$. Then, there exists a diffeomorphism from a neighborhood of $a$ to a neighborhood of 0 in $T_{a} \mathcal{N}$. Note that $T_{a} \mathcal{N}$ is a vector space with an inner product. Through this diffeomorphism, the delta function at $a \in \mathcal{N}$ corresponds to the delta function at $0 \in T_{a} \mathcal{N}$. Moreover, since the tangent map of this diffeomorphism at $a$ is isometric and so is its transpose, the determinant and the eigenvalues of the deterministic Malliavin covariance matrix of $\phi_{1}(h)$ for $h \in \mathcal{K}_{a}$ remains the same even if they are calculated in the normal coordinate. 
Let $h \in \mathcal{K}_{a}^{\min }$ and let $\mathcal{U}, \mathcal{U}^{\prime}$ and $\theta$ be as in Lemma 8.1. (They all depend on $h$.) If we take a small neighborhood of $\theta\left(\phi_{1}(h)\right) \in \mathcal{U}^{\prime}$, then $\Pi$ (viewed as a map from $\mathcal{U}^{\prime}$ ) maps it into the normal coordinate of $a \in \mathcal{N}$. In a standard way we can find a $C_{b}^{\infty}$-map $\Pi^{(h)}$ from $\mathbb{R}^{d}$ to $T_{a} \mathcal{N}$ which agrees with $\Pi$ when restricted to the neighborhood of $\theta\left(\phi_{1}(h)\right)$. Consequently, for $\varepsilon(h)>0$ and $\gamma(h)>0$ small enough, it holds that

$$
\begin{aligned}
& \Pi^{(h)}\left[x+\Phi^{(h)}\left(\varepsilon \mathbf{W}, \lambda^{\varepsilon}\right)_{0,1}^{1}\right]=Y^{\varepsilon}(1, x, w) \in T_{a} \mathcal{N} \\
& \quad \text { if } \varepsilon \in[0, \varepsilon(h)] \text { and } \varepsilon \mathbf{W} \in U_{h, \gamma(h)} .
\end{aligned}
$$

(Precisely, this means that $Y^{\varepsilon}(t, x, w)$ takes values in the normal coordinate of $a$ if $\varepsilon \in[0, \varepsilon(h)]$ and $\varepsilon \mathbf{W} \in U_{h, \gamma(h)}$ and the value is equal to the left hand side.)

Asymptotic expansion of

$$
\Pi^{(h)}\left[x+\Phi^{(h)}\left(\tau_{k}(\varepsilon \mathbf{W}), \lambda^{\varepsilon}\right)_{0,1}^{1}\right]=\Pi^{(h)}\left[X^{\varepsilon,(h)}\left(1, x, w+\frac{k}{\varepsilon}\right)\right]
$$

was already done in Lemma 3.6. We write $g_{j}^{(h)}(w ; k)$ and $R_{j+1}^{\varepsilon,(h)}(w ; k)$ for $g_{j}^{F}(w ; k)$ and $R_{j+1}^{\varepsilon, F}(w ; k)$ in Lemma 3.6, respectively (with $\left.F=\Pi^{(h)}\right)$. Thus, local analysis of the solution of RDE on manifold $\mathcal{M}$ was reduced to that in the linear setting.

REMARK 8.3. The definition of $\mathcal{K}_{a}^{\mathrm{min}}$ depends on the coefficient vector fields of the skeleton ODE. If they are replaced by new vector fields $V_{i}^{(h)}(0 \leqslant i \leqslant r), \mathcal{K}_{a}^{\text {min }}$ may change and $k \in \mathcal{K}_{a}^{\text {min }}$ that is close enough to $h$ may not belong to $\mathcal{K}_{a}^{\min ,(h)}$ anymore. However, we need not worry about this for the following reasons: such $k$ is still a local minimum of $k \mapsto\|k\|_{\mathcal{H}}^{2} / 2$ subject to $\psi_{1}^{(h)}(k)=\Pi^{(h)}\left(\phi_{1}^{(h)}(k)\right)=$ $0 \in T_{a} \mathcal{N}$ and all the computations for the skeleton ODE, but one, require just the local minimum property via the Lagrange multiplier method (So $k$ need not be a global minimizer of this conditional minimal problem in the proof of the asymptotic expansion.) The only exception where the global minimum property is really used is the proof of the large deviation upper bound, in which we compute original $\psi_{1}$ and $\mathcal{K}_{a}^{\min }$, not $\psi_{1}^{(h)}$ and $\mathcal{K}_{a}^{\min ,(h)}$.

Now we compute the second order terms. Let $h \in \mathcal{K}_{a}^{\text {min }}$ and take $\mathcal{U}$ and $V_{i}^{(h)}(0 \leqslant i \leqslant r)$ as in Lemma 8.1. Suppose that $k$ is sufficiently close to $h$. The Lagrange multiplier $q(k) \in T_{a}^{*} \mathcal{N}$, which is independent of $h$, is now an $n$ dimensional row vector and (4.3) holds with $\mathcal{V}$ replaced by $\mathbb{R}^{n} \cong T_{a} \mathcal{N}$.

We can compute $D \phi_{t}^{(h)}(k)\langle l\rangle$ and $D^{2} \phi_{t}^{(h)}(k)\langle l, \hat{l}\rangle$ in the same way as in (4.1) and (4.2), respectively (if $V_{i}$ are replaced by $V_{i}^{(h)}$ ).

Since $k$ and $h$ are close, $\Pi\left(\phi_{1}(k)\right)=\Pi^{(h)}\left(\phi_{1}^{(h)}(k)\right)$ and we do not distinguish them in this paragraph. We denote by $\nabla$ the usual gradient for maps from an open 
subset of $\mathbb{R}^{d}$ to $T_{a} \mathcal{N} \cong \mathbb{R}^{n}$. Then, we have

$D \psi_{1}^{(h)}(k)\langle l\rangle=(\nabla \Pi)\left(\phi_{1}^{(h)}(k)\right)\left\langle D_{l} \phi_{1}^{(h)}(k)\right\rangle=\left(\Pi_{*}\right)_{y} D_{l} \phi_{1}^{(h)}(k) \quad\left(\right.$ with $\left.y=\phi_{1}^{(h)}(k)\right)$

and

$$
\begin{aligned}
D^{2} \psi_{1}^{(h)}(k)\left\langle l, l^{\prime}\right\rangle= & (\nabla \Pi)\left(\phi_{1}^{(h)}(k)\right)\left\langle D_{l, l^{\prime}}^{2} \phi_{1}^{(h)}(k)\right\rangle \\
& +\left(\nabla^{2} \Pi\right)\left(\phi_{1}^{(h)}(k)\right)\left\langle D_{l} \phi_{1}^{(h)}(k), D_{l^{\prime}} \phi_{1}^{(h)}(k)\right\rangle .
\end{aligned}
$$

Here, we wrote $D_{l} \phi_{1}^{(h)}(k)=D \phi_{1}^{(h)}(k)\langle l\rangle$ and $D_{l, l^{\prime}}^{2} \phi_{1}^{(h)}(k)=D^{2} \phi_{1}^{(h)}(k)\left\langle l, l^{\prime}\right\rangle$ for simplicity.

Note that the same computations as in (5.1) and (5.2) still hold if $k$ is close to $h$. Hence, if $O_{h}$ is a sufficiently small neighborhood of $h$ in $\mathcal{K}_{a}^{\min }$, then $\sup \left\{C^{(h)}(k) \mid\right.$ $\left.k \in O_{h}\right\}<1 / 2$ instead of (5.4).

For the same reason as before, if $k$ is sufficiently close to $h$,

$$
\left\langle q(k), g_{2}^{(h)}\left(\pi^{k} w ; k\right)\right\rangle-\mathbb{E}\left[\left\langle q(k), g_{2}^{(h)}\left(\pi^{k} w ; k\right)\right\rangle\right]
$$

belongs to $\mathcal{C}_{2}$, the second order homogeneous Wiener chaos, and corresponds to the Hilbert-Schmidt bilinear form

$$
\frac{1}{2}\left\langle q(k), D^{2} \psi_{1}^{(h)}(k)\left\langle\pi^{k} \bullet, \pi^{k} \star\right\rangle\right\rangle .
$$

To check the correspondence, just apply $(1 / 2) D^{2}$ to $g_{2}^{F}\left(\pi^{k} w ; k\right)$ with $F=\Pi^{(h)}$ in Lemma 3.6(i) and compare it with (8.7).

From this and $\sup \left\{C^{(h)}(k) \mid k \in O_{h}\right\}<1 / 2$, we have the following under (A1)' and (B1)-(B3): for any $h \in \mathcal{K}_{a}^{\text {min }}$, there exits $c_{1}=c_{1}(h)>1$ and a neighborhood $O_{h}$ of $h$ in $\mathcal{K}_{a}^{\min }$ such that

$$
\sup _{k \in O_{h}} \mathbb{E}\left[\exp \left(c_{1}\left\langle q(k), g_{2}^{(h)}(w ; k)\right\rangle\right) \delta_{0}\left(g_{1}^{(h)}(w ; k), \mathbf{i}_{k}\langle w\rangle\right)\right]<\infty .
$$

Here, $\delta_{0}$ is the Dirac delta function on $\mathbb{R}^{n} \times T_{k} \mathcal{K}_{a}^{\text {min }}$. (The proof is essentially the same as in Lemma 5.3.) Moreover, from (8.8) we obtain the following: for any $c_{2} \in\left(1, c_{1}(h)\right)$, there exists a constant $\gamma_{1}=\gamma_{1}(h)>0$ which is independent of $k \in O_{h}$ and satisfies that

$$
\begin{gathered}
\sup _{0<\varepsilon \leqslant 1} \sup _{k \in O_{h}} \mathbb{E}\left[\exp \left(c_{2}\left\langle q(k), \frac{R_{2}^{\varepsilon,(h)}(w ; k)}{\varepsilon^{2}}\right\rangle\right) \mathbf{1}_{U_{\gamma_{1}}}(\varepsilon \mathbf{W}) ; A(h, k, \varepsilon, \kappa)\right]<\infty \\
\text { where } A(h, k, \varepsilon, \kappa)=\left\{\left|\mathbf{i}_{k}\right|^{2}+\left|\frac{R_{1}^{\varepsilon,(h)}(\cdot ; k)}{\varepsilon}\right|^{2} \leqslant \kappa^{2}\right\}
\end{gathered}
$$

for any $\kappa>0$. (The proof is essentially the same as in Lemma 5.3.) 
In a similar way, Lemma 3.7 can be modified. If $O_{h}$ is sufficiently small, then

$$
\lambda(h):=\inf _{k \in O_{h}} \inf _{z:|z|=1} z^{*} \sigma\left[\psi_{1}^{(h)}\right](k) z>0 .
$$

Taking $O_{h}$ smaller if necessary, we have the following: there exist $\gamma_{0}=\gamma_{0}(h)$, $\varepsilon_{0}=\varepsilon_{0}(h) \in(0,1]$ such that the smallest eigenvalue of $\sigma\left[\left(R_{1}^{\varepsilon,(h)}(\cdot ; k) / \varepsilon, \mathbf{i}_{k}\right)\right](w)$ is greater than $(\lambda(h) \wedge 1) / 2$ if $\varepsilon \mathbf{W} \in U_{\gamma_{0}}, k \in O_{h}$ and $0<\varepsilon \leqslant \varepsilon_{0}$. (Here, $\psi_{1}^{(h)}$ and $R_{1}^{\varepsilon,(h)}(\cdot ; k) / \varepsilon$ are regarded as $T_{a}^{*} \mathcal{N} \cong \mathbb{R}^{n}$-valued maps.)

In the same way as in Lemma 6.2, we have the following lemma. Note that in this case the constants $\lambda, \gamma_{0}, \gamma_{1}, \varepsilon_{0}$ all depend on $h \in \mathcal{K}_{a}^{\text {min }}$ (which makes no difference, however). The definition of $\sigma=\sigma_{h}$ is in (6.7).

LEMMA 8.4. For any $h \in \mathcal{K}_{a}^{\min }$, we can find $O_{h}$ (or $\rho=\rho_{h}:=\sup _{k \in O_{h}}\|k-h\|_{\mathcal{H}}>$ $0), \gamma=\gamma_{h}>0$ and $\varepsilon_{0}=\varepsilon_{0}(h) \in(0,1]$ such that (6.4), (6.5), (6.6) and the following three conditions hold. The first one is:

$$
\begin{aligned}
& \inf _{k \in O_{h}} \inf _{z:\|z\|=1} z^{*} \sigma\left[\left(R_{1}^{\varepsilon,(h)}(\cdot ; k) / \varepsilon, \mathbf{i}_{k}\right)\right](w) z \geqslant \frac{\lambda(h) \wedge 1}{2} \\
& \quad \text { if } \varepsilon \in\left(0, \varepsilon_{0}\right] \text { and } \varepsilon \mathbf{W} \in U_{h-k, 2^{1 / 4 m} \sigma \gamma} .
\end{aligned}
$$

The second one is:

$$
\begin{aligned}
& \sup _{0<\varepsilon \leqslant 1} \sup _{k \in O_{h}} \mathbb{E}\left[\exp \left(\frac{c_{2}}{\varepsilon^{2}}\left\langle q(k), R_{2}^{\varepsilon,(h)}(w ; k)\right\rangle\right) \mathbf{1}_{U_{h-k, \gamma}}(\varepsilon \mathbf{W}) ;\left|\mathbf{i}_{k}\right|^{2}\right. \\
& \left.+\left|\frac{R_{1}^{\varepsilon,(h)}(w ; k)}{\varepsilon}\right|^{2} \leqslant \kappa^{2}\right]<\infty .
\end{aligned}
$$

The last one is:

$$
\begin{aligned}
& \left\{x+\Phi\left(\mathbf{w}, \lambda^{\varepsilon}\right)_{0, t}^{1} \mid t \in[0,1], \varepsilon \in\left[0, \varepsilon_{0}\right], \mathbf{w} \in U_{h, \gamma}\right\} \\
& \quad \cup\left\{\phi(k)_{t} \mid t \in[0,1], k \in O_{h}\right\} \\
& \quad \subset \mathcal{U}_{h} \subset \mathcal{M},
\end{aligned}
$$

where $\mathcal{U}_{h} \subset \mathcal{M}$ is the tubular neighborhood of $\operatorname{Im} \phi(h)$ introduced in Lemma 8.1.

Proof. The proof is the same as in Lemma 6.2.

In what follows let $\gamma_{h}$ be the constant in Lemma 6.2. Since $\gamma_{h}>0$ for all $h \in \mathcal{K}_{a}^{\min }$, we can choose finitely many $\left(h_{1}, \gamma_{1}\right), \ldots,\left(h_{N}, \gamma_{N}\right)$ and construct asymptotic partition of unity $\chi^{\varepsilon}$ and $\tilde{\chi}_{v}^{\varepsilon}$ exactly in the same way as 
in (6.1)-(6.2). (Here, $\gamma_{v}$ stands for $\gamma_{h}$ with $h=h_{\nu}$.) Note that the definitions of $\chi^{\varepsilon}$ and $\tilde{\chi}_{v}^{\varepsilon}$ depend only on $\left(h_{v}, \gamma_{v}\right)_{1 \leqslant v \leqslant N}$. Below we set $\varepsilon_{0}^{\prime}:=\min _{1 \leqslant v \leqslant N} \varepsilon_{0}\left(h_{v}\right)$ $\in(0,1]$ and $\lambda^{\prime}:=\min _{1 \leqslant v \leqslant N} \lambda\left(h_{v}\right)>0$.

As in the Euclidean case, contribution of the rough paths away from $\mathcal{K}_{a}^{\text {min }}$ is negligible. To prove it, we need to modify Theorem 3.8, the large deviation upper estimate, to the manifold case. (Once this is done, a manifold version of Theorem 7.1 can be obtained in the same way.)

The keys of the proof of Theorem 3.8 are Kusuoka-Stroock's moment estimate (2.4) of $\operatorname{det} \sigma\left[Y_{1}^{\varepsilon}\right]^{-1}$ and the integration by parts formula for Watanabe distributions. Both of them hold in the manifold case (see (8.6)). Therefore, we can prove Theorem 3.8 in the manifold case, too. Consequently, $I_{1}(\varepsilon)$ does not contribute to the asymptotic expansion.

Proof of Theorem 2.5. It is sufficient to compute

$$
I_{2}(\varepsilon)=\sum_{\nu=1}^{N} \mathbb{E}\left[\tilde{\chi}_{\nu}^{\varepsilon} G^{\varepsilon} \delta_{a}\left(Y_{1}^{\varepsilon}\right)\right]
$$

as $\varepsilon \in\left(0, \varepsilon_{0}^{\prime}\right]$ tends to zero. Here, $\delta_{a}$ is the Dirac measure at $a \in \mathcal{N}$ and $Y_{1}^{\varepsilon}=$ $\Pi\left(X_{1}^{\varepsilon}\right)$ with $\left(X_{t}^{\varepsilon}\right)$ being the solution of the $\mathcal{M}$-valued SDE (2.6).

Let $\eta \in C_{0}^{\infty}\left(T_{a} \mathcal{N}, \mathbb{R}\right)$ be as in (7.2) in Section 7. Then, defined by $\eta_{l}(y)=$ $l^{n} \eta(l y),\left\{\eta_{l}\right\}_{l \geqslant 1}$ approximates $\delta_{0}$, where $\delta_{0}$ is the delta function at 0 on $T_{a} \mathcal{N} \cong$ $\mathbb{R}^{n}$ with respect to the Lebesgue measure associated with the inner product on $T_{a} \mathcal{N}$. Denote by $\hat{\eta}_{l} \in C_{0}^{\infty}(\mathcal{N}, \mathbb{R})$ the function which corresponds to $\eta_{l}$ via the exponential map at $a$. Then, $\left\{\hat{\eta}_{l}\right\}_{l \geqslant 1}$ approximates the delta function at $a$ on $\mathcal{N}$. Therefore,

$$
\mathbb{E}\left[\tilde{\chi}_{\nu}^{\varepsilon} G^{\varepsilon} \delta_{a}\left(Y_{1}^{\varepsilon}\right)\right]=\lim _{l \rightarrow \infty} \mathbb{E}\left[\tilde{\chi}_{\nu}^{\varepsilon} G^{\varepsilon} \hat{\eta}_{l}\left(Y_{1}^{\varepsilon}\right)\right]=\lim _{l \rightarrow \infty} \mathbb{E}\left[\tilde{\chi}_{\nu}^{\varepsilon} G^{\varepsilon} \eta_{l}\left(Y_{1}^{\varepsilon,(\nu)}\right)\right]
$$

since $\tilde{\chi}_{v}^{\varepsilon}=0$ if $\varepsilon \mathbf{W} \notin U_{h_{v}, \gamma_{v}}$. Here $Y_{1}^{\varepsilon,(v)}$ is a shorthand for

$$
Y_{1}^{\varepsilon,(h)}=\Pi^{(h)}\left[X^{\varepsilon,(h)}(1, x, w)\right] \quad \text { with } h=h_{v} .
$$

Note that the right hand side of (8.11) is essentially the same as the left hand side of (7.2). Thus, the asymptotic problem has been reduced to the one on the Euclidean space. Note also that we need not prove non-degeneracy of $\sigma\left[Y_{1}^{\varepsilon,(h)}\right]$ because we use Propositions 3.1 and 3.2.

Define $\mathcal{E}_{j}^{(v)}(w ; k) \in \tilde{\mathbb{D}}_{-\infty}$ in the same way as in (7.14). (This time it depends on $v$ (or $h_{v}$ ), however.) By the same computation as in (7.16), we have the following 
asymptotics: for any $L \in \mathbb{N}$,

$$
\begin{aligned}
\mathbb{E}\left[\tilde{\chi}_{v}^{\varepsilon} G^{\varepsilon} \delta_{a}\left(Y_{1}^{\varepsilon}\right)\right]= & \int_{O_{v}} \mathbb{E}\left[\exp \left(\left\langle q(k), g_{2}^{(v)}(w ; k)\right\rangle\right)\right. \\
& \left.\times\left(\sum_{j=0}^{L} \varepsilon^{j} \mathcal{E}_{j}^{(v)}(w ; k)+O\left(\varepsilon^{L+1}\right)\right) \tilde{\chi}_{v}^{\varepsilon}\left(w+\frac{k}{\varepsilon}\right)\right] \omega(d k) \\
= & \int_{O_{v}} \mathbb{E}\left[\exp \left(\left\langle q(k), g_{2}^{(v)}(w ; k)\right\rangle\right)\left(\sum_{j=0}^{L} \varepsilon^{j} \mathcal{E}_{j}^{(v)}(w ; k)\right)\right. \\
& \left.\times\left(\sum_{l=0}^{L} \varepsilon^{l} \mathcal{D}_{l}^{(v)}(w ; k)\right)\right] \omega(d k) \\
& +O\left(\varepsilon^{L+1}\right)
\end{aligned}
$$

as $\varepsilon \searrow 0$. Note that the asymptotic expansion inside $\int_{O_{v}} \omega(d k)$ is uniform in $k \in O_{v}$. Vanishing of $c_{2 j+1}$ under (C2) can be shown in the same way as in the Euclidean case. This completes the proof of Theorem 2.5.

\section{Acknowledgements}

The first-named author is partially supported by JSPS KAKENHI Grant Number 15K04922, and the second-named author is partially supported by JSPS KAKENHI Grant Number $15 \mathrm{~K} 04931$.

\section{References}

[1] S. Aida, 'Vanishing of one-dimensional $L^{2}$-cohomologies of loop groups', J. Funct. Anal. 261 (2011), 2164-2213.

[2] I. Bailleul, 'Flows driven by rough paths', Rev. Mat. Iberoam. 31 (2015), 901-934.

[3] D. Barilari, U. Boscain and R. Neel, 'Small-time heat kernel asymptotics at the subRiemannian cut locus', J. Differential Geom. 92 (2012), 373-416.

[4] D. Barilari and J. Jendrej, 'Small time heat kernel asymptotics at the cut locus on surfaces of revolution', Ann. Inst. H. Poincaré Anal. Non Linéaire 31 (2014), 281-295.

[5] F. Baudoin and J. Wang, 'The subelliptic heat kernel on the CR sphere', Math. Z. 275 (2013), $135-150$.

[6] R. Beals, B. Gaveau and P. C. Greiner, 'Hamilton-Jacobi theory and the heat kernel on Heisenberg groups', J. Math. Pures Appl. 79 (2000), 633-689.

[7] G. Ben Arous, 'Développement asymptotique du noyau de la chaleur hypoelliptique hors du cut-locus', Ann. Sci. Éc. Norm. Supér. 21 (1988), 307-331.

[8] J.-M. Bismut, Large Deviations and the Malliavin Calculus, Progress in Mathematics, 45 (Birkhäuser Boston, Inc., Boston, MA, 1984).

[9] V. Bogachev, Gaussian Measures (American Mathematical Society, Providence, RI, 1998). 
[10] R. W. Brockett and A. Mansouri, 'Short-time asymptotics of heat kernels for a class of hypoelliptic operators', Amer. J. Math. 131 (2009), 1795-1814.

[11] O. Calin and D.-C. Chang, 'Sub-Riemannian geometry', in General Theory and Examples (Cambridge University Press, Cambridge, 2009).

[12] D.-C. Chang and Y. Li, 'Heat kernel asymptotic expansions for the Heisenberg sub-Laplacian and the Grushin operator', Proc. A. 471(2175) (2015), 20140943, 19 pp.

[13] D.-C. Chang, I. Markina and A. Vasil'ev, 'Hopf fibration: geodesics and distances', J. Geom. Phys. 61(6) (2011), 986-1000.

[14] J. D. Deuschel, P. K. Friz, A. Jacquier and S. Violante, 'Marginal density expansions for diffusions and stochastic volatility I: theoretical foundations', Comm. Pure Appl. Math. 67 (2014), 40-82.

[15] S. Dragomir and G. Tomassini, Differential Geometry and Analysis on CR Manifolds (Birkhäuser Boston, Inc., Boston, MA, 2006).

[16] P. Friz and N. Victoir, Multidimensional Stochastic Processes as Rough Paths (Cambridge University Press, Cambridge, 2010).

[17] B. Gaveau, 'Principe de moindre action, propagation de la chaleur et estimées sous elliptiques sur certains groupes nilpotents', Acta Math. 139 (1977), 95-153.

[18] E. Grong and A. Thalmaier, 'Curvature-dimension inequalities on sub-Riemannian manifolds obtained from Riemannian foliations, Part I', Math. Z. 282 (2016), 99-130.

[19] E. P. Hsu, Stochastic Analysis on Manifolds (American Mathematical Society, Providence, RI, 2002).

[20] Y. Hu, Analysis on Gaussian Spaces (World Scientific, Singapore, 2017).

[21] N. Ikeda, 'Probabilistic methods in the study of asymptotics', in École d'été de Probabilistés de Saint-Flour XVIII, 1988, Lecture Notes in Mathematics, 1427 (Springer, Berlin, 1990), 195-325.

[22] N. Ikeda and S. Watanabe, Stochastic Differential Equations and Diffusion Processes, 2nd edn (North-Holland Publishing Co., Amsterdam; Kodansha, Ltd., Tokyo, 1989).

[23] Y. Inahama, 'Quasi-sure existence of Brownian rough paths and a construction of Brownian pants', Infin. Dimens. Anal. Quantum Probab. Relat. Top. 9 (2006), 513-528.

[24] Y. Inahama, 'A stochastic Taylor-like expansion in the rough path theory', J. Theoret. Probab. 23 (2010), 671-714.

[25] Y. Inahama, 'Large deviation principle of Freidlin-Wentzell type for pinned diffusion processes', Trans. Amer. Math. Soc. 367 (2015), 8107-8137.

[26] Y. Inahama, 'Short time kernel asymptotics for rough differential equation driven by fractional Brownian motion', Electron. J. Probab. 21 (2016), Paper No. 34, 29 pp.

[27] Y. Inahama, 'Large deviations for rough path lifts of Watanabe's pullbacks of delta functions', Int. Math. Res. Not. IMRN 2016 (2016), 6378-6414.

[28] Y. Inahama and K. Kawabi, 'Asymptotic expansions for the Laplace approximations for Itô functionals of Brownian rough paths', J. Funct. Anal. 243 (2007), 270-322.

[29] H. Kondo and S. Taniguchi, 'A construction of diffusion processes associated with subLaplacian on CR manifolds and its applications', J. Math. Soc. Japan 69 (2017), 111-125.

[30] S. Kusuoka and D. W. Stroock, 'Applications of the Malliavin calculus. II', J. Fac. Sci. Univ. Tokyo Sect. IA Math. 32 (1985), 1-76.

[31] S. Kusuoka and D. W. Stroock, 'Precise asymptotics of certain Wiener functionals', J. Funct. Anal. 99 (1991), 1-74.

[32] S. Kusuoka and D. W. Stroock, 'Asymptotics of certain Wiener functionals with degenerate extrema’, Comm. Pure Appl. Math. 47 (1994), 477-501. 
[33] M. Ludewig, 'Strong short time asymptotics and convolution approximation of the heat kernel', Preprint 2016, arXiv:1607.05152.

[34] T. Lyons, M. Caruana and T. Lévy, Differential Equations Driven by Rough Paths, Lecture Notes in Mathematics, 1908 (Springer, Berlin, 2007).

[35] T. Lyons and Z. Qian, System Control and Rough Paths (Oxford University Press, Oxford, 2002).

[36] P. Malliavin, Stochastic Analysis (Springer, Berlin, 1997).

[37] H. Matsumoto and S. Taniguchi, Stochastic Analysis: Itô and Malliavin Calculus in Tandem (Cambridge University Press, Cambridge, 2017).

[38] S. A. Molchanov, 'Diffusion processes, and Riemannian geometry', Russian Math. Surveys 30 (1975), 1-63.

[39] M. Molina and I. Markina, 'Sub-Riemannian geodesics and heat operator on odd dimensional spheres', Anal. Math. Phys. 2 (2012), 123-147.

[40] R. Montgomery, A Tour of Subriemannian Geometries, their Geodesics and Applications, (American Mathematical Society, Providence, RI, 2002).

[41] D. Nualart, The Malliavin Calculus and Related Topics, 2nd edn (Springer, Berlin, 2006).

[42] L. Rifford, Sub-Riemannian Geometry and Optimal Transport, Springer Briefs in Mathematics (Springer, Cham, 2014).

[43] C. Séguin and A. Mansouri, 'Short-time asymptotics of heat kernels of hypoelliptic Laplacians on unimodular Lie groups', J. Funct. Anal. 262 (2012), 3891-3928.

[44] I. Shigekawa, 'Stochastic analysis', in Translations of Mathematical Monographs, Vol. 224, Iwanami Series in Modern Mathematics (American Mathematical Society, Providence, RI, 2004).

[45] D. W. Stroock, An Introduction to the Analysis of Paths on a Riemannian Manifold (American Mathematical Society, Providence, 2000).

[46] H. Sugita, 'Positive generalized Wiener functions and potential theory over abstract Wiener spaces', Osaka J. Math. 25 (1988), 665-696.

[47] S. Takanobu and S. Watanabe, 'Asymptotic expansion formulas of the Schilder type for a class of conditional Wiener functional integrations', in Asymptotic Problems in Probability Theory: Wiener Functionals and Asymptotics (Sanda/Kyoto, 1990), Pitman Research Notes in Mathematics Series, 284 (Longman Sci. Tech., Harlow, 1993), 194-241.

[48] S. Taniguchi, 'Malliavin's stochastic calculus of variations for manifold-valued Wiener functionals and its applications', Z. Wahrsch. Verw. Gebiete 65 (1983), 269-290.

[49] H. Uemura, 'Off-diagonal short time expansion of the heat kernel on a certain nilpotent Lie group', Kyoto J. Math. 30 (1990), 403-449.

[50] H. Uemura and S. Watanabe, 'Diffusion processes and heat kernels on certain nilpotent groups', in Stochastic Analysis (Paris, 1987), Lecture Notes in Mathematics, 1322 (Springer, Berlin, 1988), 173-197.

[51] S. Watanabe, Lectures on Stochastic Differential Equations and Malliavin Calculus (Tata Institute of Fundamental Research, Bombay, Springer, Berlin, 1984).

[52] S. Watanabe, 'Analysis of Wiener functionals (Malliavin calculus) and its applications to heat kernels', Ann. Probab. 15 (1987), 1-39.

[53] N. Yoshida, 'Asymptotic expansions of maximum likelihood estimators for small diffusions via the theory of Malliavin-Watanabe', Probab. Theory Related Fields 92 (1992), 275-311. 